\title{
Docanmin
}

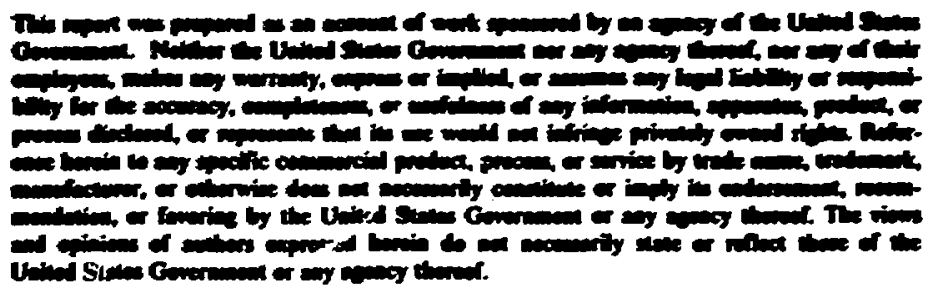

UCRL- $-50200-\mathrm{V} 01.25$

DECA 007014

\section{Omega Documentation}

\author{
R. J. Howerton, R. E. Dye, P. C. Giles, \\ J. R. Kimlinger, S. T. Perkins, and E. F. Plechaty
}

Manuscript date: August 1983

\section{LAWRENCE LIVERMORE NATIONAL LABORATORY University of California - Livermore, California - 94550}




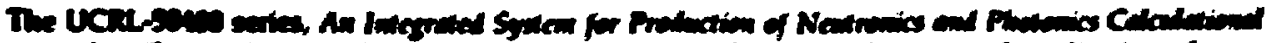

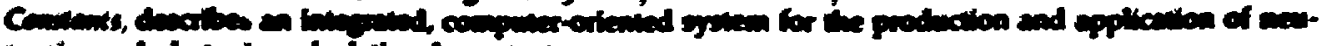

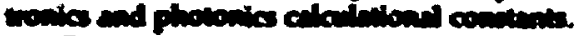

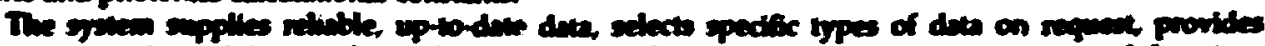

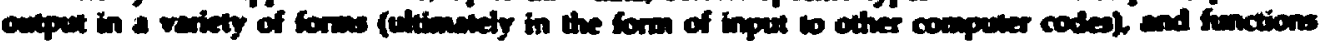
apidity and efixitendy.

The UCRL-SOHD series comprises the following rolumes:

- Vol. 1, Pat A, Rev. 3, ECSIL: a Sydem for Storage, Retriedal, and Diplay of Experimental Newtrom Duta, Septesuber 1976.

- Vol. 1. Part B, Progrem ECSX\$ (Version 78-1): Conoersion of Experiment 7lly Measured Cross-Section

Data from the Four-Center-Exchange $(X-4)$ Format to the Livermore ECSIL Formal December 1978.

- Vol. 2, Rev. 2, A Batiography of the Experimental Date of Neutron-Induceri Interactions, July 1976.

- Vol. 3, Rev. 2, An Index of the Experimental Data of Neutron-Jnduced Interactions, July 1976.

- Vol. 4, Rev. 1, Eoaluated Nuclear Data Lbrary. September 1981.

- Vol. 4, Rev. 1, Appendix C, The Neulron Lbrary (ENDL82) in the Transmitt.al Formet, June 1982.

- Vol. 5, Part A, Rev. 1, CLYDE: A Code for the Production of Calculational Constants from Nuclear Data, September 1975.

- Vol. 5, Part B, Rev. 1, Relativistic Transformations between Center-of-Mass and Laboratory Systems for Tizo-Body Nuclear Reactions, April 1978.

- Vol. 6, Rev. 2, Tables and Graphs of Photon-Interaction Cross Sections from $1 \mathrm{keV} \pm 0100 \mathrm{MeV}$, December 1978 .

- Vol. 7, Part A, Rev. 1, Major Neutron-Induced Interactions ( $Z \leq 55)$ : Graphical Experimental Data, July 1976.

- Vol. 7, Part B, Rev. 1, Major Neutron-Induced Interactions ( $>$ > 55): Graphical, Experimental Data, July 1976.

- Vol. 8, Part A, Rev. 1, Supplemental Neutron-Induced Interactions ( $Z \leq 35)$ : Graphical, Experimental Data, July 1976.

- Vol. 8, Part B, Rev. 1, Supplemental Neutron-Induced Interactions $(Z>35)$ : Graphical, Experimental Data, July 1976.

- Vol. 9, Thresholds of Nuclear Reactions Induced by Neutrons, Photons, Deuterons, Tritons, and Alpha Particles, September 1970.

- Vol. 10, Rev. 1, Tabulated Experimental Data for Neutron-Induced Interactions, July 1976.

- Vol. 11, Experimental Data, Indexes, and Techniques of Obtaining a Selected Set of Neutron Resonance Parameters, May 1972.

- Vol. 12, An Atlas of Resolved Neutron Resonance Parameters, July 1972.

- Vol. 13, An Atlas of Unresolved Neutron Resonance Parameters, September 1972.

- Vol. 14, TARTNP: A Coupled Neutron-Photon Monte Carlo Transport Code, February 1976.

- Vol. 15, Part A, The LLL Evaluated-Nuclear-Data Library (ENDL): Evaluation Techniques, Reaction Index, and Descriptions of Individual Evaluations, September 1975.

- Vol. 15, Part B, Rev. 1, The LLL Evaluated-Nuclear-Data Library (ENDL): Graphs of Cross Sections from the Library, October 1978.

- Vol. 15, Part C, The LLL Evaluated-Nuclear-Data Library (ENDL): Translation of ENDL NeutronInduced Interaction Data into the ENDF/B Format, April 1976.

- Vol. 15, Part D, Rev. 1, The LLL Evaluated-Nuclear-Data Library (ENDL): Descriptions of Individual Evaluations for $Z=0-98$, May 1978.

- Vol. 15, Part E, Data Testing Results for the LLL Nuclear Data Library (ENDL-78). August 1979.

- Vol. 15, Part F, Experimental and Evaluated Elastic Nuclear Plus Interference Cross Sections for Light Charged Particles, July 1980.

- Vol. 16, Rev. 2, Tabular and Graphical Presentation of 175 Neutron-Group Constants Derived from the LLL Evaluated-Nuclear-Data Library (ENDL), October 1978.

- Vol. 17. Part A, Rev. 2, Program LINEAR (Version 79-1): Linearize Data in the Evaluated-Nuclear-Data File/Version B (ENDF/B) Format, October 1979. 


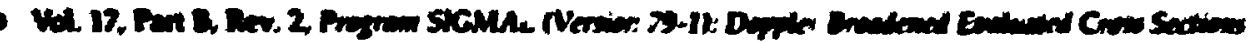

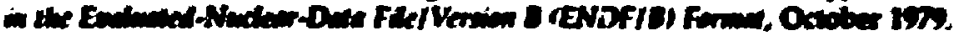

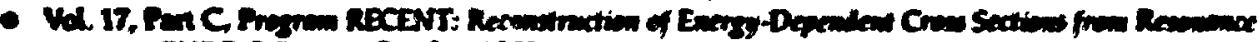
Punmeters in the ENDFIO Furmot, October 1979.

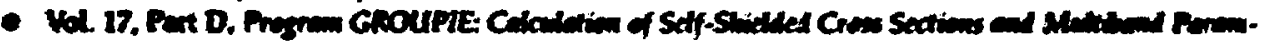

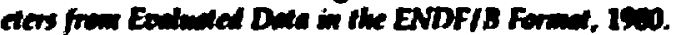

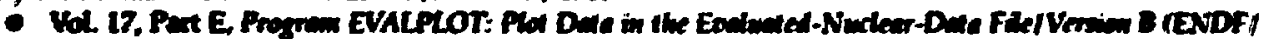
B) Finmat, Februmy 1979.

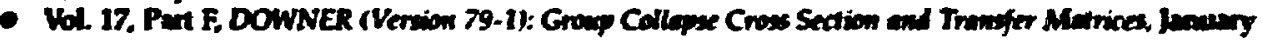
1979.

- Vat. 18, ACTR. Eanlueted Nextron Activation Cross-Section Livary, October 1978.

- Vol. 19. Neutron-Induced Angular end Energy Distributions: Graphical Experimental Dat, April 1977.

- Vov. 20, Bonderenko Self-Shielded Cross Sections and Multiburd Parametiors Derioed from the ULL Eoduated-Nuclear-Date Lbrary (ENDL), July 1978.

- Vol. 21, Part A, Marwell-Averaged Reactions Rates (Fo) for Selected Reactions between lons with Atomic Mass $\leq 11$, February 1979.

- Vol. 21, Part C. Program SIGMAL (Version 79-1): Doppler-Broadened Evaluated Cross Sections in the Livermore-Exaluated Nuclear Data Lbrary (ENDL) Format, March 1979.

- Vol. 22, Rev. 1, GAMIDEN: A Program to Aid in the ldentification of Unknown Materials by GammaRay Spectroscopy, June 1982.

- Vol. 23, ENSL and CDRL: Evaluated Nuclear Structure Libraries, February 1981.

- Vol. 23, Addendum, ENSL82 and CDRL82: The 1982 Version of Evaluated Nuclear Structure Libraries, lanuary 1983.

- Vol. 24, Thresholds and $Q$ Values of Nuclear Reactions Induced by Neutrons, Protons, Deuterons, Tntons, ${ }^{3}$ He lons, Alpha Particles, and Photons, March 1981.

- Vol. 25, Omega Documentation, August 1983. 


\section{Contientis}

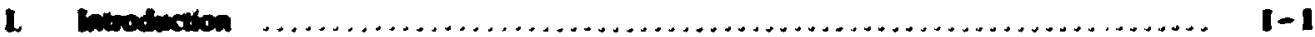

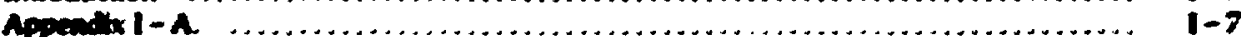

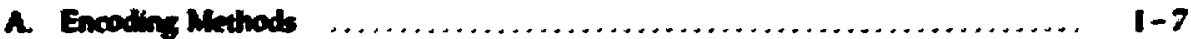

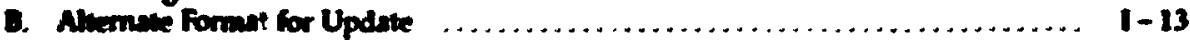

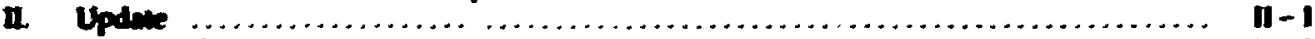

A Intoduction $\ldots \ldots \ldots \ldots \ldots \ldots \ldots \ldots \ldots \ldots \ldots \ldots \ldots \ldots \ldots \ldots \ldots \ldots \ldots \ldots \ldots \ldots$ It -3

B. Retuieval of Dat $\ldots \ldots \ldots \ldots \ldots \ldots \ldots \ldots \ldots \ldots \ldots \ldots \ldots \ldots \ldots \ldots \ldots \ldots \ldots \ldots \ldots \ldots \ldots$ II-4

C Dektion of Data $\ldots \ldots \ldots \ldots \ldots \ldots \ldots \ldots \ldots \ldots \ldots \ldots \ldots \ldots \ldots \ldots \ldots \ldots \ldots \ldots \ldots \ldots \ldots$ |l

D. Inserdion of Dra $\ldots \ldots \ldots \ldots \ldots \ldots \ldots \ldots \ldots \ldots \ldots \ldots \ldots \ldots \ldots \ldots \ldots, \quad$ II -8

E Making a New Lbrary a inilio $\ldots \ldots \ldots \ldots \ldots \ldots \ldots \ldots \ldots \ldots \ldots \ldots \ldots \ldots \ldots$ II-9

F. Output $\ldots \ldots \ldots \ldots \ldots \ldots \ldots \ldots \ldots \ldots \ldots \ldots \ldots \ldots \ldots \ldots \ldots \ldots \ldots \ldots \ldots \ldots \ldots \ldots \ldots \ldots$ II-9

II. ENDEP $\ldots \ldots \ldots \ldots \ldots \ldots \ldots \ldots \ldots \ldots \ldots \ldots \ldots \ldots \ldots \ldots \ldots \ldots \ldots \ldots \ldots \ldots \ldots \ldots \ldots \ldots$ II -1

A. Introduction $\ldots \ldots \ldots \ldots \ldots \ldots \ldots \ldots \ldots \ldots \ldots \ldots \ldots \ldots \ldots \ldots \ldots \ldots \ldots \ldots \ldots \ldots \ldots \ldots$ II -3

B. E and Residual Models $\ldots \ldots \ldots \ldots \ldots \ldots \ldots \ldots \ldots \ldots \ldots \ldots \ldots \ldots \ldots \ldots \ldots \ldots \ldots$ li -4

C. E Model Descriptions $\ldots \ldots \ldots \ldots \ldots \ldots \ldots \ldots \ldots \ldots \ldots \ldots \ldots \ldots \ldots \ldots \ldots \ldots$ III -7

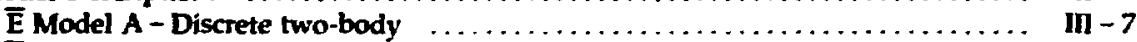

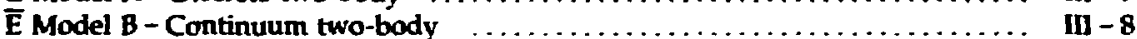

$\bar{E}$ Model C - Discrete two-body, continuum decay $\ldots \ldots \ldots \ldots \ldots \ldots \ldots \ldots \ldots \ldots$ II -9

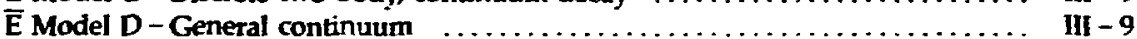

$\bar{E}$ Model E - Fission $\ldots \ldots \ldots \ldots \ldots \ldots \ldots \ldots \ldots \ldots \ldots \ldots \ldots \ldots \ldots \ldots$ III -9

$\bar{E}$ Model $\mathbf{F}-\mathrm{C}=\mathbf{1 2}, \mathbf{S}=\mathbf{8}$ or $\mathbf{1 0 . 1 1}$ for $y_{i} \ldots \ldots \ldots \ldots \ldots \ldots \ldots \ldots \ldots \ldots \ldots$

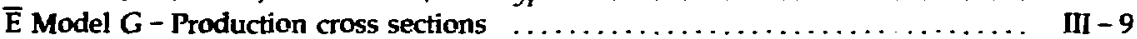

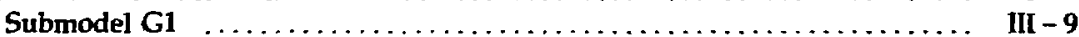

Submodel $\mathbf{G 2} \ldots \ldots \ldots \ldots \ldots \ldots \ldots \ldots \ldots \ldots \ldots \ldots \ldots \ldots \ldots \ldots \ldots$ III $-\mathrm{g}$

$\overline{\mathbf{E}}$ Type $\mathbf{H}-$ Gamma ray interaction cross sections $\ldots \ldots \ldots \ldots \ldots \ldots \ldots \ldots$ III $-\mathbf{I 0}$

Submodel $\mathrm{H} 1 \quad \ldots \ldots \ldots \ldots \ldots \ldots \ldots \ldots \ldots \ldots \ldots \ldots \ldots \ldots \ldots \ldots \ldots$ III -10

Submodel $\mathrm{H} 2 \quad \ldots \ldots \ldots \ldots \ldots \ldots \ldots \ldots \ldots \ldots \ldots \ldots \ldots \ldots \ldots \ldots \ldots$ III -10

Submodel $\mathrm{H} 3 \quad \ldots \ldots \ldots \ldots \ldots \ldots \ldots \ldots \ldots \ldots \ldots \ldots \ldots \ldots \ldots \ldots \ldots \ldots$ III -10

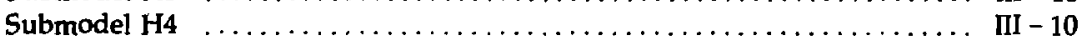

D. Residual Models $\ldots \ldots \ldots \ldots \ldots \ldots \ldots \ldots \ldots \ldots \ldots \ldots \ldots \ldots \ldots \ldots \ldots \ldots \ldots \ldots \ldots$ III -10

Residual Model 1 - Discrete two-body $\ldots \ldots \ldots \ldots \ldots \ldots \ldots \ldots \ldots \ldots \ldots$ III -10

Residual Model 2 - Continuum two-body $\ldots \ldots \ldots \ldots \ldots \ldots \ldots \ldots \ldots \ldots$ IIl -11

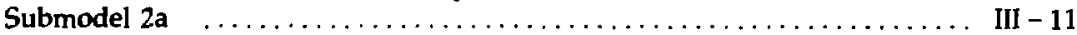

Residual Model 3 - Continuum two-body, continuum decay $\ldots \ldots \ldots \ldots \ldots \ldots$ III -12

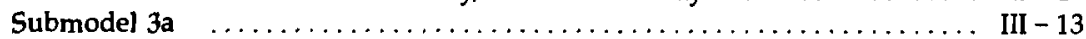

Residual Model 4 - Fission $\ldots \ldots \ldots \ldots \ldots \ldots \ldots \ldots \ldots \ldots \ldots \ldots \ldots$ III -13

Residual Model 5 - Discrete two-body, continuum decay $\ldots \ldots \ldots \ldots \ldots \ldots \ldots$ III -13

Residual Model 6 - Radiative capture $\ldots \ldots \ldots \ldots \ldots \ldots \ldots \ldots \ldots \ldots \ldots$ III -14

Residual Model 7 - Gamma ray induced reactions $\ldots \ldots \ldots \ldots \ldots \ldots \ldots$ III -15

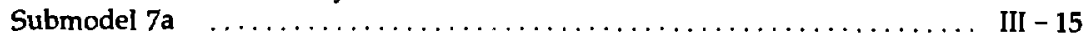

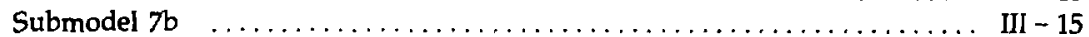

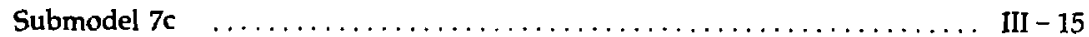

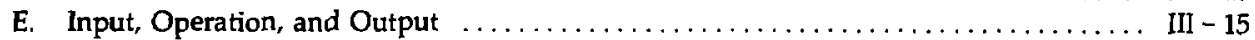

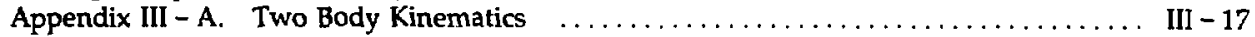

Appendix III - B. Decay Kinematics $\ldots \ldots \ldots \ldots \ldots \ldots \ldots \ldots \ldots \ldots \ldots \ldots \ldots \ldots \ldots \ldots$ III -21

Appendix III - C. Radiative Capture Kinematics $\ldots \ldots \ldots \ldots \ldots \ldots \ldots \ldots \ldots \ldots \ldots \ldots$ III -23

References $\quad \ldots \ldots \ldots \ldots \ldots \ldots \ldots \ldots \ldots \ldots \ldots \ldots \ldots \ldots \ldots \ldots \ldots \ldots \ldots \ldots \ldots \ldots \ldots$ IIJ -24

IV. GAMCHK $\ldots \ldots \ldots \ldots \ldots \ldots \ldots \ldots \ldots \ldots \ldots \ldots \ldots \ldots \ldots \ldots \ldots \ldots \ldots \ldots \ldots \ldots$ IV 1
A. Introduction
IV -3

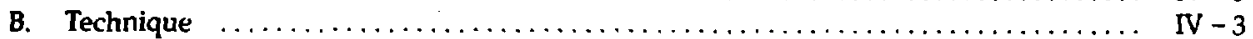
C. Input, Operation, and Output $\ldots \ldots \ldots \ldots \ldots \ldots \ldots \ldots \ldots \ldots \ldots \ldots \ldots \ldots \ldots$ IV -4 
$v \quad v$ y

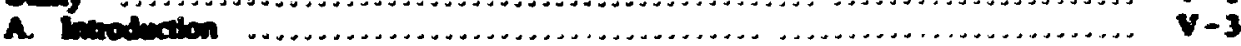

. Prepucetion of thput .............................................. v-3

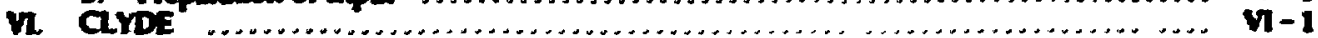

A. Introduction .................................................. $v$

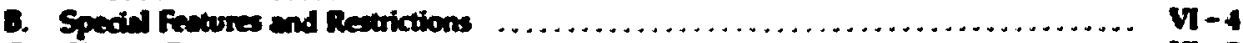

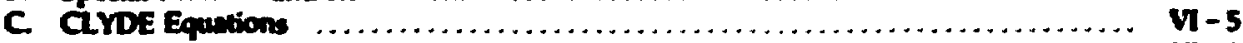

Tremder Matrix Definition ................................... $\mathbf{v}-\mathbf{5}$

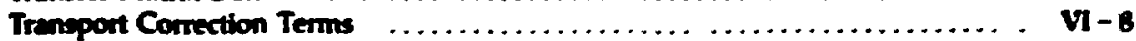

Average Group Flux and Inverse Speed ........................... VI-10

Reaction Group Average Cross Sections .......................... VI -11

Transfer Probability from Angular Distribution $(I-1)$ Data $\ldots \ldots \ldots \ldots \ldots . . \ldots$ V-12

General Relations $\ldots \ldots \ldots \ldots \ldots \ldots \ldots \ldots \ldots \ldots \ldots \ldots \ldots \ldots, \mathbf{V} \mathbf{V}-12$

The Inner Integral $\ldots \ldots \ldots \ldots \ldots \ldots \ldots \ldots \ldots \ldots \ldots \ldots \ldots \ldots, \mathbf{v}-15$

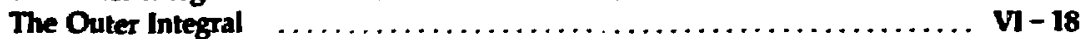

Transfer Probability from Tabular Energy Distribution $(I=4)$ Data $\ldots \ldots \ldots \ldots$ VI-19

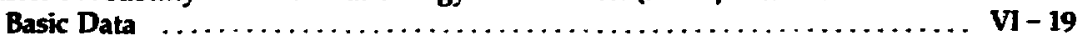

Unit Base Transformation $\ldots \ldots \ldots \ldots \ldots \ldots \ldots \ldots \ldots \ldots \ldots \ldots \ldots \ldots \ldots$, VI -19

The Inner Integral $\ldots \ldots \ldots \ldots \ldots \ldots \ldots \ldots \ldots \ldots \ldots \ldots \ldots \ldots \ldots \ldots \ldots \ldots, \mathbf{v}-\mathbf{2 1}$

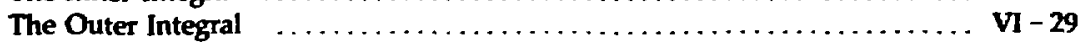

Energy Production and Deposition $\ldots \ldots \ldots \ldots \ldots \ldots \ldots \ldots \ldots \ldots, \mathbf{v}-\mathbf{3 0}$

Isotope Production $\ldots \ldots \ldots \ldots \ldots \ldots \ldots \ldots \ldots \ldots \ldots \ldots \ldots \ldots \ldots \ldots \ldots, \mathbf{v}-31$

D. CLYDE Input Format and Instructions $\ldots \ldots \ldots \ldots \ldots \ldots \ldots \ldots \ldots \ldots \ldots \ldots \ldots \ldots \ldots \ldots \ldots \ldots$, VI -31

E. CLYDE Output Format and Instructions $\ldots \ldots \ldots \ldots \ldots \ldots \ldots \ldots \ldots \ldots \ldots \ldots \ldots \ldots \ldots \ldots$, VI -37

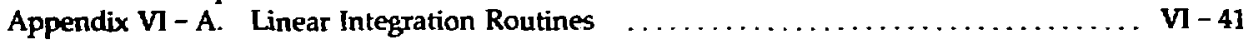

Appendix VI - B. Numerical Integration $\ldots \ldots \ldots \ldots \ldots \ldots \ldots \ldots \ldots \ldots \ldots \ldots \ldots \ldots \ldots \ldots \ldots$, VI -43

Appendix VI - C. Constants Used in Integration $\ldots \ldots \ldots \ldots \ldots \ldots \ldots \ldots \ldots \ldots \ldots \ldots \ldots \ldots \ldots \ldots$, VI -45

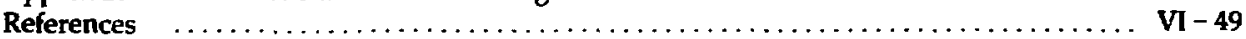

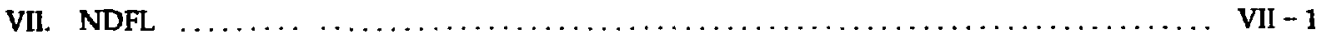

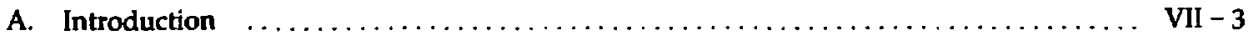

B. File Description and Usage $\ldots \ldots, \ldots \ldots \ldots \ldots \ldots \ldots \ldots \ldots \ldots \ldots \ldots \ldots$, VII -3

C. Input, Operation, and Output $\ldots \ldots \ldots \ldots \ldots \ldots \ldots \ldots \ldots \ldots \ldots \ldots \ldots \ldots \ldots \ldots \ldots \ldots \ldots$, VII -4

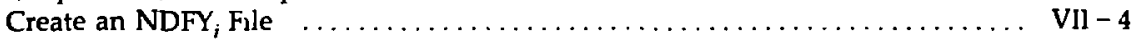

Update an NDFY, File $\ldots \ldots \ldots \ldots \ldots \ldots \ldots \ldots \ldots \ldots \ldots \ldots \ldots \ldots \ldots \ldots$, VII -5

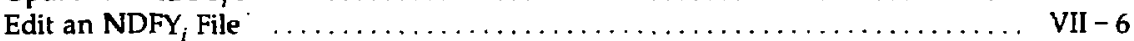

Appendix VII - A. Physical Layout of a Binary NDFY ${ }_{i}$ File $\ldots \ldots \ldots \ldots \ldots \ldots \ldots$ VII -11

A. Global Section $\ldots \ldots \ldots \ldots \ldots \ldots \ldots \ldots \ldots \ldots \ldots \ldots \ldots \ldots \ldots \ldots \ldots \ldots \ldots \ldots, 11$

B. Individual Materials - Directory and Descriptor Blocks (ISODEF) $\ldots \ldots \ldots \ldots$ VII -12

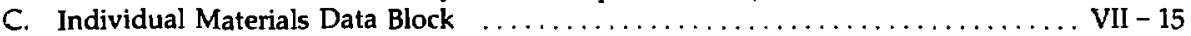

Appendix VII - B. Physical Layout of the BDFLS ASCII File $\ldots \ldots \ldots \ldots \ldots \ldots \ldots$ VII -17

Appendix VII - C. Group Collapse of the NDFY Files $\ldots \ldots \ldots \ldots \ldots \ldots \ldots \ldots \ldots$ VII -19

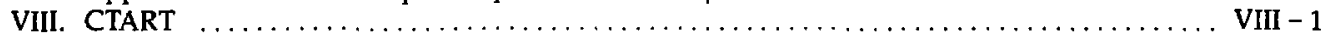

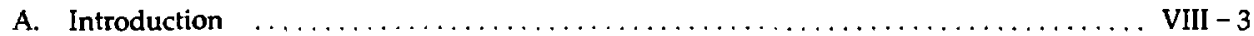

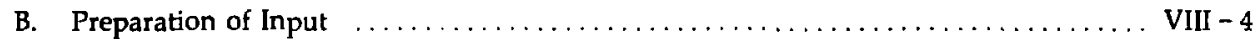

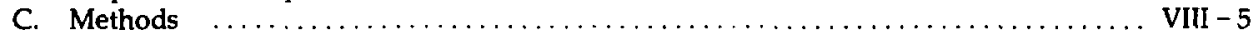

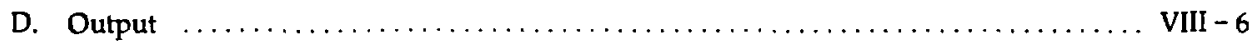

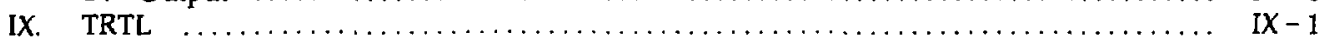

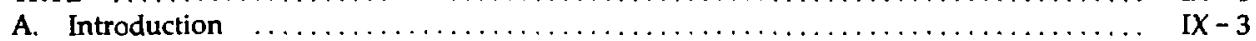

Appendix IX - A. $\quad \ldots \ldots \ldots \ldots \ldots \ldots \ldots \ldots \ldots \ldots \ldots \ldots \ldots \ldots \ldots \ldots \ldots \ldots \ldots \ldots \ldots \ldots \ldots$, IX -5

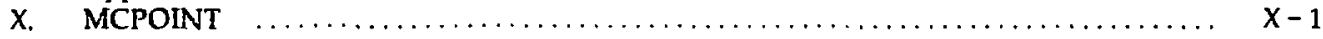
A. Introduction $\ldots \ldots \ldots \ldots \ldots \ldots \ldots \ldots \ldots \ldots \ldots \ldots \ldots \ldots \ldots \ldots \ldots \ldots \ldots, \quad x-3$

B. Conversion of Data of MCNP Format $\ldots \ldots \ldots \ldots \ldots \ldots \ldots \ldots \ldots \ldots \ldots \ldots \ldots \ldots \ldots$ 
CHa PTER L:

INTRODUCTION

$$
1-1 / 1-2
$$




\section{INTRODUCTON}

\section{UAlEGA is a CRAY I computer program that controls nine codes ued by the LLNL Plysicel vate Groep for:}

1. Updating the libraries of evaluated data maintained by the group (UPUATE).

2 Calculating average values of encrgy deposited in secondary particles and residual nuclei (ENDEP).

3. Checking the libraries for internal consistency, especially for energy conservatior (GAMCHK).

4. Producing listings, indexes and plots of the library data (UTILITY).

5. Producing calculational constants such as group averaged cross sections and transfer matrices for diffusion and $\mathbf{S}$ transport codes (CLYDE).

6. Producing and updating standard files of the ealculational constants used by LLNL Sn and diffusion transport codes (NDFL).

7. Producing calculational constants for Monte Carlo transport codes that use group-averaged cross sections and continuous energy for particles (CTART).

8. Produeing and updating standard files used by the LLNL Monte Carlo transport codes (TR TL).

9. Producing standard files used by the LANL pointwise Monte Carlo transport code MCNP (MCPOINT).

The first four of these functions and codes deal with the libraries of evaluated data and the last five with various aspects of producing calculational constants for use by transport codes. In 1970 a series, called PD memos, of internal and informal memoranda was begun. These were intended to be circulated among the group for comment and then to provide documentation for iater reference whenever questions arose about the subject matter of the memos. They have served this purpose and now will be drawn upon as source material for this more comprehensive report that deals with most of the matters covered in those memos. In addition to the PD memos, several other of the volumes of this series (UCRL 50400) deal with many of the subjects dealt with in this volume. For the convenience of the reader some developments will be repeated in part or whole. The criterion of how much technical discussion will be included is: whatever is needed to understand what the various codes do and why they do it. Since the nine codes have quite different functions, this results in significantly varied quantities of discussion for each code. The basic rule is that when in doubt, as to whether discussion of some aspect of what a code does is needed, include it. 
Throughont this volmene reference b asde to certain quantities uning their short or juczon nanes. This eliminates repetition of relutively lons deacriptive names and thus wackes the discuscions flow more snoothly. Eech of these quantities is described in the following list:

\begin{tabular}{|c|c|}
\hline short Name & Definition \\
\hline $\mathbf{Z A}$ & $\begin{array}{l}\text { Designation of a nucleus by charge and mass } \\
\left(1000^{*} Z+A\right) \text {. For elements having inixtures of isotopes } \\
A \text { is taken to be } 0 \text {. Thus, natural chlorine has } Z A= \\
17000 \text {. }\end{array}$ \\
\hline $\mathbf{Y i}$ & $\begin{array}{l}\text { Incident particle in a reaction. For definition of values } \\
\text { see Appendix A. }\end{array}$ \\
\hline Yo & $\begin{array}{l}\text { Secondary particle from a reaction. For definition of } \\
\text { values see Appendix A. }\end{array}$ \\
\hline $\mathrm{C}$ & $\begin{array}{l}\text { Reaction descriptor numerical equivalent. e.g., } C=12 \\
\text { signifies a reaction resulting in two secondary } \\
\text { neutrons. For definition of values see Appendix } A \text {. }\end{array}$ \\
\hline I & $\begin{array}{l}\text { Reaction property numerical equivalent. e.g., I=0 } \\
\text { signifies the cross section. For definition of values see } \\
\text { Appendix A. }\end{array}$ \\
\hline $\mathbf{S}$ & $\begin{array}{l}\text { Reaction Modifier numerical equivalent. e.g., } S=1 \\
\text { signifies that the reaction proceeds through a level in } \\
\text { an intermediate nucleus. For definition of values see } \\
\text { Appendix A. }\end{array}$ \\
\hline$X \mathbf{i}$ & $\begin{array}{l}\text { Numerical values }(i=1,4) \text { required to complete } \\
\text { definition of non-zero } S \text {-values. e.g., for } S=1 \text { (see } \\
\text { above) the energy of the level is entered in the } X 1 \\
\text { field. For definition of the values to be entered in the } \\
X i \text { fields for each } S \text {-value see Appendix } A \text {. }\end{array}$ \\
\hline
\end{tabular}

The calling sequence for any of the codes controlled by OMEGA is:

OMEGA (code) (input file) (box no.) (hsp option) (library name) 0 (output file name) (output file size) / T V

All of the quantities enclosed in parentheses are defined below. Note that the parentheses only indicate a problem dependent value. The parentheses do not appear in the calling sequence. An example of using OMEGA to run UPDATE using file INUP with a library called ND821201 is:

$$
1-4
$$




\section{The various quntities in parentheses have the following significances \\ code \\ This is the name of the code (one of the nine listed above) that is being run under OMEGA's control.}

input file

box no.

hsp option

library name

0

output file name

output file size the file required by "code" for input

user's box number

Each "code" produces output that is descriptive and/or diagnostic for the problem. This output may be directed to the FR 80 (use "fr80" for option), the high speed printer (use "hsp" for option), or kept for inspection (use "debug" for opticil) and/or output to RJET or other medium using ALLOU'T.

the library file that is being used for updating or other purposes. If a new library is being made ab initio, the word "none" is entered here (see chapter II). For the TRTL code a standard file, DT041773, is used if a totally new file is being made (see chapter IX).

The zero in this position indicates that the library exists as a file. The zero must be included in the calling sequence.

the name of the file to be created as output

the size of the output file to be created in units of 100000 words. In the example above, the output file will be created as 2000000 words. When the code is finished it will shrink the file to the size needed.

In that which follows, the calling sequence for each code will be described in more detail, including specifications for the form and significance of the input file that is specific to the code being run under OMEGA's control. 
Thure ere four generie libraries of evaluated data on which the codes controlied by OMEGA qperate. They are

ENDL

Evaluated Neutron Data Library that contains the data essociated with neutron induced reactions, including cross sections, angular distributions, energy distributions, particle multiplicities, and average energy deposits. All evaluations contained in ENDL are coinplete in the sense of containing data for all energetically possible reactions for incident neutron energies from $10^{* *}-10$ to 20 . $\mathrm{MeV}$, if the reaction cross section is judged to rise to, at least, 10 millibams somewhere in that neutron en:ergy range.

ACTL

Neutron Activation Cross Section Library that contains eross section data for activation of specifie nuclides by neutron interaction. The cross section data are for those reactions for which interest has been identified by a user of the data. Thus, the ACTL file is, by design, incomplete but infinitely hospitable to addition of new reactions as interest is identified.

ECPL

Evaluated Charged Particle Library that contains the data associated with charged particle induced nuclear reactions, including cross sections, angular and energy distributions of secondary particles, particle multiplicities, average energy deposits, in-flight cross sections for hot targets, and maxwell-averaged data for hot media.

EGDL Evaluated Gainma-Ray Interaction Data Library that contains data for coherent scattering, incoherent scattering, photoelectric and pair-production processes for gamma-ray energies from .0001 to 100 . MeV. This library contains no photo-nuclear reaction data. Thus, it is appropriate to the interaction of photons with the elements as cold targets and as atoms. Targets are included from $\mathrm{Z}=1$ to $\mathrm{Z}=100$. 


\section{APPENULX1-A}

\section{A. ENCONING METHODS}

A common practice throughout this volume is to refer to the characteristies of muclear reactions using a shorthand or symbolic representation. This eliminates repetition of relatively long descriptive names that can becoine tedious for the reader. The quantities for which such representation is made are:

Short Name Definition

ZA

$Y \mathbf{i}$

Yo

C

I

$\mathbf{S}$

$\mathrm{Xi}$
Designation of a nucleus by charge and mass $(1000 * Z+A)$. For elements having mixtures of isotopes, $A$ is taken to be 0 . Thus natural chlorine has a $Z A$ of 17000 .

Incident particle in a reaction. For definition of values see Table 1 .

Secondary particle from a reaction. For definition of values see Table 1 .

Reaction descriptor numerical equivalent. e.g., $\mathrm{C}=12$ signifies a reaction resulting in two secondary neutrons. For definition of values see Table 2 .

Keaction property numerical equivalent. e.g., l=0 signifies the cross section. For definition of values see Table 3 .

Reaction Modifier numerical equivalent. e.g., $S=1$ signifies that the reaction proceeds through a level in an intermediate nucleus. For definition of values see Table 4.

Numerical values $(i=1,4)$ required to complete definition of non-zero S-values. e.g., for $S=1$ (see above) the energy of the level is entered in the $X 1$ field. For definition of the values to be entered in the Xi fields for each $S$-value see Table 4. 
TABLE 1-A.l. Particle Designator, y.

\begin{tabular}{ll}
\hline $\mathbf{y}$ & \multicolumn{1}{c}{ Particle } \\
\hline $\mathbf{1}$ & $\mathbf{n}$ \\
$\mathbf{2}$ & $\mathbf{p}$ \\
3 & $\mathbf{d}$ \\
4 & $\mathrm{t}$ \\
$\mathbf{5}$ & ${ }^{3} \mathrm{He}$ \\
$\mathbf{6}$ & $\boldsymbol{\alpha}$ \\
7 & $\gamma$ \\
8 & $\beta^{+}$ \\
9 & $\beta^{-}$ \\
10 & EC \\
11 & Neutron as residual nucleus \\
12 & Proton as residual nucleus \\
13 & Deuteron as residual nucleus \\
14 & Triton as residual nucleus \\
15 & ${ }^{3}$ He as residual nucleus \\
16 & $\alpha$ as residual nucleus \\
\hline
\end{tabular}

a Used to identify both the incident particle, $y_{i}$, and the outgoing particle, $y_{0}$, to which any distribution data (e.g., energy, angle) pertain: $y_{i}=0-7$ only; $y_{0}=0-16$.

$b$ If no property (e.g., energy distribution) is given the $y_{0}$ is zero or left blank in input. If $y_{i}$ is zero or blank, the data which follow are nuclear structure data. 


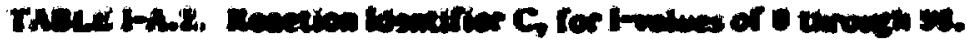

\begin{tabular}{|c|c|c|}
\hline Wind of vate & $\mathbf{c}$ & menetion type \\
\hline 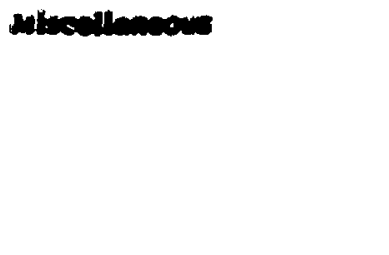 & $\begin{array}{l}1 \\
2-7 \\
3 \\
10\end{array}$ & 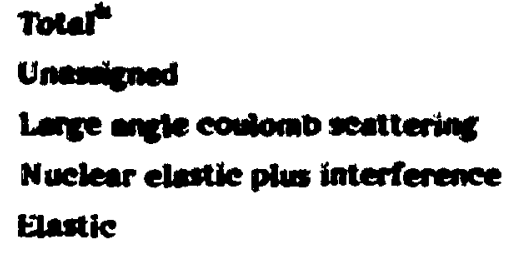 \\
\hline Neutrose + cammas only & $\begin{array}{l}11 \\
12 \\
13 \\
14 \\
15 \\
16-19\end{array}$ & $\begin{array}{l}\left(y_{i}, n^{2} \gamma\right) \\
\left(y_{p}, 2 n \gamma\right) \\
\left(y_{i}, 3 n \gamma\right) \\
\left(y_{i}, 4 n \gamma\right) \\
\left(y_{i}, x f\right) \text { total fission } \\
\text { Unassigned }\end{array}$ \\
\hline $\begin{array}{l}\text { Neutrons + charged } \\
\text { particles + gammas }\end{array}$ & $\begin{array}{l}20 \\
21 \\
22 \\
23 \\
24 \\
25 \\
26 \\
27 \\
28 \\
29 \\
30 \\
31 \\
32 \\
33-36\end{array}$ & 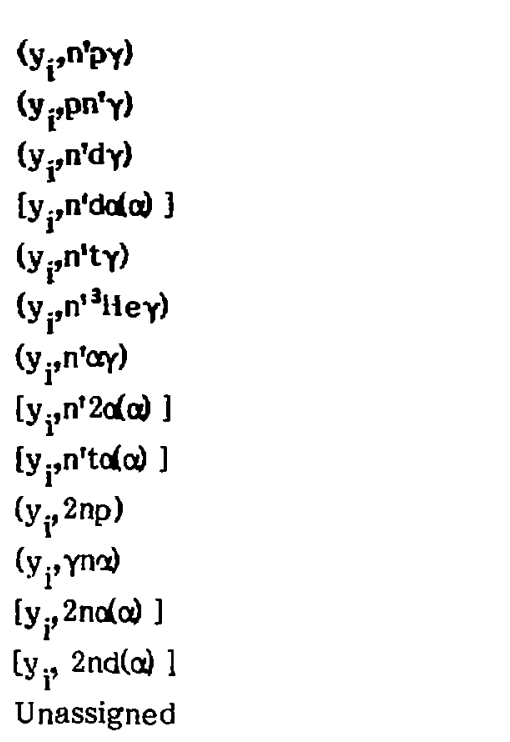 \\
\hline $\begin{array}{l}\text { Charged particle and/ } \\
\text { or gamma formation }\end{array}$ & $\begin{array}{l}37 \\
38 \\
39 \\
40 \\
41 \\
42\end{array}$ & $\begin{array}{l}{\left[y_{i}, 2 \alpha(\alpha)\right]} \\
{\left[y_{i},{ }^{3} \operatorname{He} \alpha(\alpha)\right]} \\
{\left[y_{i}, p t(\alpha)\right]} \\
\left(y_{i}, p \gamma\right) \\
\left(y_{i}, d \gamma\right) \\
\left(y_{i}, t \gamma\right)\end{array}$ \\
\hline
\end{tabular}




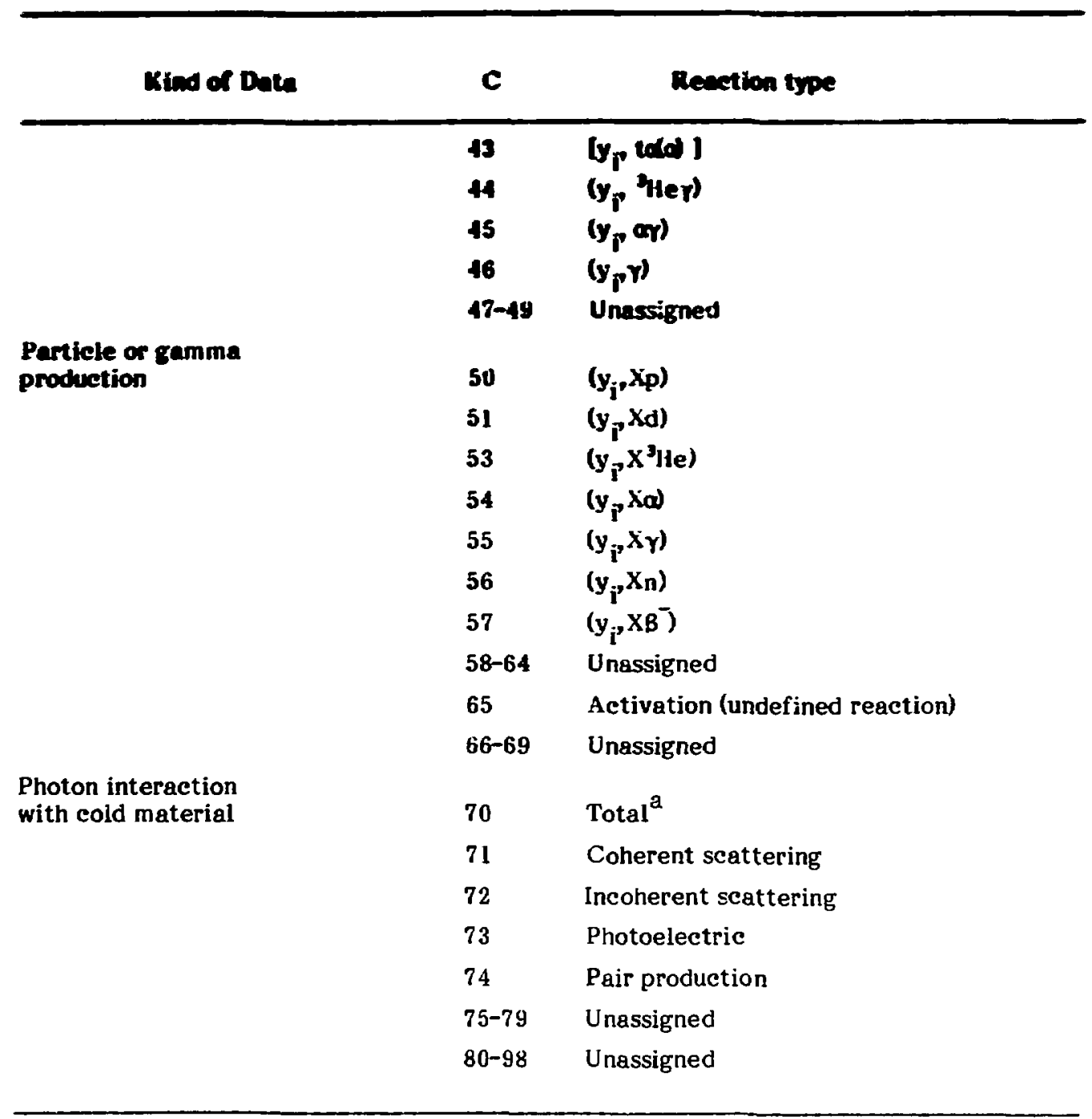

a Not stored in the system, but obtained for output display and transmission format by combining other data. 


\begin{tabular}{|c|c|c|}
\hline 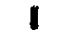 & Mowetion Property & Defiecition \\
\hline - & Inctrated erows sections (b) & a(c) \\
\hline 1 & Angular distributions, normalized probabilities (per unit coine) & $P(t-1)$ \\
\hline 3 & Ënersy-angle distributions, norinalized probstilities" & $\mathbf{F}(\mathbf{t}-\mathbf{1 1}, \mathbf{t})$ \\
\hline 4 & $\begin{array}{l}\text { tinergy-angle distriburions, normalized legendre coefficients. } \\
I^{4}(t E+E)=p(E+E) \text {, the usual normalized energy }\end{array}$ & \\
\hline & probability (per MeV) & 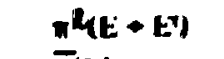 \\
\hline 7 & A verege number of neutrons per fission (prompt or delayed) & $\overline{\mathbf{V} E)}$ \\
\hline 8 & Histogram form of energy distribution & S PE, ETdE \\
\hline 9 & Photon or particle multiplicity & $\mathbf{M}(E)$ \\
\hline 10 & A verage energy of a secondary particle (MeV) & $E^{\prime}\left(y_{0}, E\right)$ \\
\hline 11 & A verage energy of a residual nucleus (MeV) & $E(R, E)$ \\
\hline 80 & Maxwell average reaction rates $(\mathrm{b}-\mathrm{cm} / \mathrm{sh})$ & ov $(k T)$ \\
\hline 81 & In-flight (Doppler-broadened) cross sections (b) & $\alpha k T, E)$ \\
\hline 84 & Maxwell-averaged energy distributions (per MeV) & $\mathbf{P}\left(\mathbf{k T}, E^{\prime}\right)$ \\
\hline 89 & Maxwell-averaged photon or particle multiplicity & $M(k T)$ \\
\hline 90 & $\begin{array}{l}\text { Maxwell-averaged total average energy of secondary } \\
\text { particle (MeV) }\end{array}$ & $E^{\prime}\left(y_{0}, k T\right)$ \\
\hline 91 & Muxwell-uveraged average energy of residual nucleus (MeV) & $E^{\prime}(R, k T)$ \\
\hline 92 & Waxwell-averaged total average energy of reacting particles (MeV) & $E\left(y_{j}, k^{\prime} T\right)$ \\
\hline
\end{tabular}

a lie illustrate the use of these designations by constructing a reaction code for the energy distribution of protons from the ${ }^{59} \mathrm{Ni}\left(n, n^{\prime} p\right)^{57}$ Co reaction:

Incident particle:

Reaction property:

Keaction type:

Outgoing particle whose property is recorded:

Reaction modifier:

$Q_{0}$ of reaction:

'Ihis gives for the reaction code

$\begin{array}{lll}\mathrm{Yi} & \mathrm{I} & \mathrm{C} \\ 0 \mathrm{l} & 04 & 20\end{array}$

$\begin{array}{ll}\mathrm{C} & \text { Yo }_{\mathrm{O}} \\ 20 & 02\end{array}$

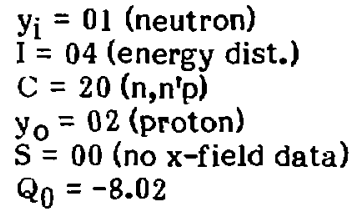

b Angular data are expressed in the center-of-mass $(\mathrm{cm})$ system for all two-body breakups. Wultibody breakups are, of course, in the Laboratory system.

* $1=1$ data must be present when $1=3$ data are given for a reaction with identical incident energy and cosine meshes. While the inclusion of $I=1$ data with $1=3$ data for a reaction is not a necessary condition for representing energy-angle correlated data, we have chosen this mode of representation for convenience of changing the data and for eonsistency. 


\begin{tabular}{|c|c|c|}
\hline 3 & Reaction Parencter & $x$ freld deforition \\
\hline intix & No $x$-tived date & -- \\
\hline ora & Level exeltation & $w_{i}($ WeV $)$ \\
\hline $\mathbf{0 2}$ & $\begin{array}{l}\text { Pre-equilibrium and unresolved direct internetion } \\
\text { procenes }\end{array}$ & None \\
\hline 03 & Gamma ray production & E $(M e V)$ \\
\hline 05b & Aetivation & $\begin{array}{l}(\mathrm{ZA})_{2}, \mathrm{w}_{2}(\mathrm{MeV}) \\
\text { half-life }(s)\end{array}$ \\
\hline u7 & Delayed group half-lives & $\tau_{1 / 2}(s)$ \\
\hline 08 & Time sequential cluster model & $w_{1}(\mathrm{MeV})$ \\
\hline 10 & Wide level excitation & $W_{1}(\mathrm{MeV}), \Gamma_{1}(\mathrm{Mev})$ \\
\hline 11 & $\begin{array}{l}\text { Second particle from wide level time-sequential } \\
\text { reaction }\end{array}$ & $w_{2}(\mathrm{MeV}), \Gamma_{2}(\mathrm{MeV})$ \\
\hline 15 & Density and Temperature Dependence & a $T(k e V)$ \\
\hline
\end{tabular}

a $\mathrm{X}_{1}$ corresponds to the initial nucleus, $\mathrm{X}_{2}$ to the second nucieus in a sequence of deexcitations, etc.

b $(\mathrm{ZA})_{2}=100 \mathrm{Z}_{2}+\mathrm{A}_{2}$ of product nucleus. 


\section{K ALTinNATE POLWAT PON UPOATE}

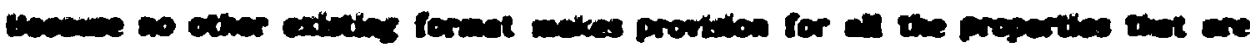

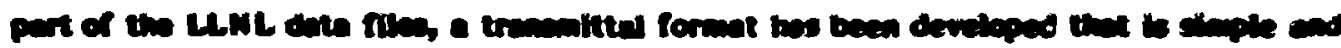

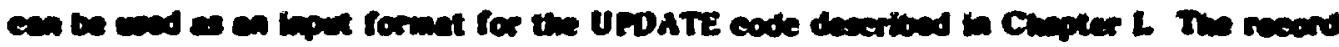

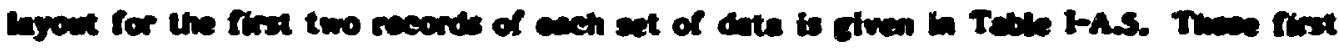

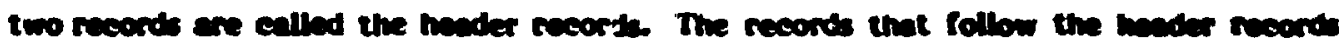

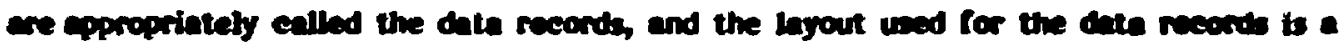
function of the reaction property ("I-vilue") described in the second of the two bader recond. Only four types of layouth, or organizations, are required to mancle the data for the 16 t-values defined in Table H-A.3.

TABLE HA.5. Field definitions for header reconds.

\begin{tabular}{|c|c|c|c|c|}
\hline & Columns & Format & Name & Description \\
\hline \multirow[t]{8}{*}{ Record 1} & $1-6$ & I6 & $\mathbf{Z A}$ & $1000 Z+A$ \\
\hline & 8-9 & I2 & $\mathbf{y}_{\mathbf{i}}^{\mathbf{a}}$ & Incident particle descriptor. \\
\hline & $11-12$ & $\mathbf{I 2}$ & $\mathbf{Y}_{\mathbf{0}}^{\mathbf{a}}$ & Outgoing particle deseriptor. \\
\hline & $14-24$ & E11.4 & $\mathbf{A}$ & Atomic mass for this ZA (amu). \\
\hline & 26-31 & 16 & DATE & Date last ehanged. \\
\hline & 34 & 11 & Flag & Enter a " 4 " to indicate type of format to follow. \\
\hline & $36-46$ & E11.4 & & Level energy of the target (MeV). \\
\hline & 48-58 & Ell 1.4 & & Half-life of the target(s). \\
\hline \multirow[t]{7}{*}{ Kecord 2} & $1-2$ & 12 & $\mathrm{c}^{\mathrm{a}}$ & Reaction number. \\
\hline & $3-5$ & I3 & $1^{\mathrm{a}}$ & Reaction property designator. \\
\hline & $6-8$ & 13 & $\mathbf{s}^{\mathbf{a}}$ & Reaction modifier flag. \\
\hline & $10-20$ & E11.4 & $\mathrm{Q} 0^{\mathrm{Q}}$ & Mass difference $Q$ for the reaction. \\
\hline & $22-32$ & Ell.4 & $\mathrm{x}_{1}^{\mathrm{a}}$ & Value depends on the value of $S$. \\
\hline & $34-44$ & Ell.4 & $x_{2}^{a}$ & Value depends on the value of S. \\
\hline & $46-56$ & E11.4 & $\mathrm{x}_{3}^{\mathrm{a}}$ & Value depends on the value of $\mathrm{S}$. \\
\hline
\end{tabular}

A more complete definition of these fields can be found in Tables l-A.l to 1-A.4. 


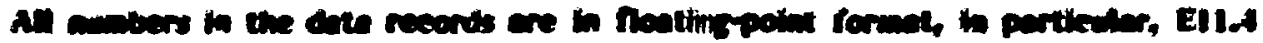

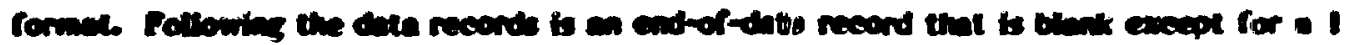

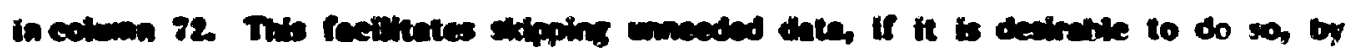

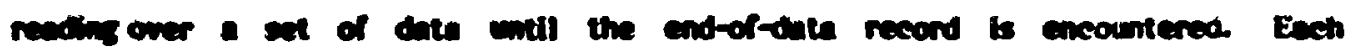

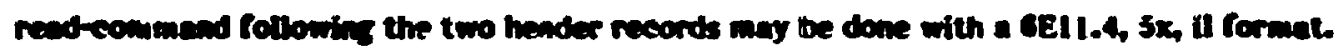

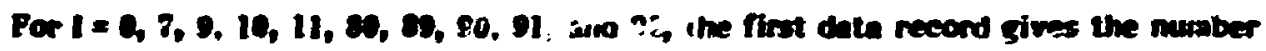
of pats of independent/dependert variables to follow. The signiffeance of the independent and dependent varisbles is given in Table II-: for each I-value.

For 1 = 1, 8, 81, and 84, the first recond gives the musber of values of the factorable parameter (incident particle enezy for $1=1,8 ; \mathrm{kT}$ for $1=81,84$ ). The following data records are given in sets, with the number of sets equel to the value given in the first-record described above. Each set has a first record that gives the value of the parameter ( $E$ or $K T$, followed by the number of pairs of independent/dependent variables, which are defined in Table II-2 for each 1-value.

For I = 3 there are two factorable parameters fincioient particle energy and cosine of the angle of emission in the laboratory system). The first data record gives the number of incident particle energies for which $1=3$ data are given. The second data record gives the value of the first energy and the number of cosines for the first energy. The third data record has the first cosine for the first energy and the number of cosine-secondary energy pairs to follow for the incident particle energy given in data record 2 and this cosine. Data records 4 et seg. contain the cosine-secondary energy data for the first energy-first cosine. Data records 3 through 4 (et seq.) are repeated for the number of cosines given in data record 2. This procedure is repeated for the number of incident particle energies given in the first data record.

For $I=4$ there are two parameters ( $\ell$ and $E)$. The first data record gives the number of $\ell$-values (sets of Legendre polynominal coefficients) that follow. For each set of coefficients, the first record gives the order of $\ell$, followed by the number of incident-particle energies for which secondary-energy/coefficient pairs are given. For each incident-particle energy there is a record that gives the incident-particle energy and the number of pairs of secondary-energy/coefficient pairs that follow. The remaining data for that subset are the secondary-energy/coefficient values for that order of Legendre polynominals and that incident-particle energy. 


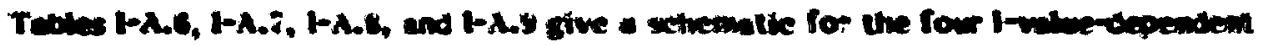
byous.

TAuLE I-A.6. Fiedd defindions for I = 0,7, y, $37,11,00,09,90,91$ and 92

\section{Hecond}

1

First header record, see Table 1-A.5.

2

Second header record, see Table 1-A.5.

3 Number of pairs of independent/dependent variables, e.g., energyfeross section (El 1.4).

4 et seq. Three pairs per record (6E 11.4$)$ of independent/dependent variables.

Last End-of-data record (1 in column 72).

TABLE I-A.7. Fieid definitions for $1=1,8,81,84$.

Record

$1 \quad$ First header record, see Table I-A.5.

$2 \quad$ Second header record, see Table I-A.5.

3 Number of values of the factorable parameter (NF) (El 1.4).

( $E$ for $I=1,8, k T=$ for $I=81,84$ ).

$4^{\mathrm{a}} \quad$ Value of the factorable parameter, number of pairs of independent/ dependent variables that follows (2Ell.4).

5 et seq. ${ }^{a}$ Three pairs per record $(6 \mathrm{E} 11.4)$ of independent/dependent variables [e.g., for $I=1, \mu, P(E, \mu)$ ].

Last End-of-data record ( 1 in column 72).

a Repeated NF times. 
Meeond

$\mathbf{1}$

First header recond, see Table I-A.5.

2

Second header record, see Table I-A.5.

3

Number of incident particle energies NE, (EII.4).

4 a

First incident particle energy, number of cosines for this energy (2E1 1.4), NC.

5b Value of the first cosine, number of cosine-secondary energy pairs to follow (2E1 1.4).

6 et seg-a Three pairs per record of secondary energies and probabilities.

Last End-of-data reeord (1 in column 72).

\begin{abstract}
a Repeated NE times.
b Kepeated NC times.
\end{abstract}

TABLE 1-A.9. Field definitions for $I=4$.

\title{
Record
}

1

First header record, see Table 1-A.5.

2 Second header record, see Table I-A.5.

3 Number of values of $\ell$ (E1 I.4), NI.

4a Value of $\ell$, number of incident energies for this $\ell, N E,(2 E 11.4$ ).

$5^{b} \quad$ Incident energy; number of secondary energy coefficients to follow (2E11.4).

6 et seg. ${ }^{a} \quad$ Three pairs per record of secondary energies and coefficients (6E1 1.4).

Last End-of-data record ( 1 in column 72).

\footnotetext{
a Repeated NL times.

b Repeated NE times.
} 
CHA PTEK II:

UPUATE

$\mathrm{II}-1 / i \dot{i}-2$ 


\title{
D. UPDATE:
}

\section{A. INTHODUCTon}

UPUATE bs a code that retrteves date, deletes datu or thiserts data into any one of the eviluated bbreries animinined by the Pinsical Data Group. The ealling sequence for ruming UPUATE ix

\author{
UMEGA UPUATE (input file) (box no.) (hsp option) (library name) \\ o (output file name) (cutput file size) / TV
}

The parentheses denote problem dependent quantities and do not appear in the calling sequence. An example of running UPDATE using an input file INUP with a library called NU821201 to make a library ND821202 is: omega update inup t64 fr80 nd821201 0 nd821202 $20 / .9 .999$

The various quantities in parentheses have the following significance:

input file

box no.

hsp option

library name

0

output file narne

output file size the file required by U PDATE for input

user's box number

UPDATE produces output that is descriptive and/or diagnostic for the problem. This output may be directed to the FR80 (use "fr80" for option), the high speed printer (use "hsp" for option), or kept for inspection (use "debug" for option) and/or output to RJET or other medium using ALLOUT.

the library file that is being used for updating or other purposes. If a new library is being made ab initio, the word "none" is entered here. See below.

The zero in this position indicates that the library exists as a file. The zero must be included in the calling sequence.

the name of the file to be created as output. This may be a file of retrieved data or a new library.

the size of the output file to be created in units of 100000 words. In the example above, the output file will be created as 2000000 words. When the code is finished it will shrink the file to the size needed. 


\section{B. RETIIEVAL UP DATA}

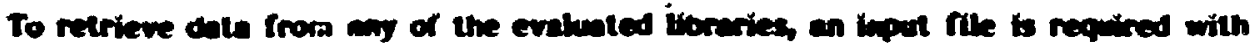
two sets of three reconds for ench retrleme. Any nuwoer of retirievals may be dowe but for each retrieval tivere must be retrieval miteria speetfies. The first record of each set (turcet recond) specifies the limits of the range of isotopes or elerpents, the incident and secondary particles of interest, and a value of 3 or 5 in column 34 that specifies the output mode of the retrieval. A value of 3 indicates that the cutput of the retrieval is to be in a format having one entry per record for the retrieved data. A value of 5 indicates that the output is to be in a format (transmission format) with multiple entries per record and common values factored out of the data. The second recond of each set (reaction record) specifies the limits of the range of reaction descriptors and the third record of each set (data record) gives the limits of the energy range of the independent variables for which the retrieval is to be made. The specifications of the fields of the first two records can be found in Trble Il.1. For the third record the formats and specifications of the retrieved data are found in Table 11.2 , if the value of the flag in column 34 of the first record is 3. For a value of 5 in column 34 of the first record, the formats and specifications of the retrieved data are given in Tables 1-A.6, I-A.7, 1-A.8, and 1-A.9 of Appendix 1-A.

\section{DELETIUN OF DATA}

T'o delete data from any of the eviluated libraries, an input file is required with two sets of three records for each deletion. Any number of deletions may be done but for each deletion there must be deletion criteria spacified. The first record of each set (target record) specifies the limits of the range of isotopes or elements, the incident and secondary particles of interest, and a value of 1 in column 34. The second record of ach set (reaction record) specifies the limits of the range of reaction descriptors and the third record of each set (data record) gives the limits of the energy range of the independent variables for which the deletion is to be made. The specifications of the fields of the first two records can be found in Table II.1. For the third record, the formats and specifications are found in Table Il.2. Both deletions and insertions of new data can be done during the same run of the code.

$$
\text { II }-4
$$




\section{Reeond 1}

\begin{tabular}{|c|c|c|c|}
\hline $1-6$ & 16 & 7.A & $1000 Z+A$ \\
\hline $8-9$ & 12 & $\mathbf{Y}_{\mathbf{i}}^{\mathbf{a}}$ & Incident particle descriptor \\
\hline $11-12$ & 12 & $\mathbf{Y}_{\mathbf{0}}^{\mathbf{a}}$ & Outgoing particle deseriotor \\
\hline $14-24$ & E11.4 & $A^{b}$ & Atomie mass for this ZA (amu) \\
\hline $26-31$ & I6 & DATE ${ }^{\mathbf{b}}$ & Curre date. (YYMMDD) \\
\hline $33-34$ & 12 & FLAG & $\begin{array}{l}\text { Designates the type of operation: } \\
\begin{array}{l}1=\text { delete, } 2 \text { = insert, } 3 \text { = retrieve, } \\
4=\text { insert (using transmission format), } \\
5=\text { retrieval (in transmission format). }\end{array}\end{array}$ \\
\hline $36-46$ & E11.4 & & Level energy of the target (MeV) \\
\hline $48-58$ & E]1.4 & & Helf-life of the target ${ }^{b}$ (sec) \\
\hline
\end{tabular}

\section{Record 2}

$1-2$

12

$\mathbf{C}^{\mathrm{a}}$

Reaction number

$3-5$

13

$I^{\text {A }}$

Reaction property designator

$6-8$

13

$\mathrm{s}^{\mathrm{a}}$

Reaction modifier flag

$10-20$

E11.4 Q0 ${ }^{\mathrm{Q}}$

Mass difference $Q$ for the reaction ( $M e V$ )

$22-32$

E11.4 $\mathrm{X}_{1}^{a}$

Value depends upon the value of $S$

$34-44$

E11.4 $\mathrm{X}_{2}^{\mathrm{a}}$

Value depends upon the value of $S$

$46-56$

E11.4 $\mathrm{X}_{3}^{\mathrm{a}}$

Value depends upon the value of $S$

a A more complete definition of these fields can be found in Tables I-A.I to I-A.4 of Appendix I-A.

$b$ Does not need to be specified for retrieval or delete. Half-life of the target must be specified only when the material is entered for the first time. 

recond.a

\begin{tabular}{|c|c|c|c|c|c|}
\hline \multirow{3}{*}{$\begin{array}{l}\text { Reartinn Pmperty } \\
\text { Integrated cross sections }\end{array}$} & \multirow{3}{*}{1} & \multicolumn{4}{|c|}{ Date stored in flelds } \\
\hline & & $\bar{T}$ & 2 & 3 & 4 \\
\hline & & $\mathbf{E}$ & $\alpha(E)$ & - & - \\
\hline Angular distributions & 1 & $\mathbf{E}$ & $\boldsymbol{u}$ & $P\left(E_{i}, W\right)$ & - \\
\hline $\begin{array}{l}\text { Energy-angle correlated } \\
\text { data }\end{array}$ & 3 & $\mathbf{E}$ & $\boldsymbol{u}$ & $E^{\prime}$ & $P\left(E_{\text {भ丩 }}\right.$, EN) \\
\hline $\begin{array}{l}\text { Energy-angle Legendre } \\
\text { coefficients }\end{array}$ & 4 & $\mathbf{E}$ & $\mathbf{E}^{\prime}$ & 2 & $\pi^{l}\left(E+E^{\prime}\right)$ \\
\hline Nu-bar & 7 & $\mathrm{E}$ & $v(E)$ & - & - \\
\hline $\begin{array}{l}\text { Histogram energy } \\
\text { distribution }\end{array}$ & 8 & $\mathbf{E}$ & $E_{1}^{\prime}$ & $\int_{E_{1}^{\prime}}^{2} P\left(E^{\prime}\right) d E^{\prime}$ & - \\
\hline Gamma-ray multiplicity & 9 & $\mathrm{E}$ & $M(E)$ & - & - \\
\hline $\begin{array}{l}\text { Total average energy of } \\
\text { particle }\end{array}$ & 10 & $\mathrm{E}$ & $E^{\prime}\left(y_{0}, E\right)$ & - & - \\
\hline A verage energy of residual & 11 & $\mathrm{E}$ & $E^{\prime}(R, E)$ & - & - \\
\hline $\begin{array}{l}\text { Maxwell-averaged } \\
\text { reaction rates }\end{array}$ & 80 & $\mathrm{kT}$ & $o v(k T)$ & - & - \\
\hline In-flight cross sections & 81 & $\mathrm{kT}$ & $\mathrm{E}$ & $\sigma(k T, E)$ & - \\
\hline $\begin{array}{l}\text { Maxwell-averaged energy } \\
\text { distributions }\end{array}$ & 84 & $\mathrm{kT}$ & $E^{\prime}$ & $\mathrm{P}\left(\mathrm{kT}, \mathrm{E}^{\prime}\right)$ & - \\
\hline
\end{tabular}




\begin{tabular}{|c|c|c|c|c|c|}
\hline \multirow[b]{2}{*}{ Resction Property } & \multirow[b]{2}{*}{$\mathbf{I}$} & \multicolumn{4}{|c|}{ Data stored in frelds } \\
\hline & & $\bar{T}$ & 2 & 3 & 4 \\
\hline $\begin{array}{l}\text { Maxwell-averaged photon } \\
\text { as particle muitiplicity }\end{array}$ & 89 & $\mathbf{k T}$ & $M(k T)$ & - & - \\
\hline $\begin{array}{l}\text { Maxwell-averaged total } \\
\text { average energy of secondary } \\
\text { particle }\end{array}$ & $9 v$ & $x T$ & $E\left(y_{0}, k T\right)$ & - & - \\
\hline $\begin{array}{l}\text { Maxwc.i-averaged average } \\
\text { energy of residual nucleus }\end{array}$ & 91 & $\mathrm{kT}$ & $\bar{E}(R, k T)$ & - & - \\
\hline $\begin{array}{l}\text { Maxwell-averaged total } \\
\text { average energy of reacting } \\
\text { particles }\end{array}$ & 92 & $\mathrm{kT}$ & $\overline{\mathbf{E}}\left(\mathbf{v}_{\mathbf{i}}, \mathrm{kT}\right)$ & - & - \\
\hline
\end{tabular}

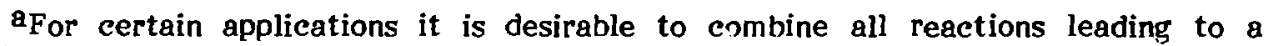
specific residual nueleus. Provision for such "activation" eross sections is made. For example, the $\left(n, n^{\prime} d\right)$ and $(n, t)$ reactions botll lead to the same residual nucleus, and the sum of the cross sections for these reactions may be entered as with the following exampie: $70 \mathrm{Zn}\left(n, n^{\prime} \mathrm{d}+\mathrm{n}, \mathrm{t}\right)^{68} \mathrm{Cu}$ to the ground state with a half-life of 32 sec.

Incident particle:

Reaction property:

Reaction type:

Outgoing particle whose

property is recorded:

Reaction modifier:

$$
\mathrm{S}=05 \text { (activation) }
$$$$
\mathrm{S}=05 \text { (activation) }
$$
particle is given)

$$
\begin{aligned}
& y_{i}=01 \text { (neutron: } \\
& I=00 \text { (integrated cross zetion) } \\
& C=65 \text { (activition) }
\end{aligned}
$$

$y_{0}=00$ (not applicable since no property of a secondary

$w_{2}: \quad w_{2}=0 .\left(x_{2}\right.$ field $)$

$\tau_{2}: \quad \tau_{2}=32 .\left(x_{3}\right.$ field $)$

This gives for the reaction code

$\begin{array}{lllllllll}y_{j} & 1 & C & y_{j} & s & Q_{0} & x_{1} & x_{2} & x_{3} \\ 01 & 00 & (65) & (00) & 05 & 29068 . & 0 . & 32 . & \end{array}$




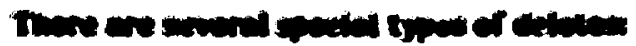

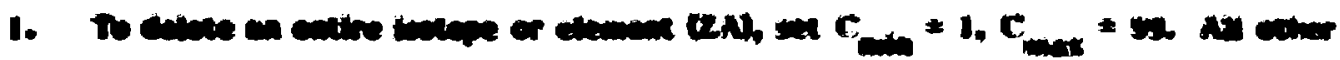

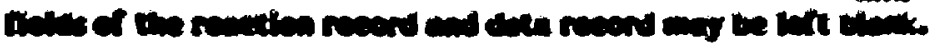

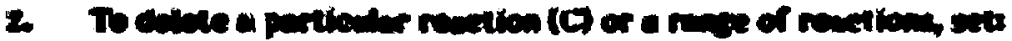

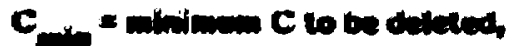

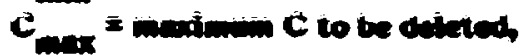 \\ $\min =\omega_{0}$ \\ $\operatorname{tmax}_{\max }=\%$ \\ All other field must be set to the minimum-maximum renge decired. \\ 3. To dalete all of a partieular resetion property defignator ( 0 , leave both cata records blenk. All other felds must be set to the minimum maximum range desired. \\ If the limits speciffed by the delete request eannot be satiafied, a comment is printed and the code continues. The data sets representing deletes and inserts may be in any order in the input file. The code sorts the sets, performs the delete operations and then inserts new data one target at a time.}

\section{INSERTION OF DATA}

If data are to be inserted into the library, the first two records (the tanget and reaction records) must be as deseribed in Table 11.1. These records determine the point in the library at which insertions are to be made. The third and following records are data records containing the data to be inserted. They are specified with respect to the format and content described in Table II.2 if the flag in column 34 of the first record is "2n. If the flag in column 34 of the first record is " 4 ", the format and content of the data records are as specified in Tables I-A.6, I-A.7 and I-A.8 of Appendix I-A. Following the last record of each data set to be inserted is a record wiih the number 1 in column $\mathbf{7 2}$. There is no limit to the number of data records, but angular distributions may have no more than 201 entries for each incident energy. Whenever appropriate, data are merged with existing data, but if equal points are encountered in the merge, they are printed out with the original data left in the library. The code then proceeds. If a match cannot be made between the specifications stated on the first two records of a set to be inserted and those found on the evaluated library, a new isotope (ZA), a new reaction $(C)$, a new reaction modifier $(S)$, any of the $X_{i}$ fields, or a new reaction property designator (l) is created to fulfill the required specifications. Insert requests are read by the code until it senses an end of file, which signals the code to begin processing the requests.

There is no limit to the number of delete and insert requests that can be present.

$$
\text { II }-8
$$




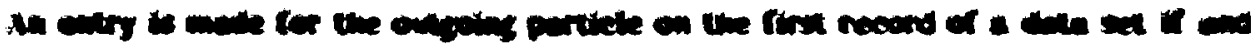

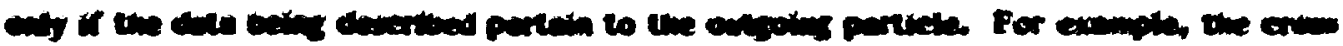

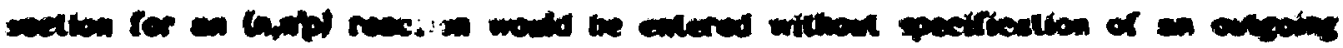

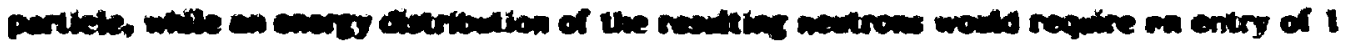

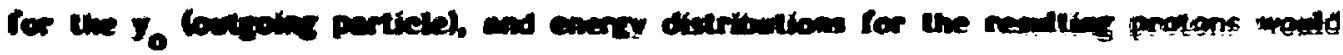

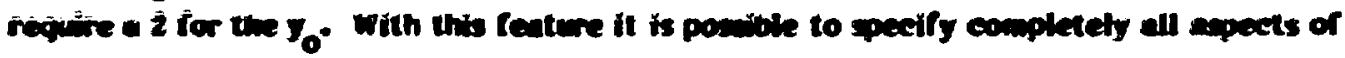
reactiona huving more thas one type of emergent partlele.

\section{E MAKING A NEW LBRAKY ab inflio}

In the special ease of making an entirely new library file, the entry in the calling sequence for (library name) should be "none". In such a ease the file used for input requires a first record with the following information:

\begin{tabular}{|c|c|c|}
\hline Columns & \multicolumn{2}{|l|}{ Significance } \\
\hline $1-6$ & \multicolumn{2}{|l|}{ Date (Y YMMDD) } \\
\hline 7-8 & \multicolumn{2}{|c|}{ Institution ( 1 for LLN $L, 2$ for AWRE, 3 for ENUF/B) } \\
\hline $9-10$ & \multicolumn{2}{|l|}{ Year } \\
\hline $11-12$ & \multicolumn{2}{|c|}{ Edition of the library } \\
\hline $13-14$ & \multicolumn{2}{|c|}{ Version of the edition entered in columns $11-12$} \\
\hline \multirow[t]{5}{*}{15} & Type of library: & $1=$ neutron interaction \\
\hline & & $2=$ photon interaction with cold atoms \\
\hline & & $3=$ photon interaction with hot media \\
\hline & & $4=$ charged particle interaction \\
\hline & & $5=$ neutron-induced activation \\
\hline
\end{tabular}

Making such a new library rather than updating an existing library is a rare occasion.

\section{F. OUTPUT}

There are several different forms of printout from the code which tell the user what has been done. This, of course, is in addition to any files that have been made. The order of the output is as follows:

1. The evaluated library identification word from the input library is decoded and printed with appropriate labels deseribing the meaning of each quantity. 'This is followed by the date for this library.

2. The delete and insert requests or the retrieve requests are printed as they were read from the input cards.

3. A printout is made showing the computer time used to process this update.

$$
\mathrm{II}-9 / \ddot{1}-10
$$




\section{ChAPTER un.}

ENDEP AND AUTODEP

$$
\text { III }-1 / \ddot{11}-2
$$




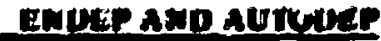

\section{A. In rmovertion}

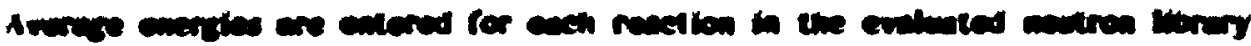

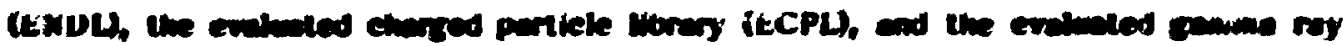

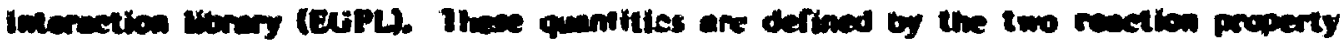

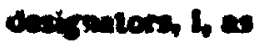

I 10 the total everuge energy in each outgoing particle field, ISyaste,

I = II the averuge energy of the residual nucleas.

See Appendix I-A for the definitlons of resctions and resetion property denignators. Giamma ray peetra may or may not be present in the evaluated library for a given nuclear reaction. If the spectral data are not present, then the gamma rays from the reaction are included within the gamma ray production $(c=55)$ data. In either case, the I = 10 data for the resultant gamma field are calculated by the code. Additionally, all nuclear reactions have a residual or final state nucleus. If this nucleus is either a neutron or a light charged particle, it is tagged by an outgoing particle designator, $11 \leq y_{0} \leq 16$ and only $I=10$ data are calculated. If the residual's $\mathrm{ZA}>2004$, then $\mathrm{I}=11$ data are also calculated.

ENUEP calculates the required average energies from the existing data, e.g., secondary particle spectra and multiplicities. The latter may be implicit in the reaction as with the $n, 2 n$ reaction where the implied neutron multiplicity is 2 or explicit as for gamma-rays from capture where energy dependent multiplieities are given in the library. If $1=3$ double differential data are present for the reaction, no ENDEP calculation is performed and the existing $I=10$ and 11 data are utilized. AUTODEP is the automatic version of ENDEP. When any changes are made in the evaluated library for a given reaction pertaining to energy deposition, e.g., changes to $I=1,4,7-9$ data, AUTODEP is initialized by the UPDATE code for the isotope in question. The new energy deposition terms are then automatically included in the update of the library. It is assumed that the target nucleus is at rest. Specifically as a function of reaction designator, $C$, the following energy deposits are calculated:

$8 \leq \mathrm{C} \leq 49 \quad \mathrm{I}=10$ and 11 data unless, as above, the residual nucleus has $\mathrm{ZA} \leq$ 2004. In the latter case only $I=10$ data are calculated. The particle multiplicity is included in the $I=10$ data. 


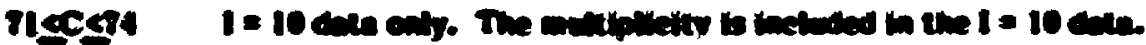

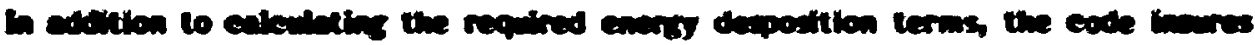

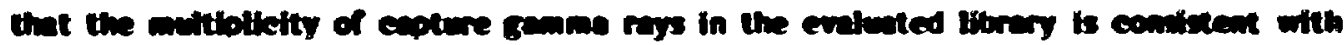

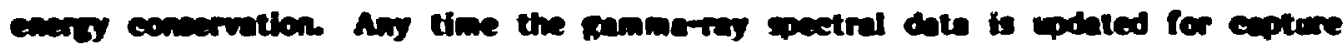

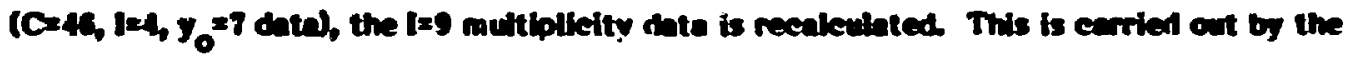
we of Bq, (ILC-1) of Appendix II.C, in confonction with Eq (IIL2). The enersy deposition terms are then ealeulated as deweribed in this ehopter.

Two general elswes of models are used to enleulate the required averange energies for a given reaction. These are the $E$ models and the residual models. The method of caleulating the average enengy in an outgoing particle or gamma-ray fiek is baser on the type of data in the file for the particle in question, e.g., two-body center of mass angular distribution, particle speetra, ete. This is specified by the $\bar{E}$ model. The average energy into the gamma ray field and the average energy of the residual mueleus is determined both by the $\overline{\mathrm{E}}$ model and by the reaction $\mathrm{C}$ number. These specify the residual model.

Both the $\overline{\mathrm{E}}$ models and the residual models will be defined in the next section in terms of the specific kinds of information that are characteristic of the evaluated data libraries. This will be followed by specific descriptors for each $\bar{E}$ model and then for each residual model. Finally, the required code input, operation, and output will be given.

Several comments should be made at this time regarding energy conservation and balance. If the average energy or the gamma ray field $\left(I=10, y_{0}=7\right)$ is calculated to be negative when no explicit photon spectra are entered for that reaction, it is set to zero and the calculation then continues, with an error message printed in the output. An overall reaction energy balance is conserved by this approach. If the calculated average energy of the residual nucleus ( $I=11$ data) is also negative, it is set to zero and energy is no longer conserved. Again, an error message is printed. The basic evaluated data must be revised in these cases since too much energy has been put into the evaluated secondary particle spectra. The resultant iterative procedure, when the conservation criteria are satisfied, yields an improved set of data.

\section{B. $\bar{E}$ AND RESIDUAL MODELS}

The reaction type designator, $C$, reaction modifier, $s$, and the reaction property designator, 1 , determine the $\overline{\mathrm{E}}$ model for a given outgoing particle, and the model to be

$$
\text { III - } 4
$$




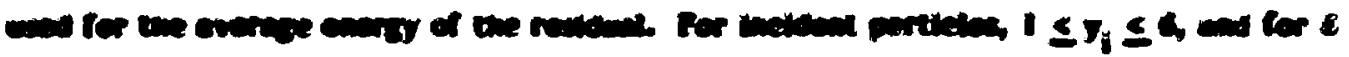

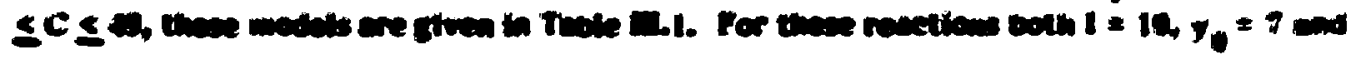

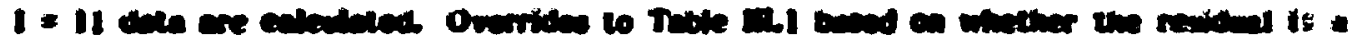

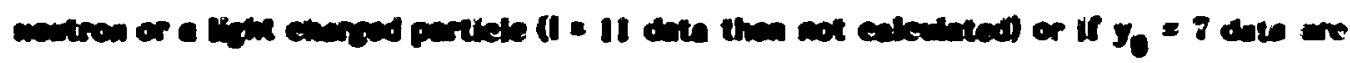

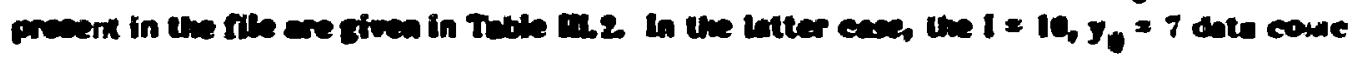

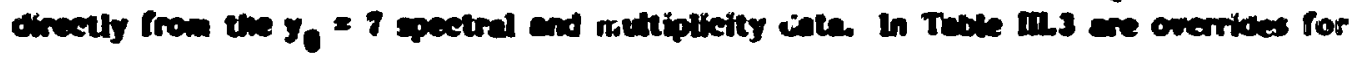

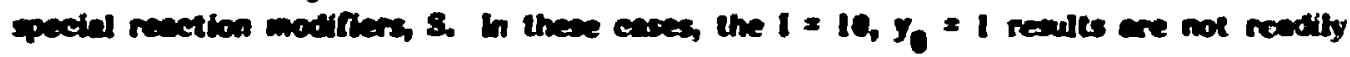
cbtainable fron the fle information and are obtained from eneny conservation. These models have been used for the ${ }_{1}^{2} \mathrm{H}$ and ${ }_{4}^{9} \mathrm{Be}(n, 2 n)$ reaction.

TABLE II.. E. E and residual models for $1 \leq y_{i} \leq 6$, and no $y_{0}>10$ (no residual nucleus being either a neutron or a changed particie), or yo $=7$ (no secondary gamma ray data) present.

\begin{tabular}{cllll}
\hline C & S & $I^{c}$ & $\bar{E}$ model & Residual model \\
\hline $8-10$ & 0 & 1 & A & 1 \\
11 & 1 & 1 & A & 1 \\
11 & 0 & 4 & D & 2 \\
12 & 0 & 4 & D & 38 or $2 a^{b}$ \\
$13-14$ & 0 & 4 & D & $2 a$ \\
$20-22,24-26$ & 0 & $M a n y$ & E & 4 \\
$20-22,24-26$ & 1 & 4 & $D$ & 3 \\
$40-42,44,45$ & 1 & $1 \& 4^{\mathrm{a}}$ & $\mathrm{C}$ & 5 \\
$40-42,44,45$ & 0 & 1 & A & 1 \\
\hline
\end{tabular}

a $I=I$ is for the first particle (a neutron in all cases but $C=21$ ); $I=4$ is for the second particle.

b Both models are programmed into the code, reaction type $2 \mathrm{a}$ is currently being used.

c $I=1$ and 4 may also be given. This is an uncorrelated energy-angle reaction and only the $I=4$ data are used. 


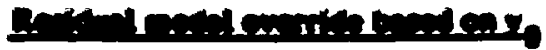
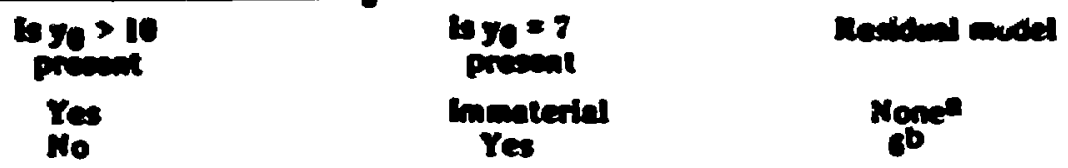

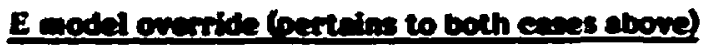

$\begin{array}{lcl}y_{0} & 1 & E \text { model } \\ =7 & 1 \& 9^{e} & D^{e} \\ \neq 7 & 1 & A \\ \neq 7 & 4 \& 9 & D^{d}\end{array}$

TABLE ill.3. Overrides to Table 1 when special reaction modifiers, $S$, are present for a given $\mathrm{C}$.

\begin{tabular}{cccccc}
\hline $\mathrm{S}$ & $\mathbf{y}_{\mathrm{i}}$ & $\mathrm{C}$ & $\overline{\mathrm{E}}$ model & Residual model \\
\hline $\mathbf{8}$ & 1 & 12 & $\mathrm{~F}$ & None \\
10,11 & 1 & 12 & $\mathrm{~F}$ & None \\
\hline
\end{tabular}

The $\overline{\mathrm{E}}$ and residual models for production cross sections are given in Table III.4. The only values calculated for these reactions are the $I=10$ data for the secondary particies. These results are used in the GAMCHK code (see Chapter IV) that compares the $\sigma \cdot \overline{\mathrm{E}}$ values (barn $\mathrm{MeV}$ ) for the gamma ray and electron production cross sections versus the sum of the $\sigma \cdot \bar{E}$ values for $1=10, y_{0}=7$ and 9 data for the particle interaction reactions, $11 \leq \mathrm{C} \leq 49$. In principle, these results should be the same; in practice they of ten are not, and changes to one or the other types of information has to be made. 


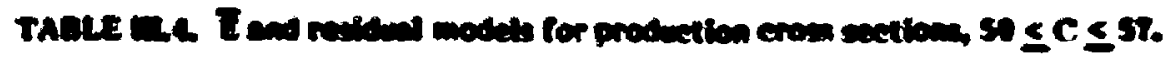

\begin{tabular}{|c|c|c|c|c|}
\hline c & $\mathbf{s}$ & $t$ & E model & Revilumal modet \\
\hline $\begin{array}{l}s e-57 \\
s 0-57\end{array}$ & : & 1 & $\begin{array}{l}61 \\
02\end{array}$ & $\begin{array}{l}\text { None } \\
\text { Mome }\end{array}$ \\
\hline
\end{tabular}

Finally, the E and residual madels for gamma ray induced reactions, $y_{i}=7$, are shown in Table Im.5. The basic assumption is that all enenty is deposited locally, lie, there are only $1=10$ data caleulated for $y_{0}=7$ and hence no residual values are calculated.

TABLE III.5. E and residual models for $y_{i}=7 ; 71 \leq C \leq 74$, gamma ray induced reactions.

\begin{tabular}{cccc}
\hline $\mathrm{C}$ & $1^{\mathrm{a}}$ & $\overline{\text { E model }}$ & Residual model \\
\hline 71 & $\mathrm{O}$ & $\mathrm{H1}$ & $7 \mathrm{a}$ \\
72 & 4 & $\mathrm{H} 2$ & $7 \mathrm{~b}$ \\
73 & $\mathrm{O}$ & $\mathrm{H} 3$ & $7 \mathrm{c}$ \\
74 & 4 and 9 & $\mathrm{H} 4$ & $7 \mathrm{~b}$ \\
\hline
\end{tabular}

Ignore all other $\mathbf{1}=0$ data present for the regction.

C. E MODEL DESCRIPTIONS

The specifications for each $E$ type designater are as follows:

E Model A-Diserete two-body

Consider particle 1 interacting with target 2 yielding particle $\mathrm{x}$ and the residual $\mathrm{y}$. We obtain the average energy for particle $x, \overline{E_{x}\left(E_{1}\right)}$, from Eq. (III-A.12) of Appendix III-A.

$$
\overline{E_{x}\left(E_{1}\right)}=a_{1 x} E_{1}+a_{2 y}\left(E_{1}-E_{l_{\alpha}}\right)+2\left[a_{1 x} a_{2 y} E_{1}\left(E_{1}-E_{l_{\alpha}}\right)\right]{ }^{l / 2} g_{x_{1}}\left(E_{1}\right),
$$




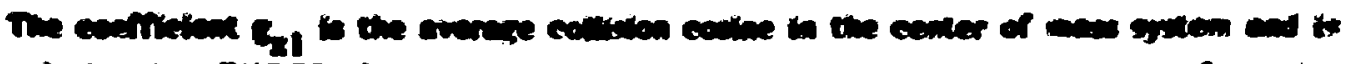

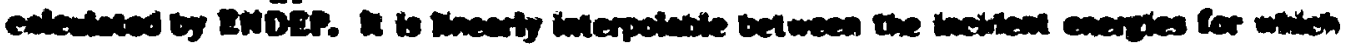

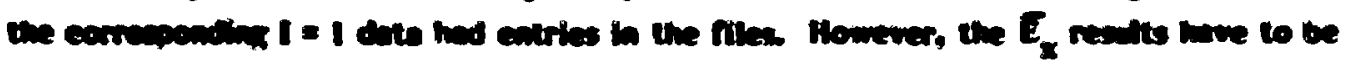

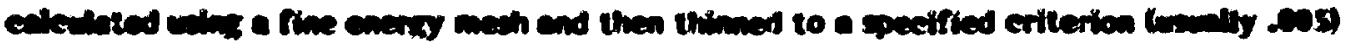

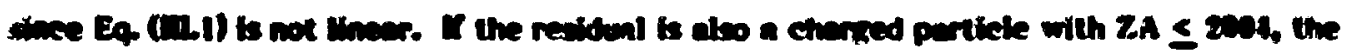
procedure to repeated for it.

\section{E Modet B - Continumen two-bodv}

The average enersy of all outzoing particles of type $x_{0} \bar{E}_{x}$, is

$$
\overline{E_{x}\left(E_{1}\right)}=m_{x}\left(E_{1}\right) \int_{E_{x_{\min }}}^{E_{x_{\max }}} E_{x \pi_{0}}\left(E_{1}+E_{x}\right) d E_{x} \text {, }
$$

where $\pi_{0}\left(E_{1}+E_{x}\right)$ are the $I=4$ data $\left(l=0\right.$ only) and $m_{x}$ is the particle multiplieity. Eq. (III.2) is integrated analytically sinee $\pi_{0}\left(E_{1}+E_{x}\right)$ are linearly interpolable in $E_{x} \cdot \bar{E}_{x}$ is calculated at the merged energies in the $I=4$ data and the multiplicity sets.

The multiplicities are unity except for those noted in Table III.6, and for the following cases for which $m_{x}$ is energy dependent. For $c=15$ and $46, m_{x}$ for $y_{0}=7$ is given by $\mathrm{I}=9$ (multiplicity) data. For $\mathrm{C}=15, \mathrm{~m}_{x}$ for $\mathrm{v}_{0}=1$ is given by $\mathrm{I}=7(\vec{v})$ data.

TABLE III.6. Constant values for particle multiplicity greater than unity for specific C numbers and $\mathbf{y}_{0}$.

\begin{tabular}{lll}
\hline $\mathrm{c}$ & $y_{0}$ & $\mathrm{~m}_{\mathbf{x}}$ \\
\hline 12 & 1 & 2 \\
13 & 1 & 3 \\
14 & 1 & 4 \\
27 & 6 & 2 \\
29 & 1 & 2 \\
32 & 1 & 2 \\
37 & 6 & 2 \\
\hline
\end{tabular}




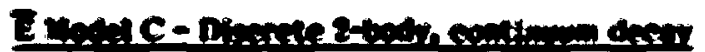

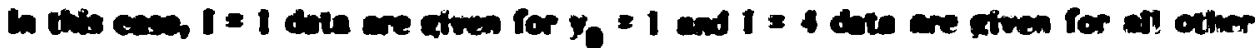

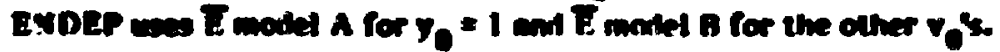

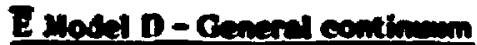

$1=4$ and 9 date cre gfiven $s 0$ that ExIJEP uses $E$ modet f. Note that hoth $\nabla_{n}=7$ and $y_{0}=9$ cutput ean eome from ratiative capture.

If the $1=4$ and 9 ata for $y_{0}=7$ or 9 do not extend to the maximum eneryy (eurrentw set at 20 MeV, the missing data have been preprocessed and are included in the particle production information $(C=55$ and 57 . We therefore must provide information for $I=10$ at 20 MeV from Eq. (IIIC.6) of Apsendix IIIC for $y_{0}=$,

$$
\overline{E_{x}\left(E_{1}\right)}=\frac{M_{2}}{M_{1}+M_{2}} E_{1}+Q,
$$

where $Q$ is the reaction $Q$ value, $M_{2}$ is the target mass, and $M_{1}$ is the mass of the incident particle. If $y_{0}=9$ data are also present, we set $\bar{E}_{x}\left(E_{1}\right)=0$, for $y_{0}=9$ and $E_{1}=$ $20 \mathrm{MeV}$.

\section{$\bar{E}$ Model E - Fission}

For $y_{0}=1, I=4$ and 7 data are given and $E$ model $B$ is used. Similarly for $y_{0}=7, I=$ 4 and 9 data are given and $E$ model $B$ is used.

$\bar{E}$ Model $F-C=12, S=8$ or 10,11 for $\mathrm{y}_{\mathrm{i}}=1$

In either case, for all $y_{0} \neq 1, \bar{E}$ model $B$ is user. For $y_{n}=1, \bar{E}_{x}$ cannot be easily determined from the data so energy conservation is used. This results in, for $y_{0}=1$,

$$
\bar{E}_{x}=E_{1}+Q-\sum_{y_{0} \neq 1} \bar{E}_{y_{0}}
$$

where $Q=$ the mass difference $Q_{0}$ value for the multibody breakup to the final end products. For $\mathrm{ZA}=1002, \mathrm{Q}=-2.23 \mathrm{MeV}$ and for $\mathrm{ZA}=4009, \mathrm{Q}=-1.573 \mathrm{MeV}$.

\section{E Model G - Production cross sections}

Submodel Gl. For the given $y_{0}, \bar{E}$ model $D$ is used with inultiplicity, $m_{x}=1$.

Submodel G2. The incident energies come from the $I=0$ data. Then for $y_{0}=7, \bar{E}_{\mathrm{y} 0}$ $=\mathrm{Xl}$, the value entered in the $\mathrm{Xl}$ field. 


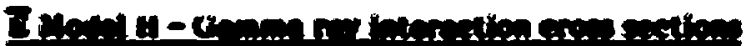

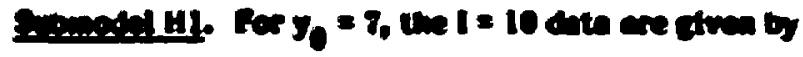

$$
E_{\mathbf{y}_{0}}=\mathbf{E} \text {. }
$$

where E ts the incident particie enery.

Sebodel H2. Vior $y_{0}=7, E$ model $B$ is uned with the multiplieity, $m_{x}=1$.

Submodel H3. No $1=10$ date are caleulated for the reaction.

Submodel H4, For $y_{0}=7, E$ model $B$ is used with a multiplieity, $m_{x}=2$.

\section{RESIDUAL MODELS}

The description of each model used to calculate the average energy of residuals will now be given. For convenjence, the nomenclature used here will be different from that used previously in this chapter. Let $y=$ particle, $A=$ nucleus, $Q_{j}=$ the mass difference $Q$ value, $\bar{E}_{Y}=$ the $I=10, y_{0}=7$ value, and $\bar{E}_{R}=$ the $1=11$ data. Subscripts 0 refer to the projectile and target, 1 to the two products from the two body reaction, and 2, etc., to resultant pairs of products from subsequent decay events. Two-body kinematics are discussed in Appendix III-A and decay kinematics in Appendix II-B, with those results being used here as needed.

Residual Model 1 - Discrete two-body

$$
(\mathrm{y}_{0}+\mathrm{A}_{0}-\overbrace{\mathrm{A}_{1}} \mathrm{Q}_{0,1}=\left[\left(\mathrm{M}_{\mathrm{y}_{0}}+\mathrm{M}_{\mathrm{A}_{0}}\right)-\left(\mathrm{Mi}_{\mathrm{y}_{1}}+\mathrm{M}_{\mathrm{A}_{1}}\right)\right] \mathrm{c}^{2}
$$

Given: $M_{y_{0}} M_{A} M_{0} M_{1} Q_{0_{0,1}}$ (reaction $Q_{0}$ ), $W_{A_{1}}^{*}$ (XI field), $E_{y_{0}}, \bar{E}_{y_{1}}$. Calculate: (See Appendix III-A)

$$
\begin{aligned}
& \bar{E}_{\gamma}=W_{A_{1}}^{*}, \\
& Q_{0,1}=Q_{0,1}-W_{A_{1}}^{*}, \\
& \bar{E}_{R}=\bar{E}_{A_{1}}=E_{y_{0}}+Q_{0,1}-\bar{E}_{y_{1}} .
\end{aligned}
$$




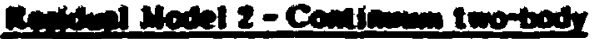

\section{(3) (ब) (1)}

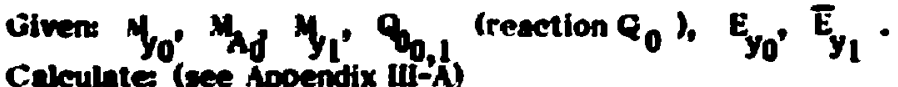

$$
\begin{aligned}
& M_{A_{1}}=M_{y_{0}}+M_{A_{0}}-M_{A_{1}}, \\
& \bar{W}_{A_{1}}=Q_{0,1}+\frac{\left(M_{y_{0}} M_{y_{1}}+M_{A_{0}} M_{A_{1}}\right)}{M_{A_{1}}\left(M_{y_{0}}+M_{A_{0}}\right.}-\frac{M_{y_{0}}+M_{A_{0}}}{M_{A}} \bar{E}_{y_{1}}, \\
& \bar{E}_{Y}=\bar{W}_{A_{1}} * \\
& Q_{0,1}=Q_{0},-\bar{W}_{A_{1}}^{*}, \\
& \bar{E}_{R}=\bar{E}_{A_{1}}=E_{y_{0}}+Q_{0,1}-\bar{E}_{y_{1}} .
\end{aligned}
$$

\section{Submodel 2a}

Let $m=$ multiplicity of $y_{1}(m=2, C=12 ; m=3, C=13 ; m=4, C=14)$. Then let

$$
\begin{aligned}
& \mathrm{in}_{\mathrm{y}_{1}}=\mathrm{mM}\left(\mathrm{y}_{1}\right), \\
& \bar{E}_{\mathrm{y}_{1}}=\bar{E}_{\mathrm{mn}} .
\end{aligned}
$$

where $\bar{E}_{\mathrm{mn}}$ is the $\mathrm{I}=10$ value calculated from the ENDL (or ECPL) $1=4$ data as defined by $\overline{\mathrm{E}}$ model B. Repeat Eqs. (1II.9) - (III.13) above using the results of Eqs. (1I.14) and (III.15). 
Note that this tusction is very similer to residual model 5.

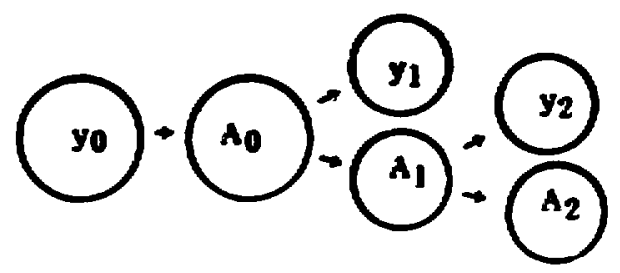

Given: $M_{0}, M_{A_{d}} M_{y_{i}}, M_{y_{2}}, Q_{0_{0,2}}$ (reaction $\left.Q_{0}\right), E_{y_{0}}, \bar{E}_{y_{1}}, \bar{E}_{y_{2}}$. Calculate: (sce Appendices III-A and III-B)

$$
Z A\left(A_{1}\right)=Z A\left(y_{0}\right)+Z A\left(A_{0}\right)-Z A\left(y_{1}\right)
$$

Obtain $M_{A_{1}}$ from mass tables $M_{A_{1}}=M\left(Z A\left(A_{1}\right)\right)$

$$
Q_{0,1}=\left[\left(M_{y_{0}}+M_{A_{0}}\right)-\left(M_{y_{1}}+M_{A_{1}}\right)\right] e^{2}
$$

Redefine ${ }^{\mathrm{M}} \mathrm{A}_{1}$ as per Eq. (III.9),

$$
\bar{W}_{A_{1}}^{*} \text { from Eq. (III.10), }
$$

$\mathrm{Q}_{0,1}$ from Eq. (1II.12),

$$
\bar{E}_{A_{1}}=E_{y_{0}}+Q_{0,1}-\bar{E}_{y_{I}} \text {, }
$$

$\mathrm{M}_{\mathrm{A}_{2}}=\mathrm{M}_{\mathrm{A}_{\mathrm{I}}}-\mathrm{M}_{\mathrm{y}_{2}}$,

$\mathrm{Q}_{1,2}=\mathrm{Q}_{0,2}-\mathrm{Q}_{0,1}$,

$\overline{\mathrm{W}}_{\mathrm{A}_{2}}^{*}=\mathrm{Q}_{0,2}+\overline{\mathrm{W}}_{\mathrm{A}}^{*}+\frac{\mathrm{M}_{\mathrm{y}_{2}}}{\mathrm{M}_{\mathrm{A}_{2}}} \overline{\mathrm{E}}_{\mathrm{A}_{1}}-\frac{\mathrm{M}_{\mathrm{A}_{1}}}{\mathrm{M}_{\mathrm{A}_{2}}} \overline{\mathrm{E}}_{\mathrm{y}_{2}}$, 


$$
\begin{aligned}
& F_{Y}=\bar{E}_{A_{2}}^{*}, \\
& E_{U}=E_{A_{2}}=E_{y_{0}}+Q_{O_{0,2}}-E_{y_{1}}-E_{y_{2}}-E_{Y} .
\end{aligned}
$$

(III.27)

\section{Submidel 3a -}

$$
\begin{aligned}
& y_{1}=y_{2} \\
& \bar{E}_{y_{1}}=\bar{E}_{y_{2}}=\frac{\bar{E}_{2 n}}{2}
\end{aligned}
$$

where $\bar{E}_{21}$ is the $i=10$ value calculated from the ENDL (or ECPL) $I=4$ data as defined

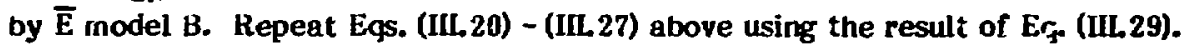

\section{Residual Model 4 - Fission}

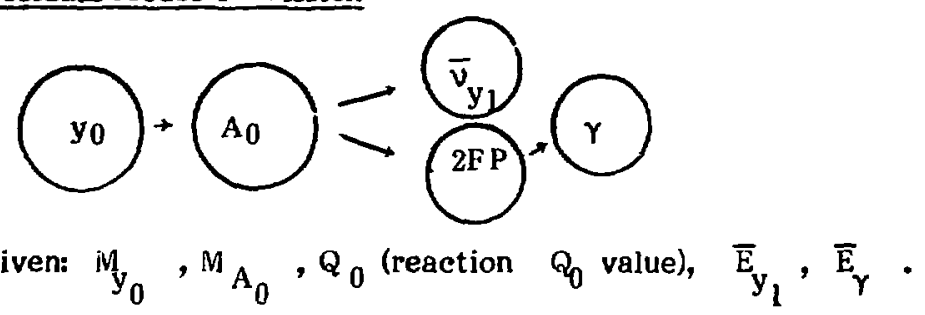

Calculate:

$$
\bar{E}_{R}=\bar{E}_{2 F P}=E_{y_{0}}+Q_{0}-\bar{E}_{y_{1}}-\bar{E}_{\gamma} \text {. }
$$

\section{Residual Model 5 - Discrete two-body, continuum decay}

Note that this reaction is very similar to residual model 3.

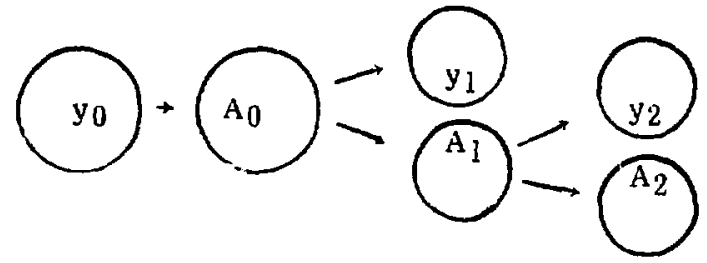




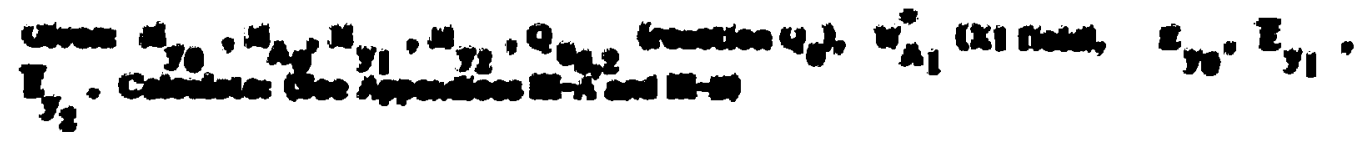

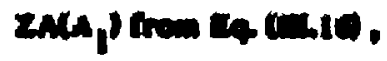

(a)

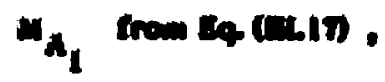

(it)

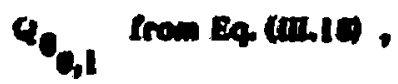

(ain)

$Q_{0,1}$ Irom Bq (I,n)

(III.3)

$E_{A_{1}}$ from Eg (III) ,

(III35)

$M_{A_{2}}$ from Eq (IIL23),

(II.36)

$Q_{0_{1,2}}$ from Eq (ILL24)

$\bar{W}_{A_{2}} *$ from Eg. (1L.25)

(II. 38)

$\bar{E}_{Y}$ from Eq. (1IL.26)

$\bar{E}_{R}=E_{A_{2}}$ from Eq. (II.27),

(III.40)

Residual Model 6 - Radiative capture

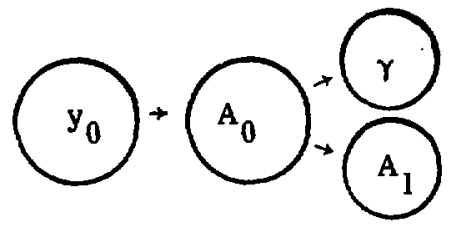

Given: $M_{y_{0}}, M_{A_{0}}, Q_{0_{0,1}}$, (reaction $Q_{0}$ ), $E_{y_{0}}, \bar{E}_{\gamma}$.

Calculate: (See Appendix IIIC) 


$$
\text { 5.5. }
$$

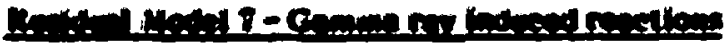

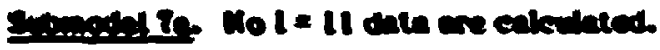

$$
\begin{aligned}
& \text { sendatio } E_{R}=E-E_{Y} \text {. } \\
& \text { more E is the ineident eneray. } \\
& \text { somodel 7e } E_{R}=E \text {. }
\end{aligned}
$$

\begin{tabular}{|c|c|c|}
\hline Colurans & Format & Remarks \\
\hline 1 & il & $\mathbf{Y}_{\mathbf{i}}$ - incident partiele designator. \\
\hline $2-5$ & $\mathbf{F} 4.3$ & $\begin{array}{l}\text { EPCK - fractional error for energy check printout. Only } \\
\text { when the calculated energy discrepency }>\text { EPCK will a } \\
\text { comment be printed on output. }\end{array}$ \\
\hline $6-11$ & i6 & N NZAB - Beginning target ZA number. \\
\hline $12-17$ & i6 & N NZAE - Ending target ZA number. \\
\hline $18-20$ & i3 & NRCTB - Beginning reaction C number. \\
\hline $21-23$ & i3 & NRCTE - Ending reaction C number. \\
\hline $24-26$ & i3 & $\begin{array}{l}\text { NUELOP }=0 \text {, make necessary } 1=10 \text { and } 11 \text { delete cards for } \\
\text { update in the output file. }=1 \text {, no delete cards will be made. }\end{array}$ \\
\hline $27-29$ & i3 & $\begin{array}{l}\text { NPNTOP-If }>0 \text {, increasing amounts of debug output are } \\
\text { given as NPNTOP increases from } 1 \text { to } 5 \text {. }\end{array}$ \\
\hline
\end{tabular}

\section{E IN PUT, OPERATION, AND UUTPUT}

The input fike for ENDEP contains the following information:

The calling sequence for ENDEP is: omega endep (input file) (box no.) (hsp option) (library name) 0 (output file name) (output file size) / TV 


\section{The

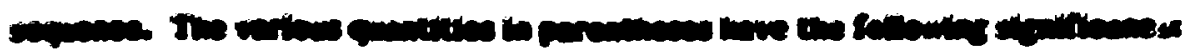

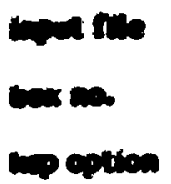

Morey name

-

output file name

output file size

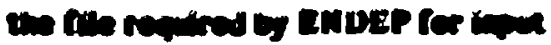

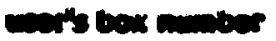

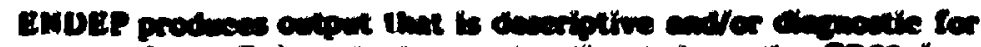

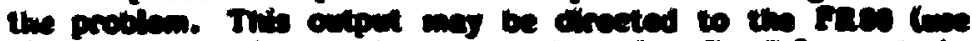

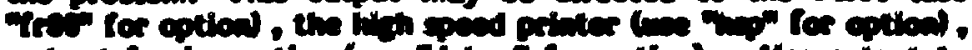

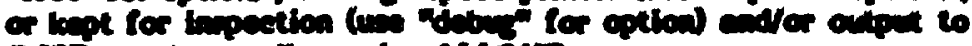

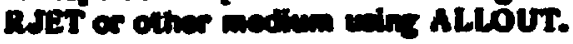

\section{The thenry file of evaluated date und by the cork.}

The zero in this position indieates that the library exists as a file. The zero must be included in the enlling sequence.

The name of the file to be created as output. This file contains the $I=10$ and 11 data.

If equal or less than 99 , it is the size of the file to be created in units of 100000 . If greater than 99 , it is the actual file size to be created. When the code finishes it will shrink the file to the size needed.

An example of running ENDEP using an input file DEPIN with a library called ND82 1201 to make an output file (with the $I=10$ and 11 data) called DEPOUT is omega endep depin T61 debug nd821201 0 depout $10 / .9 .999$

The HSP output consists of the following information:

1. The $y_{i}, C, S, Q_{0}$, and $X 1$ fields for all reactions.

2. If $\bar{E}_{\gamma}$ is calculated to be negative, the code prints out EBARG( $\left.i\right)=$ , where $i$ is the counter for the $\mathrm{I}=10, \mathrm{y}_{0}=7$ data.

3. If $\bar{E}_{R}$ is calculated to be negative, the code prints out EBARAl( $\left.i\right)=$ , where i is the counter for the $\mathrm{I}=11$ data.

4. The available energy for a reaction is $E+Q$. The Out (output) energy is all $^{\Sigma_{y_{0}}}(\mathrm{I}=10)$ $+(I=11)$. Then if |Available-Out $/$ Available > EPCK, write out ENERGY DISCREPA NCY $=$ A vailable - Out, $=\mid$ A vailable-Out $\mid * 100 /$ Available $\%$. 


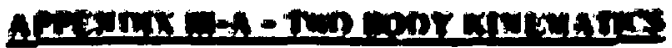

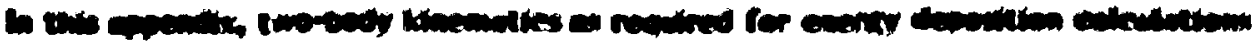

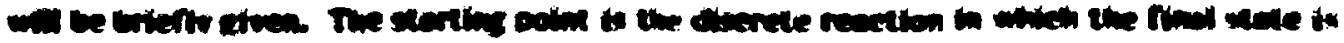

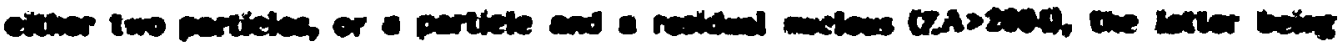

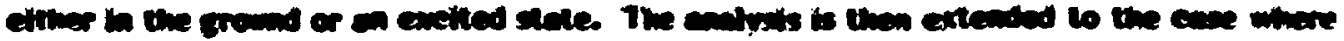
tore froml state is in the exondinmm.

In the civenete two-bods reaction, one has projectile 1, enens $E_{j}$, intercetion with

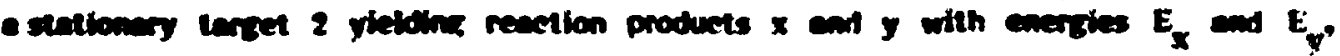
repectively. Conservation of enery in the Lborntory (L) frame yields

$$
E_{\mathbf{1}}+Q=E_{\mathbf{x}}+E_{\mathbf{y}}
$$

where the reaction $Q$ valus is given by (note that $w_{1}^{*}$ is usually zero):

$$
Q=Q_{0}+\left(w_{1}^{*}+w_{2}^{*}\right)-\left(w_{x}^{*}+w_{y}^{*}\right)
$$

and

$$
Q_{0}=\left[\left(M_{1}+M_{2}\right)-\left(M_{x}+M_{y}\right)\right] c^{2}
$$

In these expressions, $w$ refers to level excitation energy and $M$ to mass. The next step is to transform to the center of mass (CM) system, use energy and moinentum conservation, and transform back to the $L$ system to obtain for the kinetic energy of particle $x,{ }^{1,2}$

$$
E_{x}=a_{1 x} E_{1}+a_{2 y}\left(E_{1}-E_{1_{\alpha}}\right)+2\left[a_{1 x} a_{2 y} E_{1}\left(E_{1}-E_{1}\right)\right]^{1 / 2} \eta_{x}
$$

where $\mathrm{z}$ is the collision cosine of particle $\mathrm{z}$ in the CM system. The other terms in Eq. (III-A.4) are defined by

$$
\begin{aligned}
& a_{1 x}=\frac{M_{1} M_{x}}{\left(\bar{M}_{1}+M_{2}\right)\left(\bar{M}_{x}+M_{y}\right)}, \\
& a_{2 y}=\frac{M_{2} M_{y}}{\left(M_{1}+M_{2}\right)\left(M_{x}+M_{v}\right)},
\end{aligned}
$$




$$
\text { 1. }=\frac{m_{1}+k_{2}}{m_{2}} Q \text {. }
$$

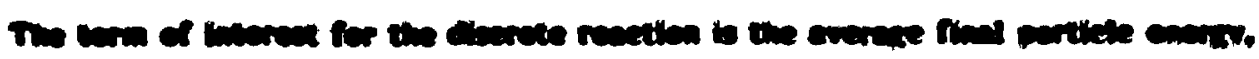

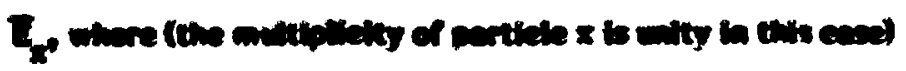

$$
E_{x}=\int_{E_{\text {min }}}^{E_{\max }} E_{x}\left(E_{1}+E_{x}\right) e_{x}
$$

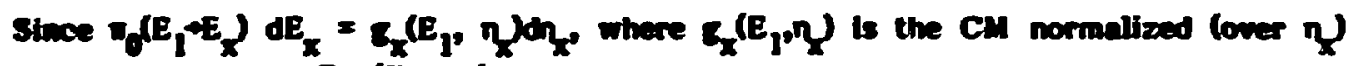
anglar disturibution, Eq (III-A.8) becomes

$$
E_{x}=\int_{-1}^{+1} E_{x}\left(E_{1}, \eta_{x}\right) g_{x}\left(E_{1}, \eta\right) d n_{x},
$$

where $E_{X}\left(E_{1}, \eta\right)$ is given by $E_{q}$ (II-A.4). Expanding the angular distribution in Legendre polynomials,

$$
E_{x}\left(E_{1}, n_{x}\right)=\sum_{\ell=0}^{\infty} \frac{2 \ell+1}{2} B_{x_{l}}\left(E_{1}\right) P_{\ell}\left(n_{x}\right),
$$

where

$$
g_{x_{\ell}}\left(E_{1}\right)=\int_{-1}^{+1} g_{x}\left(E_{1}, \eta_{x}\right) P_{\ell}\left(\eta_{x}\right) d \eta_{x} .
$$

Substituting Eq. (III-A.10) into Eq. (111-A.9) and carrying out the integration with the use of the orthagonality relation yields

$$
\bar{E}_{x}=a_{1 x} E_{1}+a_{2 y}\left(E_{1}-E_{1}\right)+2\left[a_{1 x} a_{2 y} E_{1}\left(E_{1}-E_{1}\right)\right]^{1 / 2} g_{x_{1}}\left(E_{1}\right)
$$

Since $B_{x 1}\left(E_{1}\right)=\overline{\eta_{x}\left(E_{1}\right)}$, comparing to Eq. (III-A.4), it is seen that $\bar{E}_{x}=E_{x}\left[E_{1}, \overline{\eta_{x}\left(E_{1}\right)}\right]$. The average energy of the other reaction product, $\bar{E}_{y}$, may either be obtained from $E q$. 


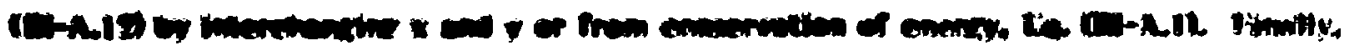

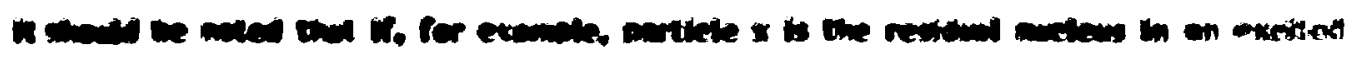

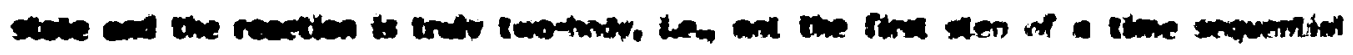

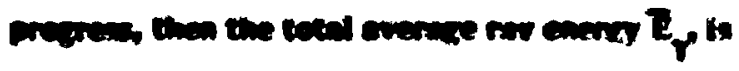

$$
\tau_{\mathbf{Y}}=\mathbf{w}_{\mathbf{x}}
$$

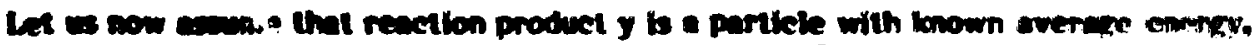

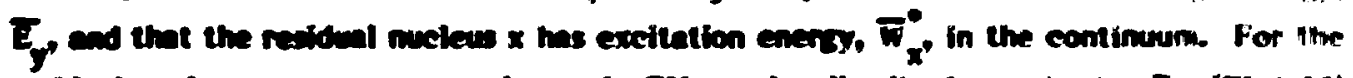

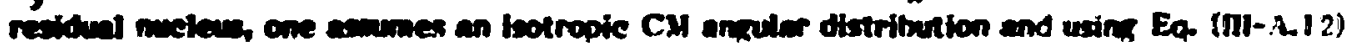
and solving for $Q$ (an average value since the transition is to the continum).

$$
\bar{Q}=\left(\frac{M_{1}+M_{2}}{M_{y}}, \bar{E}_{x}-\left[\frac{M_{1} M_{x}+M_{2} M_{y}}{M_{y}\left(M_{1}+M_{2}\right)}\right] E_{1}\right. \text {. }
$$

This equation has two unknowns, $\overline{\mathrm{Q}}$ and $\overline{\mathrm{E}}_{\mathrm{x}}$. For the other equation, one uses conservation of averuge energy from Eq. (III-A.I),

$$
E_{1}+\bar{Q}=\bar{E}_{x}+\bar{E}_{y}
$$

along with the definition of $\bar{Q}$ from Ea. (III-A.2),

$$
\overline{\mathbf{Q}}=Q_{0}+W_{2}^{*}-\bar{W}_{x}^{*} .
$$

Solving these relations

$$
\bar{w}_{x}^{*}=Q_{0}+w_{2}^{*}+\frac{\left(M_{1} M_{y}+M_{2} M_{x}\right)}{M_{x}\left(M_{1}+M_{2}\right)} E_{1}-\frac{\left(M_{1}+M_{2}\right)}{M_{x}} \bar{E}_{y},
$$

and

$$
E_{x}=\frac{M_{1}\left(M_{x}-M_{v}\right)}{M_{x}\left(M_{1}+M_{2}\right)} E_{1}+\frac{M_{y}}{M_{x}} \bar{E}_{y} .
$$

If this is the final end point of the reaction, i.e., no subsequent decay processes, then the total average gam ma ray energy is

$$
\bar{E}_{\gamma}=\bar{W}_{x}^{*}
$$

$$
\mathrm{III}-19 / 11 i-20
$$




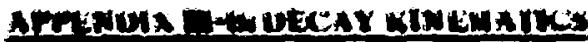

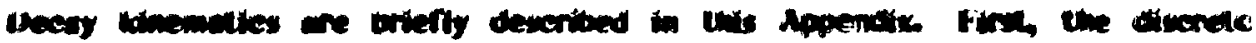

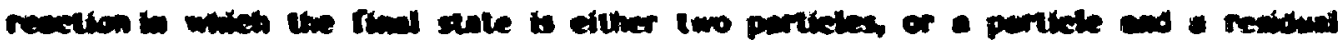

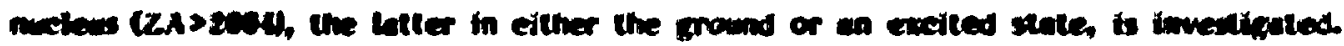

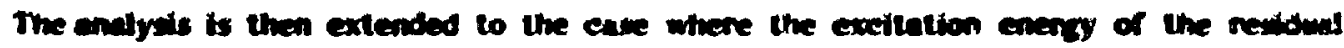

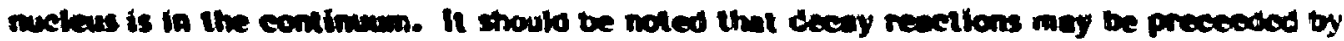

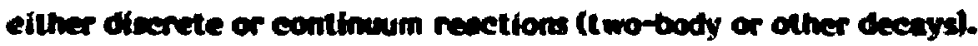

The diberete decay reuction is defined here as a secoil muless, energy $E_{1}$. decaying into iwo reaction products $x$ and $y$ with energies $E_{x}$ and $E_{y}$ respectively. Conservation of enersy in the laboratory (L) frame yields

$$
E_{1}+Q=E_{x}+E_{y}
$$

where the reaction $Q$ value is defined by Eqs. (III-A.2) and (111-A.3) of Appendix III-A with $W_{2}^{*}=M_{2}=0$. To obtain the pertinent energy of particle $x, E_{x}$, one transforms to the center of mass (CM) system, utilizes energy and momentum conservation, and transforms beck to the $L$ frame to obtain

$$
E_{x}=\frac{M_{x}}{M_{1}} E_{1}+\frac{M}{M_{1}} Q+\frac{2}{M_{1}}\left(M_{x} M E_{1} E_{1}\right)^{1 / 2} n_{x}
$$

where $z_{x}$ is the decay cosine of particle $x$ in the CM f rame. It has been assumed in Eq. (III-B.2) that $M_{1}=M_{x}+M_{y}$ which neglects $Q / c^{2}$, the nonrelativistic approximation.

The average final particle energy, $\bar{E}_{x}$, can be obtained analagous to the results of Eqs. (III-A.8) - (III-A.12) of Appendix III-A. Simply quoting the results, one obtains

$$
\bar{E}_{x}=\frac{M_{x}}{M_{1}} E_{1}+\frac{M_{y}}{M_{1}} Q+\frac{2}{M_{1}}\left(M_{x} M_{y} E_{1} Q\right)^{1 / 2} g_{x_{1}}\left(E_{1}\right),
$$

where $\mathrm{g}_{\mathrm{x}_{1}}\left(\mathrm{E}_{1}\right)$ is given by Eq. (III-A.11) of Appendix III-A. In general the angular distribution of the decay products is not known and the valid assumption is made that the decay is isotropic, i.e., $g_{x_{1}}\left(E_{1}\right)=0$. Equation (III-B.3) then reduces to

$$
\bar{E}_{x}=\frac{M_{1}}{M_{1}} E_{1}+\frac{M y}{M_{1}} Q \text {. }
$$




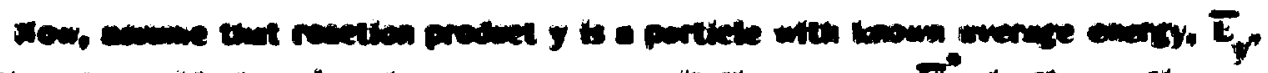

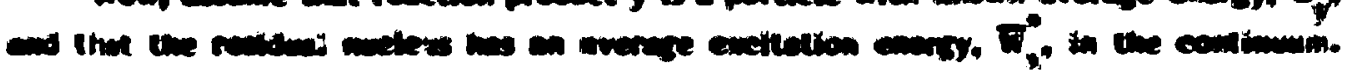
From En ortin

$$
E_{x}=\frac{u_{z}}{u_{1}} E_{1}+\frac{m_{y}}{m_{1}},
$$

wher. The $Q$ value is an averege since the trandition to to the contimoum. Analogous to

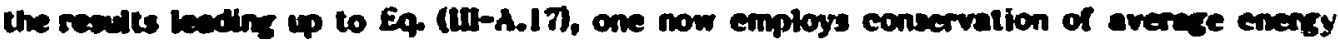
uns Eq (II-B.J) and the definition of the $Q$ "alue,

$$
\bar{Q}=Q_{0}+w_{1}^{*}-\bar{w}_{x}^{*}
$$

and solving these expressions

$$
\bar{w}_{x}^{*}=Q_{0}+w_{1}^{*}+\frac{M_{y}}{M_{x}} E_{1}-\frac{M_{1}}{M_{x}} \bar{E}_{y},
$$

and

$$
\bar{E}_{x}=\left(\frac{M_{x}-M_{y}}{M_{x}}\right) E_{1}+\frac{M_{y}}{M_{x}} \bar{E}_{y} .
$$

If this stage is the final stage of the reaction, then the total average gamma ray energy is

$$
\bar{E}_{Y}=\bar{W}_{X}^{*}
$$




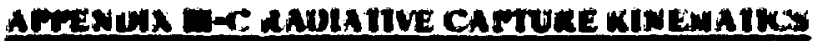

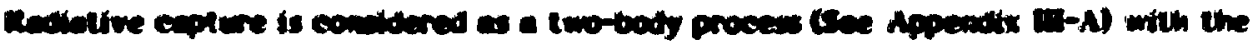

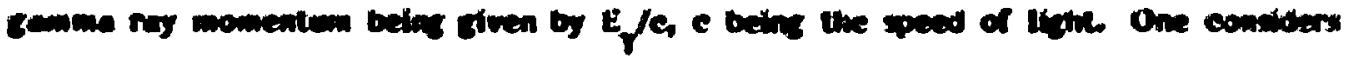

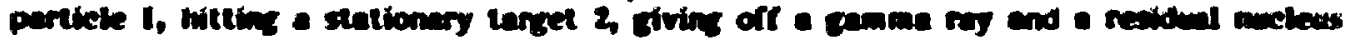
with enengies $E_{y}$ and $E_{x}$ respectively. Proceeding with enensy and unomenturn conbervation and aveuming center of mass (CM) botropy of the reaction products, as with the andycis leading to Eg (III-A.12) of Appendix III-A, gives

$$
E_{\gamma}=M_{x} e^{2}\left((1+z)^{1 / 2}-1\right),
$$

where

$$
\begin{aligned}
& z=\frac{2}{M_{x} e^{2}}\left(\frac{M_{2}}{M_{1}+M_{2}} E_{1}+Q\right), \\
& Q=Q_{0}+\left(w_{1}^{*}-w_{x}^{*}\right),
\end{aligned}
$$

and

$$
Q_{0}=\left(M_{1}+M_{2}-M_{X}\right) c^{2}
$$

Now, for $z<<1$, as is always the case,

$$
(1+z)^{1 / 2}=1+\frac{z}{2}
$$

Equation (III-C.1) then becomes

$$
E_{\gamma}=\frac{M_{2}}{V_{1}+M_{2}} \quad E_{1}+Q \text {, }
$$

and additionally,

$$
\bar{E}_{x}=\frac{M_{I}}{M_{1}+M_{2}} \quad E_{1} \text {. }
$$

In the above analysis, a single gamma ray had been assumed. If one assumes a cascade of gamma-rays and the same assumptions as used previously, then the same results hold and the total average gamma-ray energy is also given by Eq. (IIl.2). 


\section{arentinct:}

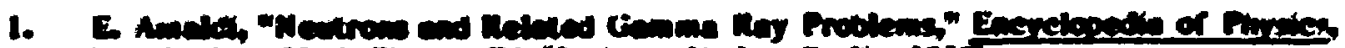

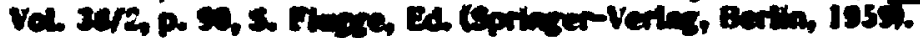

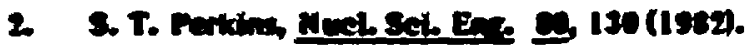


Chapter IV GAMCHK

IV - 1 


\section{GuHCrix}

\section{A Intmuviction}

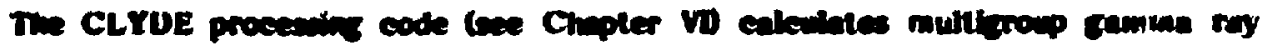

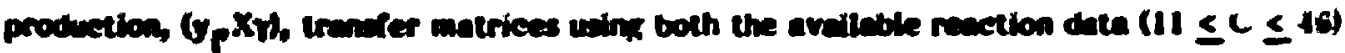

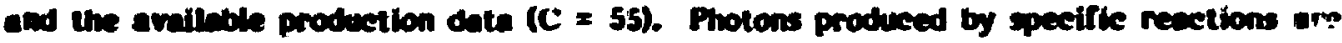
included wherever these data can be identified with the reaction. in most eases, however, and epecially at incident particle energies above the threahold of inelestic seatterin resctions, such identification cannot be made, so that photon production is lumped into a total photon prrduction cross section and associated speetra. The reaction information ineludes cross sestions $(I=0)$, energy spectra $\left(I=4, y_{0}=7\right.$, multiplicities $(I=$ $9, y_{0}=n$, and possibly angular distributions $\left(I=1, y_{0}=\eta\right)$. These latter data are treated as uncorrelated with the energy spectra. Reactions may be considered as either continum $(S=0)$ or discrete level excitation $(S=1)$. The production information $C=55$ contains the same types of data, excluding the multiplicity which is included within the production cross section. Production data are also considered as either continuum $(S=0)$, or diserete gamma production $(S=3)$.

As noted above, for most reactions there is no secondary photon $\left(y_{0}=7\right)$ evaluated information given in the file. Whenever this information is not included in the reaction data, it is ineluded in the production data. Thus, when CLYDE uses both components, it expects to obtain the whole. The one type of data common to both reaction and production is the total average energy into the $\gamma$ and $B^{-}$fields $\left(I=10, y_{0}=7\right.$ and 9$)$. This information is calculated by the ENDEP code (see Section III). Using these data, one can calculate the rate coefficients, $\overline{\mathrm{E}_{\gamma} \sigma_{R}}$, summed over all reactions for which there are no specific evaluated $\left(y_{i}, X \gamma\right)$ data given for $y_{0}=7$, and compare it to the equivalent sum over all $\gamma$ and $B^{-}$production components. This is what the GAMCHK code does. For energy conservation, the two sums should be equal, with any difference indicating how badly conservation is violated.

\section{B. TECHNIQUE}

Define $\bar{E}_{\gamma}(E)$ and $\vec{E}_{B}(E)$ as the $I=10, y_{0}=7$ and $I=10, y_{0}=9$ data respectively for $a$ given reaction. Furthermore, define $\sigma_{c}(E)$ as the corresponding reaction cross section. The same definitions apply to $\gamma$ ray production, $C=55$, and electron production, $\mathrm{C}=57$. A merged set of incident particle energies is obtained for all reaction and production data. All eross sections and $I=10$ data are obtained at this merged set of energies by linear interpolation. Note that $\overline{\mathrm{E}}(\mathrm{E}) \alpha(\mathrm{E})$ is no longer linear interpolable but is quadratically interpoleble. 


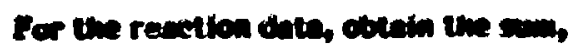

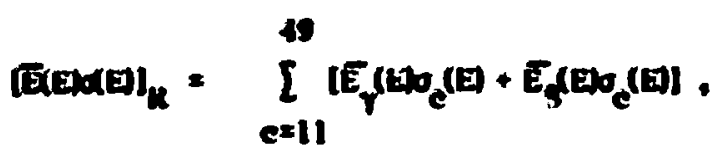

wheire reactions only eppear in the sum if there ere no evaluated camma or electron prodiluetion data given for the reaction at the incident energy in quetion.

For the produetion date, obtain the sum,

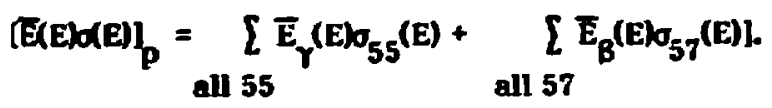

Then calculate the difference

$$
\Delta(E)=[E(E) d(E)]_{R}-[E(E) d(E)]_{p},
$$

and the $\%$ difference

$$
\% \Delta(E)=\frac{\Delta(E) \times 100}{[\bar{E}(E) \alpha(E)]_{R}}
$$

IN PUT, OPERATION, AND OUTPUT

The ingut file for GAMCHK contains the following information:

$\begin{array}{rll}\text { Columns } & \text { Format } & \text { Remarks } \\ :-3 & \text { F } 3.0 & \text { EPIA-A percentage error for printout. } \\ 4-15 & \text { E11.4 } & \text { BARDIN-An energy error for printout (MeV). } \\ 16-21 & \text { i6 } & \text { N NZAB-Beginning target ZA number. } \\ 22-27 & \text { i6 } & \text { NNZAE-Ending target ZA number. } \\ 28-30 & \text { i3 } & \begin{array}{l}\text { NPNTOP-If }>0, \text { increasing amounts of debug output are } \\ \text { given. }\end{array}\end{array}$

The calling sequence for $\mathrm{GAMCHK}$ is:

omega gamchk (input file) (box no.) (hsp option) (library name) 0 / TV 


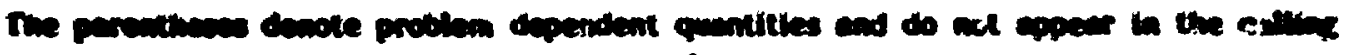

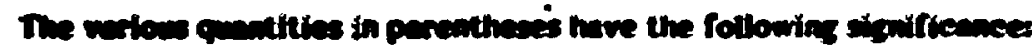

inpet rive

bour no.

hep eption

library name

0

\section{the flle requined by GAMCHK for inpent}

unats box number

GAHCHK produces output that is descriptive and/or dingostic for the probiem. This output may be direeted to the FRBu (use "freor for option), the high speed printer (use "nep" for option). or kept for inepection (use "deburo" for option) and/or outpur to RJET or other inedium using ALLOUT.

the library file of evaluated data used by GAMCHK.

The zero in this position indicates that the library exists as a file. The zero must be included in the calling sequence.

An example of running GAMCHK using an input file GAMIN with a library called ND821201 would be

omega gamchk gamin T61 debug nd821201 0/.9.999

The output consists of lines for each isotope containing:

1) incident particle energy

2) C $<55-\mathrm{Eg}$. (IV.I)

3) $\mathrm{C}=55,57-\mathrm{Eg}$. (IV.2)

4) difference - Eg. (IV.3)

5) $\%$ - Eq. (IV.4)

If the difference is negative, $\%$ will be followed by ***. If the inciaent energy is one of those in the production component $(C=55,57), \%$ is followed by ( $)$. It should be noted that only when Eç. (IV.3) > BARDIN and then also Eq. (IV.4) > EPIA is there printout.

$$
\text { Iv }-5 / 1 V-6
$$


chapten v UTLTY

I

$$
v-1 / v-2
$$




\section{Y. Hmurr}

\section{a. Intmourction}

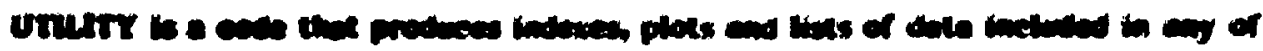

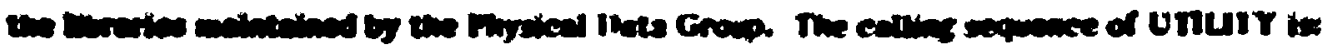

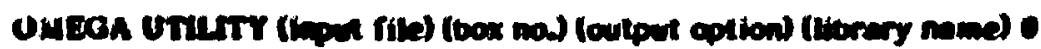

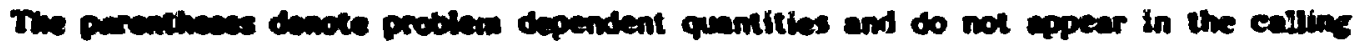
sequance. The vertom quatities in parentheses have the folbowing significances

input tile

box no.

output option

library name

D the file required by UTILTY for input

user's box number

UTUITY produces indexes, plots or lists of data as requested by the input file. This output may be direeted to the FR80 (use "fr80" for option), the high speed prir.ter iuse "hsp" for optionl, or kept for inspection (use "debug" for option) and/or output to RJET of other medium using ALiOUT.

the library file that is being used

The zero in this position indicates that the library exists as a file. The zero must be included in the calling sequence.

An example of running UTILITY using an input file INUT with a library called ND821201 is:

\section{B. PREPARATION OF IN PUT}

The format of the input file that must be used with UTILITY is:

$$
\text { i2, 2i6,2(1x,el1.4). }
$$

Any number of lists or indexes may be run at one time by including seperate requests in the input file. Problems that produce plots must be run seperately but any number of plat requests may be included in an input file for plot runs. The significance of the fields of the records in the input file is: 


\begin{tabular}{|c|c|c|}
\hline \multirow[t]{8}{*}{$1-2$} & $\mathbf{1}$ & an \\
\hline & 2 & 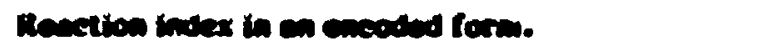 \\
\hline & 4 & Plets of the date with hardecps outphe \\
\hline & 24 & Prots of the dute with wieno-tiche ortpin. \\
\hline & 5 & $\begin{array}{l}\text { Labeled listings of all the dalie Total and } \\
\text { non-elatic eros sections are included where } \\
\text { eppropriate. }\end{array}$ \\
\hline & 25 & Same as 5 except that only cross sections are listed. \\
\hline & 6 & $\begin{array}{l}\text { Listing of cross sections and energy deposits for } \\
\text { each reaction. Note that these quantities are also } \\
\text { included with the rest of the data under a value of } \\
5 .\end{array}$ \\
\hline & 8 & 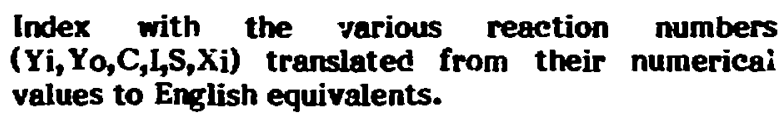 \\
\hline $3-8$ & ZA-Min & Minimum Value of $\mathrm{ZA}$. \\
\hline 9-14 & ZA-Max & Maximuin Value of ZA. \\
\hline $16-26$ & $\operatorname{Min}-E$ & $\begin{array}{l}\text { Minimum value of the level energy of a target } \\
\text { nucleus to be considered. }\end{array}$ \\
\hline 28-38 & $\operatorname{Max}-\mathrm{E}$ & $\begin{array}{l}\text { Maximum value of the level energy of a target } \\
\text { nucleus to be considered. }\end{array}$ \\
\hline
\end{tabular}


chapter vh clyut

$$
v I-1 / v 1-2
$$




\section{1. curue}

\section{A. Inthovection}

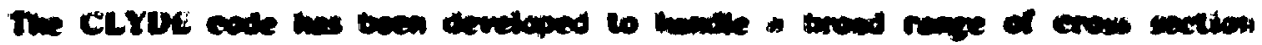

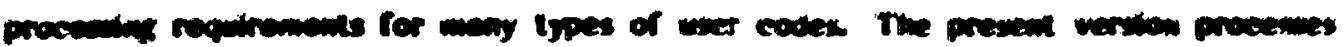

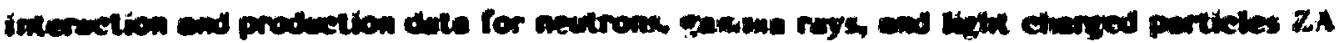

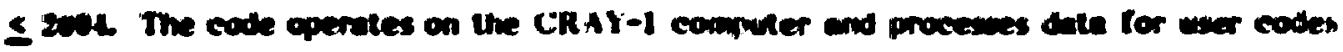

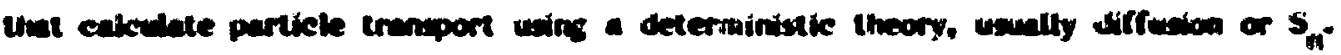

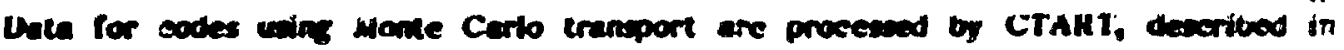
Chmpter vill.

The evaluated nuclear dala that are used by CLYUE must be in the form of a disk file in the binary mode of one of the Evaluated Nuclear Data Libraries (ENDL, ECPL, or EGUL). The user inust specify group boundaries, weighting fluxes, and the tanget isotopes for which processed data are reouired. Certain sets of group boundaries and weighting fluxes are standard and can be used by specifying thera in the ASCI file, BDFLS (see Appendix vil.B).

The code calculates flux-averaged group cross-sections, transfer matrices, total average energies associated with each outgoing particle field, isotope production multiplicities, and appropriate group averages of collateral quantities such as the average number of neutrons per fission.

CLYDE has the jollowing general characteristics:

1. For all practical purposes, it is unlimited in t're amount of data and the number of groups it can handle. It has a practical limit (approximately 10), to the order of transfer matrices tha: it should be used to generate.

2. The appropriate data for any material can be calculated for a standard group structure and scattering order in a reasonable amount of computer time.

3. It does not have any particular model or parameters built ir.

4. Redundant forms of input information that are allowed in the evaluated library are not processed by CLYLE.

5. Since both the input data and evaluated library are arranged in increasing energy, the code operates from low to high energy. The transport code calculations, on the other hand, usually compute from the greatest energy group to the least. Therefore, the code inverts all output arrays so that numbering and listing of groups and cross section begins with the greatest energy group. With each code run, a printout is produced that includes all pertinent information in a convenient form.

6. Units are cross sections in barns, energies in $\mathrm{MeV}$, and speeds in $\mathrm{cm} / \mathrm{shaike}$ ( 1 shake $=10^{-8}$ seconds).

$$
\text { VI }-3
$$




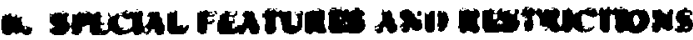

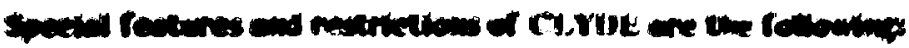

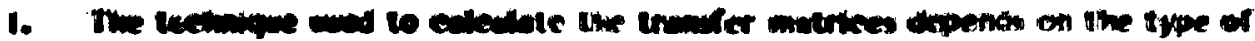

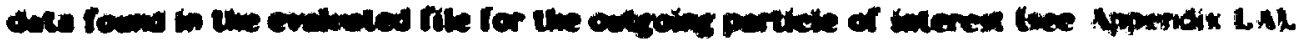

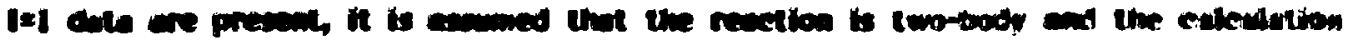

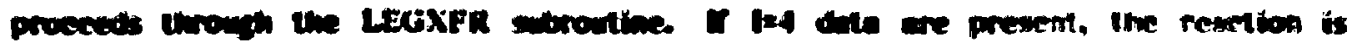

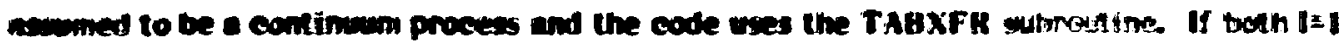

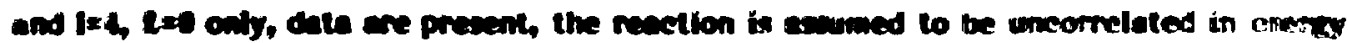
and engle and the angular distribution is asumed to be in the labaratory (I.) systeni. The Ist dula for $2>0$ are then caleulated from the relation'

$$
\pi_{2}(E+E)=\pi_{0}(E+E) f^{2}(E)
$$

Here, $f^{2}(E)$ is the $l^{\text {th }}$ Legendre coefficient of the $l=l$ date in the $L$ systern, e.g., $f^{\prime}(E)=$ TE), the average collision cosine.

2. All one dimensional arrays, e.g., $(E)$, are linearly interpolable in ineident energy. I=I data are linearly interpolable in cosine and also incident energy. I=4 data are linearly interpolable in final particle energy and also in sealed ineident energy after making the unit base transformation (see Sec. VI-C). I his latter case preserves linearity in interpolated areas.

3. The $\ell=0$ particle interaction inatrices for each reaction are renormalized

$$
\sigma_{o_{g+h}}=\sigma_{g+h}\left(\frac{m_{g} \sigma_{g}}{\sum \sigma_{h}}\right),
$$

where $m_{g}$ is the reaction multiplicity and $\sigma_{g}$ is the reaction cross section.

4. The $\ell^{\text {th }}$ moment of the angular flux expansion, $\varphi^{\ell}(E)$, is used to weight all $\ell^{\text {th }}$ order terms. If the input spectrum do not extend up to the order required, i.e., $\ell_{\max }+l$, CLYDE will use the highest order available to calculate the remaining terms up to the order required.

5. Evaluated data and weighting spectrum must span the group bounds of the incident particle.

6. The group bounds on the outgoing particle are arbitrary. Transfer below the lower bound of the lowest outgoing energy group is put into the lowest group. Transfer above the upper bound of the highest outgoing energy group is put into the highest group. Particles are therefore conserved. 


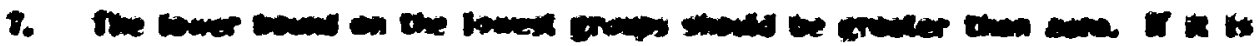

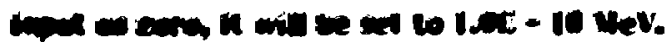

\section{c. clroe equatuas}

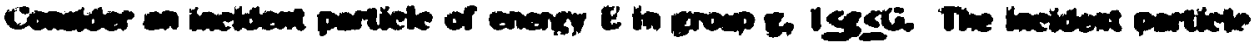

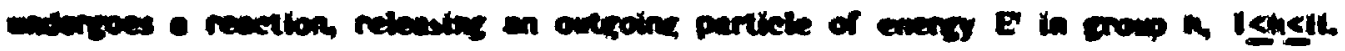

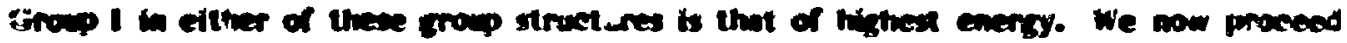
with the varions weltizrop relations ubed in CLYUE.

\section{Trenter Mntrix Dellaillon.}

CLYDE, upon input option can calculate several different types of transfer matrices. Consider the mumerator of the multigroup transfer matrix eleme.,t for a specific reaction (given $\mathrm{l}=1$ or $\mathrm{l}=4$ data)

$$
J_{g-h}=\int_{g} d E \nabla^{\ell}(E) m(E) \sigma(E) z_{h}^{l}(E) .
$$

The inner integral, $z_{h}^{l}(E)$, is written in a general form as

$$
z_{h}^{l}(E)=\int_{h} \pi_{\ell}\left(E+E^{\prime}\right)\left[(1-W)+w E^{\prime}\right] d E^{\prime}
$$

where $W$ is a selection parameter. Setting $W=0$ leads to a particle conserving matrices. Energy conserving matrices are obtained by setting $W=1$. T:ie input index, IECFLG, specifies the type of matrix calculated and its final deposition:

1. IECF LG=0. The selection parameter, $W$, is set equal to zero and the particle conserving matrices are calculated for all $\ell$,

$$
\sigma_{\ell_{g \rightarrow+}}=\frac{\int_{\mathrm{g}} \mathrm{dE} \varphi^{\ell}(\mathrm{E}) \alpha(\mathrm{E}) \int_{\mathrm{h}} \pi_{\left.\ell^{\left(E+E^{\prime}\right.}\right) \mathrm{dE}}}{\int_{\mathrm{g}}^{\mathrm{dE}} \varphi^{\ell}(\mathrm{E})} .
$$

2. $\operatorname{IECFLG}=1$. $W$ is set equal to one and the energy production conservation matrix, as has been used in gainma-ray production, is calculated for $\ell=0$, 


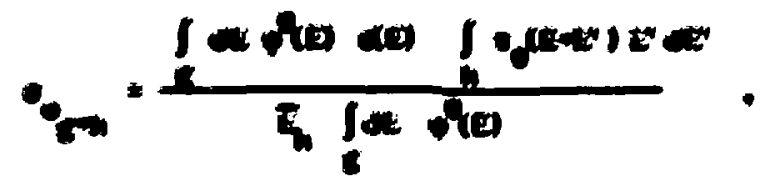

(11)

$$
E_{n}=\frac{1}{2}\left(E_{n+1}+E_{n}\right)
$$

(ทㄱ)

and is, eten are the grom bounderites.

3. HCFLGe2 beomes 1 and the eneny conserving matrix is cakeulated for $t=0$,

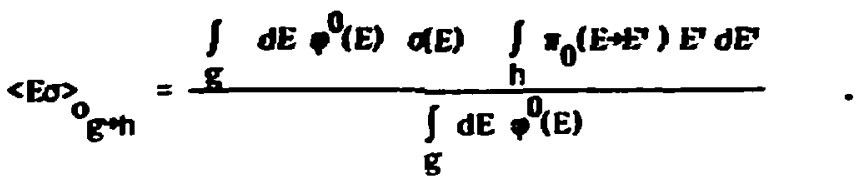

3. IECFLG=3. Group-split matrices, conserving both particles and energy, as used in charged particle production and transport. ${ }^{2}$ Initially, the particle conserving matrices, IECFLG $=0$, are calculated for all $\ell$. Then $w$ is set to one and the energy conserving matrix, IECFLG=2, is calculated for $\ell=0$. The average energy of the outgoing partieles in group $h$ (for a given ineident group $g$ ) is then

$$
\langle E\rangle_{h}=\frac{\langle E \sigma\rangle_{g-h}}{\sigma_{g+h}} .
$$

However, under group splitting, the energy of all particles in a group is the arithmetic average, $\overline{\mathrm{E}}_{\mathrm{h}}$, given by Eq. ( 1.7). Defining

$$
\tilde{\sigma}_{g+1}
$$

as being a matrix element after splitting, number and energy conservation for groups $h$ and $h+l$ yield

$$
\begin{aligned}
& \sigma_{o_{g}+h}=\tilde{\sigma}_{o_{g+h}}+\tilde{\sigma}_{o_{g+h+1}}, \\
& <E\rangle_{h} \sigma_{o_{g+h}}=\bar{E}_{h} \tilde{\sigma}_{o_{g+h}}+\bar{E}_{h+1} \tilde{\sigma}_{o_{g+h+1}} .
\end{aligned}
$$




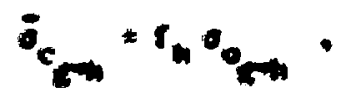

(utre)

$$
\sigma_{0_{n+1}}=\left(1-t_{n}\right) \sigma_{0 \times n} \cdot
$$

(vis)

where

$$
t_{h}=\frac{E_{h}-E_{h+1}}{E_{h}-E_{h+1}} .
$$

These relations are only valid for $\langle E\rangle_{h} \leq E_{h}$, since one must have $0 \leq f_{h} \leq 1$ for the elements after splitting to be non-negative. Similarly, for groups $h$ and $h-1$, one cbtains

$$
\begin{aligned}
& \bar{\sigma}_{o_{\text {Gh }}}=f_{h} \sigma_{o^{-h}} \\
& \tilde{\sigma}_{o_{g+h-1}}=\left(1-f_{h}\right) \sigma_{g-h}
\end{aligned}
$$

where here,

$$
f_{h}=\frac{\bar{E}_{h-1}-<E_{h}}{\bar{E}_{h-1}-\bar{E}_{h}},
$$

which is valid for $\langle E\rangle_{h} \geq \bar{E}_{h}$. After splitting for all $\mathrm{H}$ groups (for a given incident group g), group $h$ may have received components from itself and from the two adjacent groups. Thus, group splitting repartitions the transfer matrix in order to insure both energy and particle conservation. The average energy into the particle field in question after splitting is simply

$$
\langle E \tilde{\sigma}\rangle_{\mathrm{g}}=\sum_{\mathrm{h}}\langle\mathrm{E} \tilde{\sigma}\rangle_{\mathrm{g}+\mathrm{h}}=\sum_{\mathrm{h}} \overline{\mathrm{E}}_{\mathrm{h}} \tilde{\sigma}_{\mathrm{g}+\mathrm{h}} .
$$

There are, however, two cases in which splitting cannot be done, or one would be splitting out of the existing group structure. In these cases

$$
\tilde{\sigma}_{o_{g-h}}=\sigma_{o_{g+h}}
$$




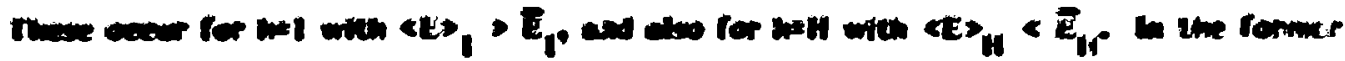

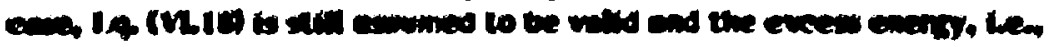

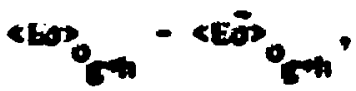

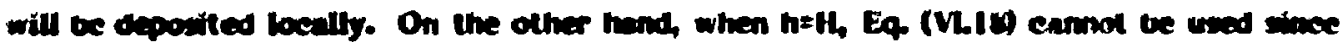
it would fimply 200 much energy imto the ourgoing partiele fleld and therefore too little local deposition One theretore uses

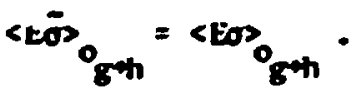

Including these two cases, the average energy into the particle field after splitting, to (V.18), is generalized to

$$
\begin{aligned}
& <E \tilde{\sigma}_{\mathbf{g}}=\sum_{h} \bar{E}_{h} \sigma_{\sigma_{G+h}} \quad \text { if }<E_{\rangle_{H}} \geq \bar{E}_{h} \text {, } \\
& =\sum_{h} \vec{E}_{h} \tilde{\sigma}_{\mathbf{o}_{g+h}}-\left[\overline { E } _ { \mathbf { H } } \tilde { \sigma } _ { \mathbf { o } _ { g + H } } - \langle E \sigma _ { \mathbf { o } _ { \mathrm { G } \rightarrow H } } ] \text { if } \left\langleE_{\rangle_{H}}<\overline{\mathrm{E}}_{\mathbf{h}}\right.\right. \text {. }
\end{aligned}
$$

\section{Transport Correction Terms.}

Iransport corrections are used to take into account higher Legendre moments of both the angular flux and the transfer matrices which are truncated during the actual calculation. ${ }^{3}$ They cause modifications to both the group total cross section as well as the ingroup transfer matrix elements. 'These corrections, as used in CLYDE, consist of two parts.

Transport theory codes typically admit only one total cross section to be entered. The first correction accounts for the inexactness of weighting the total cross sections with an $\ell=0$ flux spectrum by supplying all higher orders of weighting up to $L$, where $L$ is the order above which the transfer matrices are truncated. Consider the azimuthally symmetric transport equation for simplicity. The removal term on the left hand side of this equation is $\Sigma_{t}(E) f(E, \mu), \Sigma_{t}$ being the total cross section and $f$ being the angular flux. The removal term is moved to the right hand side of the equation and incorporated into the Legendre expansion of the collision integral. The expression is then integrated over energy to obtain the multigroup form. Now define

$$
\text { VI }-8
$$




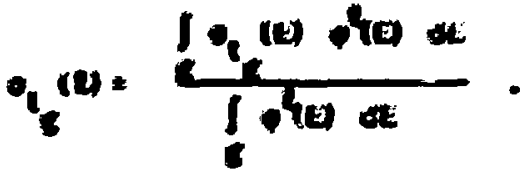

cine now ads

4. (1)

to both sides of the transport equation. The mieroscople total eroses section then is

$$
\sigma_{B^{\prime}}=\sigma_{t^{\prime}}(0)
$$

and the corrected transfer matrices, $0 \leq l \leq L$, are

$$
\sigma_{\ell_{g}+g^{\prime}}(\text { corr })=\sigma_{\ell^{\prime} \cdot g^{\prime}}+\left[\sigma_{t_{g}}(0)-\sigma_{t_{g}}(l)\right] \delta_{g} \cdot
$$

The second (and more familiar) transport correcting term arises from the truncation of the transfer matrices at some order $L$. This leads to the so called extended transport approximation. Consider again the azimuthally symmetric multigroup transport equation and subtract

$$
\Sigma_{L+1} f_{g}(\mu)
$$

from both sides of this expression where this cross section term is yet undefined. On the right hand side, incorporate this term into the Legendre moment expansion of the collision integral and break this resulting sum into the ranges of $0 \leq \ell<L$ and $L+1 \leq \ell \leq \infty$. In this latter sum, all $\ell^{\text {th }}$ moment transfer rates are assumed to be the same as for $\ell=L+1$, and requiring that this summation identically vanish, yields for the microscopic $L+1$ transport correcting cross section,

$$
\sigma_{\operatorname{tr} \text { corr }_{g}}=\sigma_{L+1}=\frac{\sum_{g^{\prime}} \sigma_{L+1}{ }_{g^{\prime}+g} \varphi_{g^{\prime}}^{L+1}}{\varphi_{g}^{L+1}} .
$$

The group cross section, $\sigma_{\mathrm{g}}$, then becomes

$$
\sigma_{g}=\sigma_{t_{g}}-\sigma_{\text {tr corr }}
$$

and the transfer matrix elements, $0 \leq l \leq L$, are 


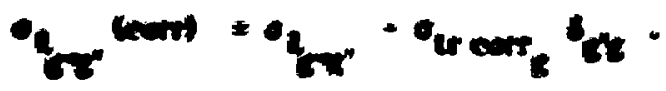

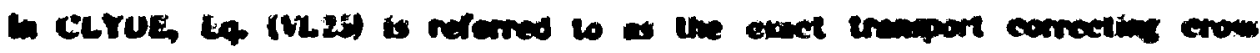

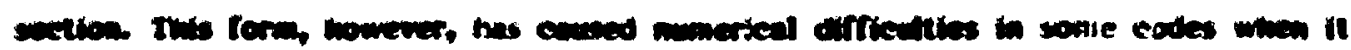

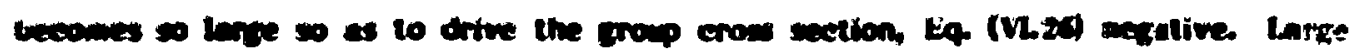

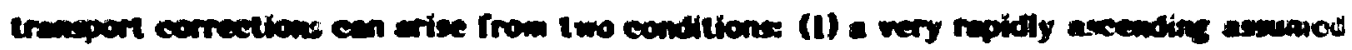
flut wetghting spectrum (which mby be inappropriale to the material on questionti (j) too low an order of Legendre expansion (In many cases this will be a code limitation) in the ease of a highly antsolropic scatterer. A negative diegonal transfer term can arise even in the abence of these conditions, If the group $q$ is suffietently small that the self-ceatterer term is less than the in-scatterers.

To help alleviate this problem, it is assumed in Eq. (VL-25) that the rate of transfer into group $g$ for order $L+1$ is equal to the rate of transfer out, which yields in this case

$$
\sigma_{\text {tr corr }}=\sum_{\mathbf{g}^{\prime}} \sigma_{L+1}
$$

This expression is referred to in the code as the approximate transport correcting cross section and is the more commonly used version, since it assumes no knowledge of the $L+I$ group fluxes. Excluding the conditions of high particle nultiplicity and large forward scattering, the approximate form cannot lead to a negative group cross section, although it can lead to a negative ingroup transfer matrix eleinent.

CLYDE has the additional option of lettirg the code choose the appropriate form for the transport correcting term. In this case, one starts with Eq. (VI.25) and if for any group Eg. (VL.26) becoines negative, then for that group Eq. (VI.28) is employed for the transport correcting cross section. One can also chnose to omit the transport correction altogether.

\section{Average Group Flux and Inverse Speed}

The group flux is calculated from

$$
\phi_{g}^{\ell} \equiv \int_{E_{g-1}}^{E_{g}} \mathrm{dE} \phi^{\ell}(E)
$$

for each order $\ell$ from 0 to $L+1$. Here, $\varphi^{\ell}(E)$ is the weighting spectrum used in generating the calculational constants from the evaluated data. 


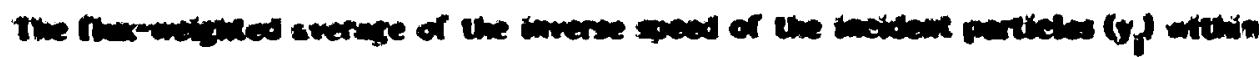

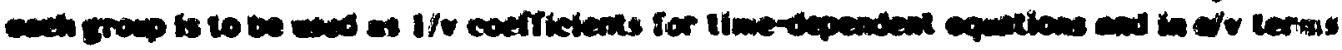

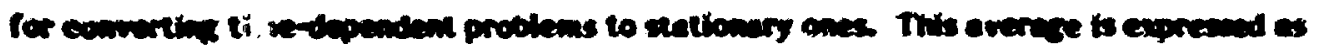

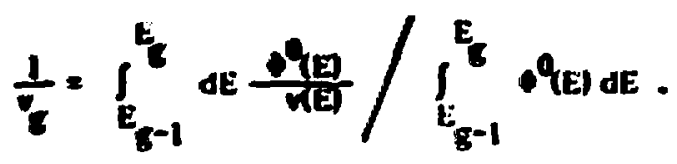

The analyic soltution to this equation for linearly interpolable ${ }^{0}$ in wegment i is

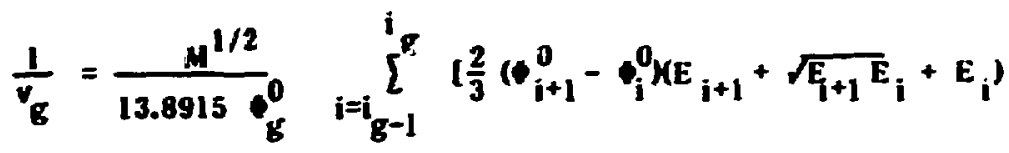

$$
\begin{aligned}
& \left.+\frac{2 E_{i+1}-E_{i}^{0}\left(_{i+1}^{0}\right)}{\sqrt{E_{i+1}}+\sqrt{E_{i}}}\right]
\end{aligned}
$$

Here the i's denote elements in the merged table of group bounds and flux energies from the input flux table. The numerical factor converts the speed to the units of $\mathrm{cm} / \mathrm{shake}$, where 1 shake $=10^{-8}$ sec; also $M$ is the atomic mass (amu) of the incident particle.

\section{Reaction Group Average Cross Sections}

The group averages of the linearly interpolable integrated cross sections are weighted with appropriate fluxes over the group structure. The averages are expressed by

$$
\sigma_{x_{g}}^{\ell}=\int_{E_{g-1}}^{E_{g}} d E \sigma_{x}(E) \Phi^{\ell}(E) / \int_{E_{g-1}}^{E_{g}} d E \Phi^{\ell}(E) .
$$

These quantities are generated for each $\ell, 0 \leq \ell \geq L+1$. The values obtained for $\ell>0$ are used in making the Legendre flux expansion correction to the total cross section, as discussed in the section on the transport correction.

The above integrals, being comprised only of products of linear functions, are analytic [see UNINT, DIINT, TRINT subroutine descriptions in Appendix (VI.A) ]. 


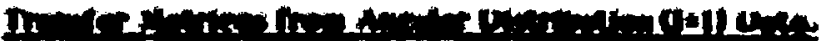

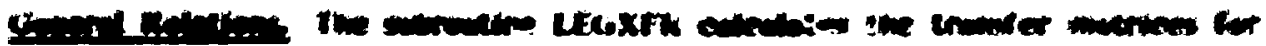

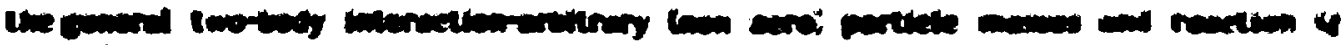

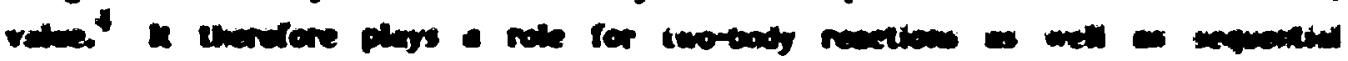

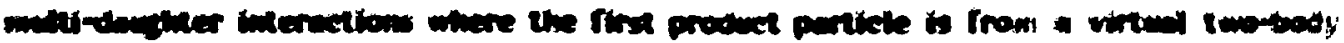

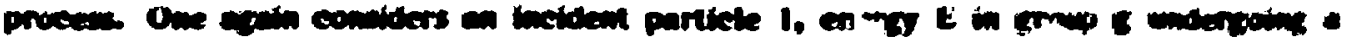

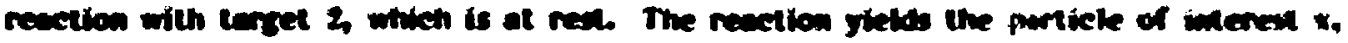

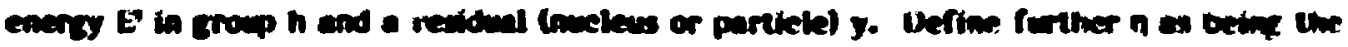
center of mus (Cu) eolltsion cuaine of partiele $x$ and $u$ as being it: leboratory (L) syatem

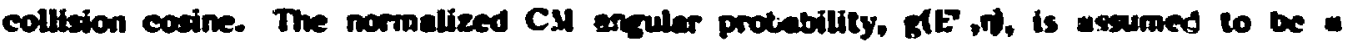
piecewise linear function. Under these conditions, the inner integral for the trensfer matrix can be evaluated analytically. 5

The integrals of interest for the transfer inatrix has been expreseed by Eq. (VL.3) and (VL4). For the general two-body reaction, the Legendre moments of the transference probability are given by

$$
z_{\ell}\left(E+E^{\prime}\right)=\left[\frac{d}{d E^{P}} n\left(E, E^{\prime}\right)\right] P_{\ell}\left[\mu\left(E, E^{\prime}\right)\right]_{g}\left(E, \eta\left(E, E^{\prime}\right)\right],
$$

where $P_{\ell}(\boldsymbol{W})$ is the Legendre coefficient of order $\ell$.

The kinematic relations between the incident particle energy $E$, the $L$ an CMI collision cosines of particle $x$, and its final energy $E$ ' are given in Refs. 4 and 5 . One has

$$
n=\alpha[\mu \pm r(\mu)]^{2}-\gamma
$$

and

$$
E^{\prime}=a_{l x} E[\mu \pm r(\mu)]^{2},
$$

$$
\text { where } \begin{aligned}
\alpha & =\frac{a_{1 x} E}{2\left[a_{1 x} a_{2 y} E\left(E-E_{1 \alpha}\right)\right]^{1 / 2}}, \\
r(\mu) & =\left(B^{2}+\mu^{2}\right)^{1 / 2}, \\
B^{2} & =\frac{a_{2 y}\left(E-E_{1 \alpha}\right)}{a_{I x} E}-1,
\end{aligned}
$$




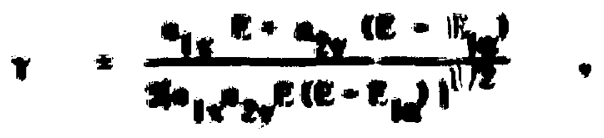

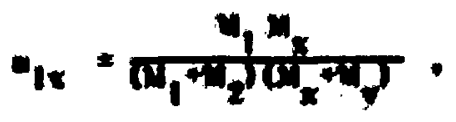

$$
\begin{aligned}
& -2 y=\frac{w_{2} m_{y}}{\left(W_{1}+w_{2}\right)\left(m_{x}+m_{y}\right.} \text {. }
\end{aligned}
$$

insul

(vinen

and

$$
E_{1 \alpha}=-\left(\frac{M_{1}+M_{2}}{M_{2}}\right) Q \text {. }
$$

$Y$ denotes particle mass, and $Q$ is the usual reaction $Q$ value. In Eq (VL.31) and (VL.35) and what follows, the $(+)$ sign is used, unless $\left(B^{2}\right)$ is less than zero. In that case the minimum $\mathbf{L}$ collision cosine is

$$
\mu_{\min }=\left(-\beta^{2}\right)^{1 / 2}=\left[1-\frac{a_{2 y}\left(E-E_{1 \alpha}\right)}{a_{1 x} E}\right]^{1 / 2},
$$

and the corresponding $\mathrm{CM}$ collision cosine is

$$
n\left(\mu_{\min }\right)=-\left[\frac{a_{2 y}\left(E_{1}-E_{1 a}\right)}{a_{1 x} E^{1 / 2}}\right]^{1 / 2} \text {. }
$$

This implies that the (+) sign in Eqs. (V1.34) and (VI.35) is to be used down to the energy

$$
E\left(\mu_{\min }\right)=a_{1 x} E-a_{2 y}\left(E-E_{1 \alpha}\right),
$$

and the $(-)$ sign then used at all lower energies.

Finally, from Eg. (VI.34),

$$
\frac{\mathrm{dn}}{\mathrm{d} \mu}= \pm \frac{2 \alpha}{\mathrm{r}(\mu)}[\mu \pm \mathrm{r}(\mu)]^{2}
$$

with the sign convention as described above. A special case arises when considering the recoil nucleus of elastic scattering, or when the emergent particles from elastic scattering are identical. In that case, [referring to Eqs. (IV.36) ], $a_{1 x}=a_{2 y}$ and $E_{1 \alpha}=0$, and 


$$
\begin{aligned}
& a n-1 \\
& = \\
& t=\log _{1 x^{2}} \\
& \text { min }=0
\end{aligned}
$$

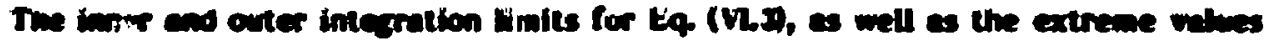
of the vartow parancters are stown in Fiz. 1. More detail ts civen in Ref. \&.

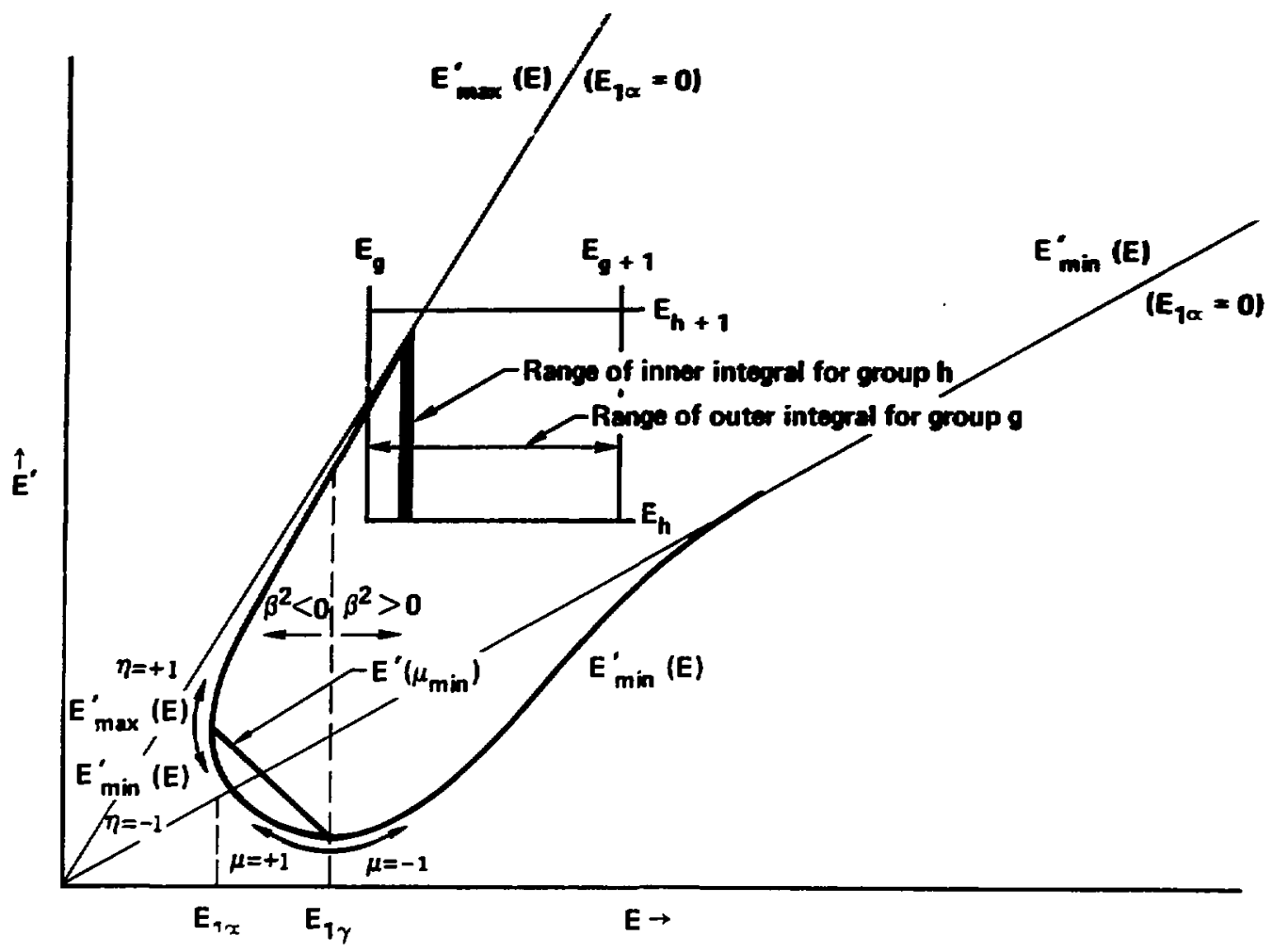

FIG. 1. Collision domain in the $\mathrm{E}^{\prime}, \mathrm{E}$ plane.

$$
\mathrm{VI}-14
$$




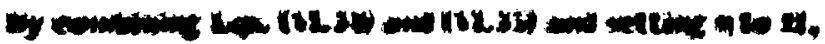

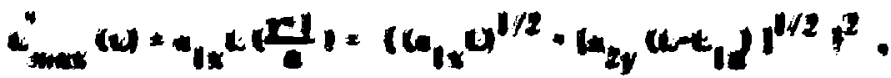

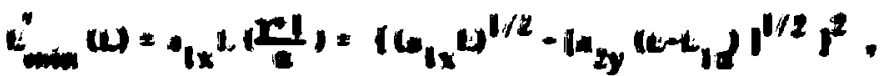

(Neres)

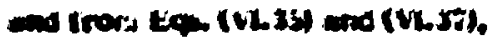

$$
\left.E^{\prime} u_{\text {was }}=a_{1 x}+u_{\text {min }}^{2}=a_{2 y} L_{1 a}-\omega_{2 y}-a_{1 x}\right)^{k}
$$

The lnoer Integral. The imner integral is expresced by to (VLt) in eonjunction with tq (VL33). Une now changes the integration variatile fron final particle energy, $t$ ', to its 1 gystein collision cosine, $h$

$$
L_{h}^{2}(t)=\int P_{l}\left(\mu g(E, \mu) \frac{d \eta}{d \mu}[(1-w)+w E(1)] d \mu\right.
$$

The CM angular probability datu are given in the form for a given $E_{j}$,

$$
-1, g\left(t_{i},-1\right), \ldots, \eta_{i j} g\left(E_{i}, \eta_{j}\right), \ldots, 1, g\left(t_{i}, 1\right)
$$

which are linearly interpolable both in incident erergy and in $\mathrm{CM}$ cosine. The functional form of the $g(E, n)$ data is $g=a_{j}(E)+b_{j}(E) n$. The $g(E, n)$ data are found by interpolating in (E) the linear data in $\eta$ at the surrounding data sets $a t\left(E_{i}\right)$ and $\left(E_{i+l}\right)$. The sets of breakpoints in $n$ at $E_{i}$ and $E_{i+l}$ may not be identical, so a new set of $n$ breakpoints at $E$ is constructed by inelding those for $\dot{k}_{i}$ and $E_{j+1}$. By inverting Eq. (VI.34) we calculate a corresponding set of breakpoints in ( $\omega$ ). In addition, a breakpoint at $\mu_{\min }$ is included, if appropriate. rinally, by inverting Lq. (VI.35), we include two breakpoints corresponding to $\left(E_{h}^{\prime}\right)$ and $\left(E_{n+1}^{\prime}\right)$, and two for $E_{\min }^{\prime}(E)$ and $\dot{E}_{\max }^{\prime}(E)$, providing they fall wit in the range $(-1<n<$ $+1)$ and $(-1<\mu<+1)$.

Equation (IV.48) is expressed now as

$$
z_{h}^{\ell}(E)=\sum_{k=k_{\text {nin }}}^{k(\mu} z_{-k}^{\ell}(E)+\sum_{k=k\left(\mu \min ^{\prime}\right)}^{k} z_{+k}^{\ell}(E),
$$




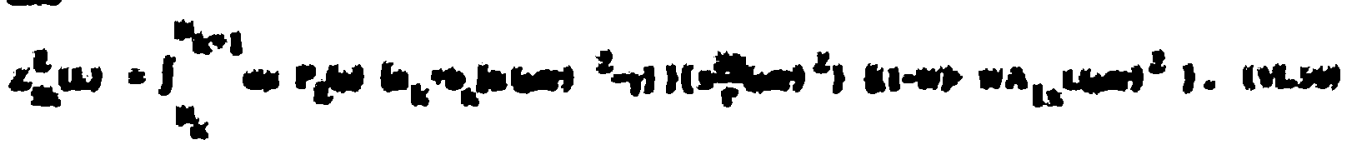

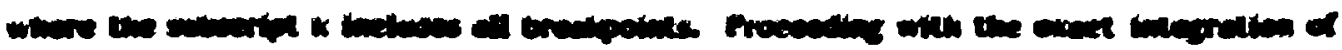
to Intill we rewrite the

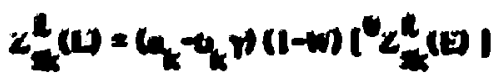

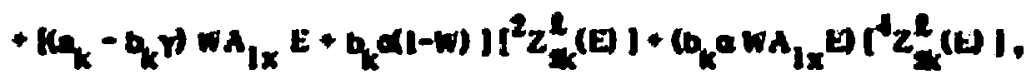

(vesi)

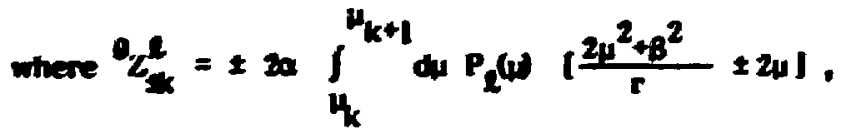

$$
2 \cdot Z_{ \pm k}^{2}= \pm 2 \alpha \int_{u_{k}}^{\mu_{k+1}} \omega P_{\ell}(\psi)\left[\frac{8 \mu^{4}+8 \beta^{2} \mu^{2}+\beta^{4}}{r} \pm\left(8 \mu^{3}+4 \beta^{2} w\right),\right.
$$

and ${ }^{4} Z_{t k}^{l}= \pm 2 \alpha \int_{\mu_{k}}^{\mu_{k+1}} d \mu P_{\ell}(\mu)\left[\frac{32 \mu^{6}+48 \beta^{2} \mu^{4}+18 \beta^{4} \mu^{2}+\beta^{6}}{r} \pm\left(32 \mu^{5}+32 \beta^{2} \mu^{3}+6 \beta^{4} \omega\right)\right]$,

where $r=\left(\mu^{2}+\beta^{2}\right)^{1 / 2}$.

Further, $P_{\ell}(\mu)$ may be expressed as a finite series

$$
P_{\ell}(\omega)=\sum_{m=\ell,-2}^{\ell} \frac{C_{m \ell}}{d_{\ell}} \mu^{n_{3}} \text {. }
$$

Equations (VI.52) then become:

$$
\begin{aligned}
& 0_{\mathrm{Z}_{ \pm k}^{\ell}}^{\ell}= \pm 2 \alpha \sum_{\mathrm{m}=\ell,-2}^{0} \frac{\mathrm{C}_{\mathrm{m} \ell}}{\mathrm{d}_{\ell}}\left(2 \Delta H_{\mathrm{m}+2}+\beta^{2} \Delta \mathrm{H}_{\mathrm{m}} \pm 2 \Delta \mathrm{F}_{\mathrm{m}+\mathrm{l}}\right),
\end{aligned}
$$

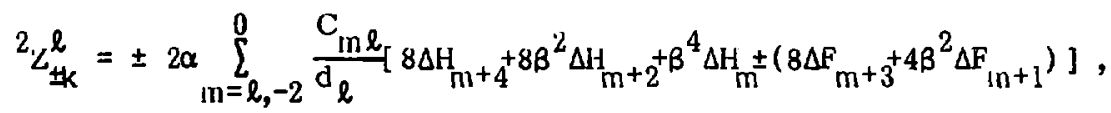




$$
\begin{aligned}
& 4 \cdot-1 \text {. }
\end{aligned}
$$

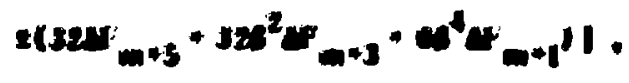

IrLsed

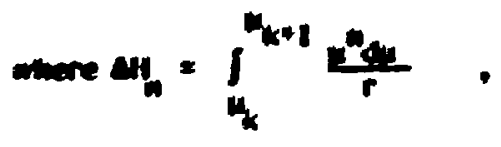

(VList)

and $\quad a_{n}=H_{n}\left(w_{k+1}\right)-t_{n}\left(w_{k}\right)=\frac{w_{k+1}^{n+1}}{n+1}-\frac{\mu_{k}^{n+1}}{n+1}$.

(VL55d)

The integration of (VL55a) proceeds as follows: The function $G_{n}=f_{1}^{n}$ rdu is integrated in two ways tirst by making use of the functional form of $r$ :

$$
\Delta \Delta_{n}=\int \frac{\mu^{n}}{r}\left(\mu^{2}+\beta^{2}\right) d \mu=\Delta u_{u+2}^{i}+\beta^{2} \Delta H_{n},
$$

and secondly by integrating by parts:

$$
\begin{aligned}
\Delta u_{n} & =\Delta\left(\mu^{n+1} r\right)-\int\left(n \mu^{n} r+\frac{\mu^{n+2}}{r}\right) d \mu, \\
& =\Delta\left(\mu^{n+1} r\right)-n\left(\Delta H_{n+2}+B^{2} \Delta H_{n}\right)-\Delta(1)_{n+2} .
\end{aligned}
$$

Equating the two forms of the integral brings us to an iterative expression for $\Delta H_{n+2}$ :

$$
\Delta H_{n+2}=\frac{n+1}{n+2}\left(\Delta\left(F_{n} r\right)-\beta^{2} \Delta H_{n}\right)
$$

The two base integrals, $\Delta \mathrm{H}_{0}$ and $\Delta \mathrm{H}_{1}$ are, from: siandard tables,

$$
\Delta H_{0}=\ln \frac{\mu_{k+1}+r_{k+1}}{\mu_{k}+r_{k}},
$$

and $\Delta H_{1}=r_{k+1}-r_{k}$.

In the special case of elastic scattering $\left(\beta^{2}=0\right)$, it is clear from inspecting Eq. (VI.55) that

$$
\Delta H_{n}=\Delta F_{n-1}
$$




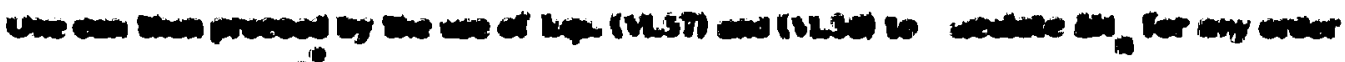

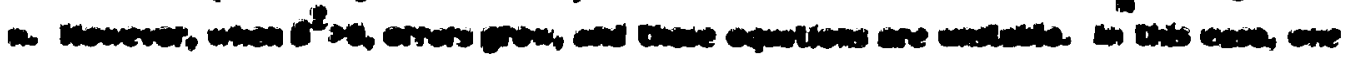

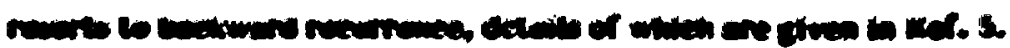

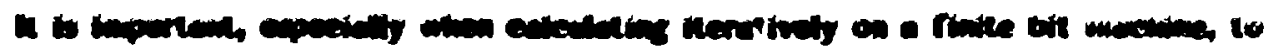

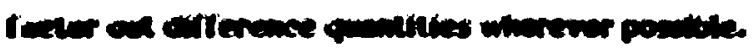

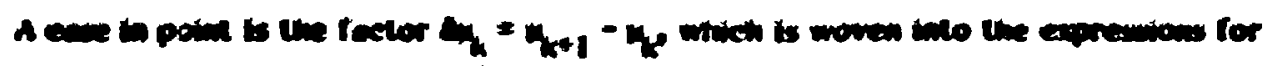

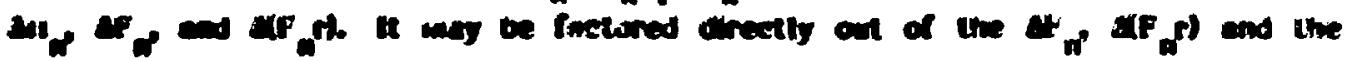

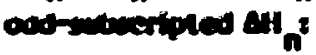

$$
\begin{aligned}
& \left.\Delta F_{n}=\frac{a}{(n+1)} u_{k+1}^{n}+u_{k+1}^{n-1} u_{k}+\ldots+u_{k+1} u^{n-1}+u_{k}^{n}\right) \\
& \Delta u_{1}=\Delta w_{k+1} \frac{u_{k+1}+u_{k}}{r_{k+1}+r_{k}}, \text { ele. }, \\
& \Delta\left(F_{n} r\right)=\frac{\left(\lambda_{k+1}^{n+2}+\mu_{k}^{n+2} \Delta F_{n+2}+B^{2}\left(u_{k+1}^{n+1}+\mu_{k}^{n+1}\right) \Delta F_{n+1}\right.}{\left(r_{k+1}^{n+2} r_{k+1}+F_{k}^{n+2} r_{k}\right)}
\end{aligned}
$$

The even ordered $\Delta H_{n}$ are not so easily factored; however, $\Delta H_{0}$ may be calculated by referring to its expression using center of mass cosines:

$$
\Delta H_{0}=\ln \frac{\mu_{k+1}+r_{k+1}}{\mu_{k}+r_{k}}= \pm \frac{1}{2} \ln \frac{\gamma+n_{k+1}}{\gamma+r_{k}},
$$

where the sign is plus if $\eta_{k}$ and $\eta_{k+1}$ are both greater than or less than $\eta_{1}\left(\mu_{\text {min }}\right)$. Where

$$
\Delta \pi \ll(\gamma+\bar{\pi},
$$

the first few terms of the logarithrnic expansion may be used.

The Outer Integral. The outer integral is given by Eq. (Vl.3). The evaluations of the outer integrals are done simultaneously for all $\ell$. These integrals are subdivided; the intervals being the same for all $\ell$. The bounds are determined at these various energies:

1. all the breakpoints for any $\ell$ in the flux $\left[\phi^{\ell}[l]\right.$ data;

2. the breakpoints in the cross-section $\left.\left[\alpha E_{i}\right)\right]$ data;

3. the breakpoints in the angular distribution $\left[g\left(E_{i}, n\right)\right]$ data;

4. the singular points at $E_{1 \alpha}$ and $E_{1 \gamma}$; 


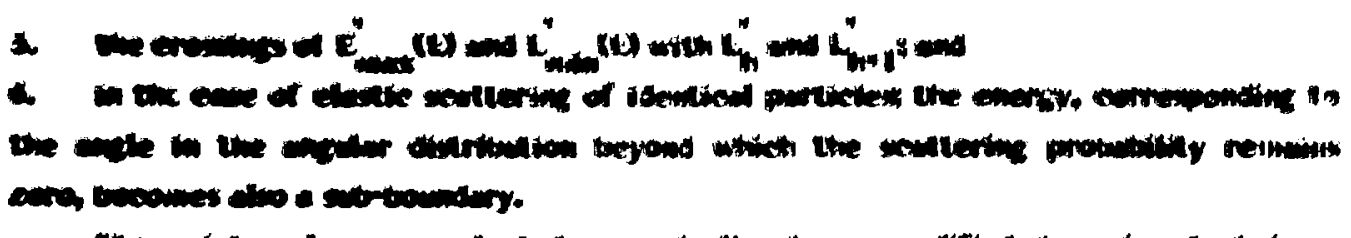

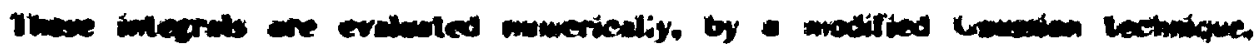

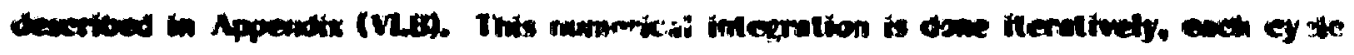

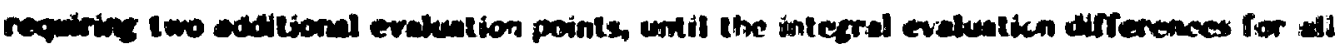

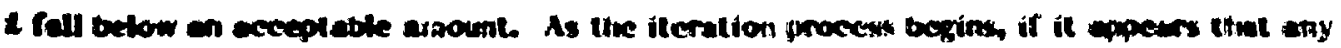
of the (unctions (ll) deing integroled are highly peaked in bi wt one soundury or the other. the peeked end is broken off and treated as another subintervul.

The final operation in computing the transfer inutrix clements is to divide the outer integral oy the group Rux, Eg. (VLi2y).

\section{Transer Probubility from Tabular Energy Listribution (l=t) Lata*}

Basic Uata. The tabular energy distribution data $(I=4)$ consist of a series ot incident energies $k_{i}$, at each of which is given a set of ellission energies $E_{i j}^{\prime}$ and their corresponding probabilities $\bar{\pi}_{i j}^{l}\left(E_{j}+E_{j j}^{\prime}\right)$. These points are assumed to be linearly interpolable in emission energy. Interpolation in incident energy, however, is not straightforward. There is a set of these data for each $\ell$ th Legendre coefficient of the expansion of the correlated energy-angle distribution data. The integrals to be evaluated are again given by Eqs. (VL.3) and (VI.4), the analysis being done in the TABXFR subroutine.

Unit Base Transformation. Simple linear interpolation in incident energy to obtain a distribution at an intermediate energy, $E$, would, in general, lead to an intermediate distribution that would have finite probabilities of transfer to energies above $E$, which in turn would lead to transfer probabilities that do not conserve energy. A scheme that avoids this would be to use linear interpolation in incident energy, $E$, of the end points, and to connect the break points of the line segment distributions based on their relative proportion of the energy span of the distribution, with a resulting distribution constructed by linearly connecting (in E) the interpolated (in $E^{\prime}$ ) probabilities. T'his process, however, does not result in the integral of the distribution at an intermediate energy being linearly interpolable, as would be desirable. If linear interpolation is used to connect two right trapezoids on parallel planes, the interinediate areas so constructed are not linear in the

* $I=3$ data are transformed to $I=4$ data for processing by CLYUE. 


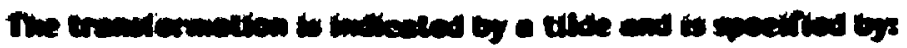

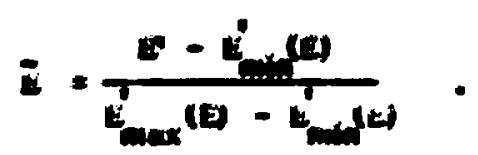

IVust

Comervation of probulitty under the teandoranation bacts toe

$$
\theta^{2}(t+\bar{E})=q^{2}(t+E)\left(E_{\max }^{\prime}(E)-\dot{E}_{\min }^{\prime}(E)\right) \text {. }
$$

The data are available in the form

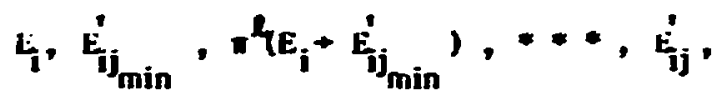

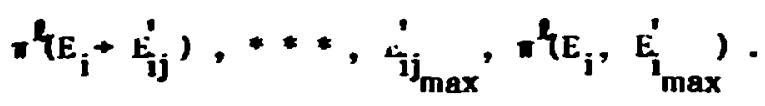

At $E_{j}$ Eqs. (VL.61) and (VL.62) are

$$
\tilde{E}_{i j}=\frac{\dot{E}_{i j}-\dot{E}_{i j}^{\prime}}{\dot{E}_{i j} j_{\max }-\dot{E}_{i j_{\min }}^{\prime}},
$$

and

$$
\pi^{l}\left(\dot{i}_{i}+\tilde{E}_{i j}\right)=\pi^{l}\left(E_{i}+E_{i j}^{\prime}\right)\left(E_{i j}^{\prime}-E_{\text {max }}^{\prime}\right),
$$

respectively. At all other energies (E) the distributions in $\widetilde{E}$ are determined by the following procedure:

1. The two $E_{i}$ table entries which span the desired incident energy $E$ are determined $\left(E_{i}, E_{i+1}\right)$, and their corresponding $E^{\prime}, \pi^{l}$ tables are transformed according to Eqs. (VI.63) and (Vl.64).

2. 'The break points and their corresponding $\pi^{\ell}$ 's on each of the transformed distributions are listed.

3. The two lists of break points are merged into an $\tilde{E}_{k}$ list. 


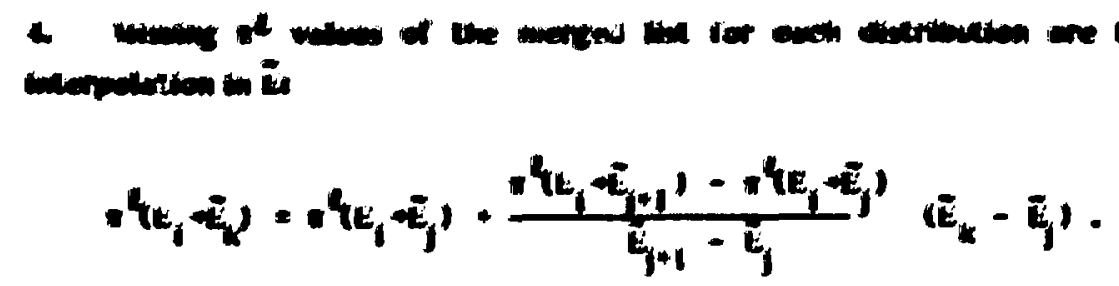

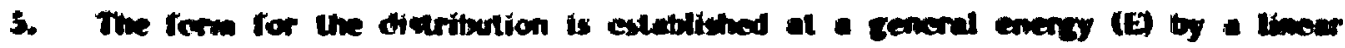
interpolation in enerzy between the foregoing quantitien:

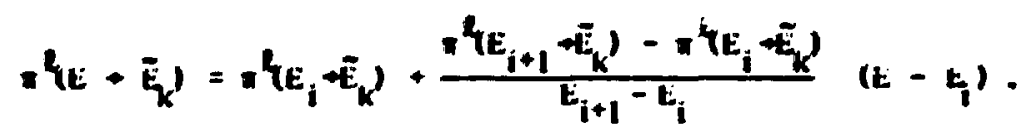

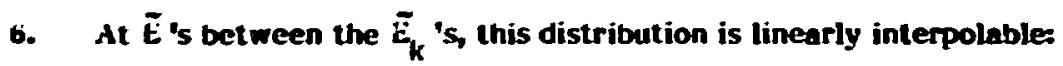

$$
\pi(E+\tilde{E})=\pi\left(E+\tilde{E}_{k}\right)+\frac{\pi^{2}\left(E+\tilde{E}_{k+1}\right)-\pi^{2}\left(E+\tilde{E}_{k}\right)}{\tilde{E}_{k+1}-\tilde{E}_{k}}\left(E-\tilde{E}_{k}\right)
$$

this is the general form needed for the inner integral, Eq. (VI.4). An example of the unit base transformation is shown in Fig. 2.

The Inner Integral. The inner integral, Eq. (VI.4), is restated after transforming to unit base coordinates:

$$
Z_{1}^{\ell}(E)=\int_{\tilde{E}_{h}}^{\tilde{E}_{h+1}} d \tilde{E} \pi^{\ell}(E+\tilde{E})\left[(1-W)+W E^{\prime}\right]
$$

Equation (VI.68) is first subdivided into intervals in $\widetilde{E}$, each bounded either by an $\bar{E}_{k}$, or $\tilde{E}_{h}$ or $\tilde{E}_{h+1}$ :

$$
z_{h}^{\ell}(E)=\sum_{m=m_{\min }}^{m_{\max ^{-1}}} \int_{\tilde{E}_{m}}^{\tilde{E}_{m+1}} d \tilde{E} \pi^{l}(E+\tilde{E})\left[(1-W)+W E^{\prime}\right] .
$$

The indices ( ${ }^{\text {min }}$ ) and ( ${ }_{\text {max }}$ ) are such that:

$$
\begin{aligned}
& \text { 1. } \tilde{E}_{\mathrm{m}_{\text {Inin }}}=\tilde{\mathrm{E}}_{\mathrm{h}} \text { and } \\
& \text { 2. } \tilde{\mathrm{E}}_{\mathrm{m}_{\text {III }}}=\tilde{\mathrm{E}}_{\mathrm{n}+1} ;
\end{aligned}
$$



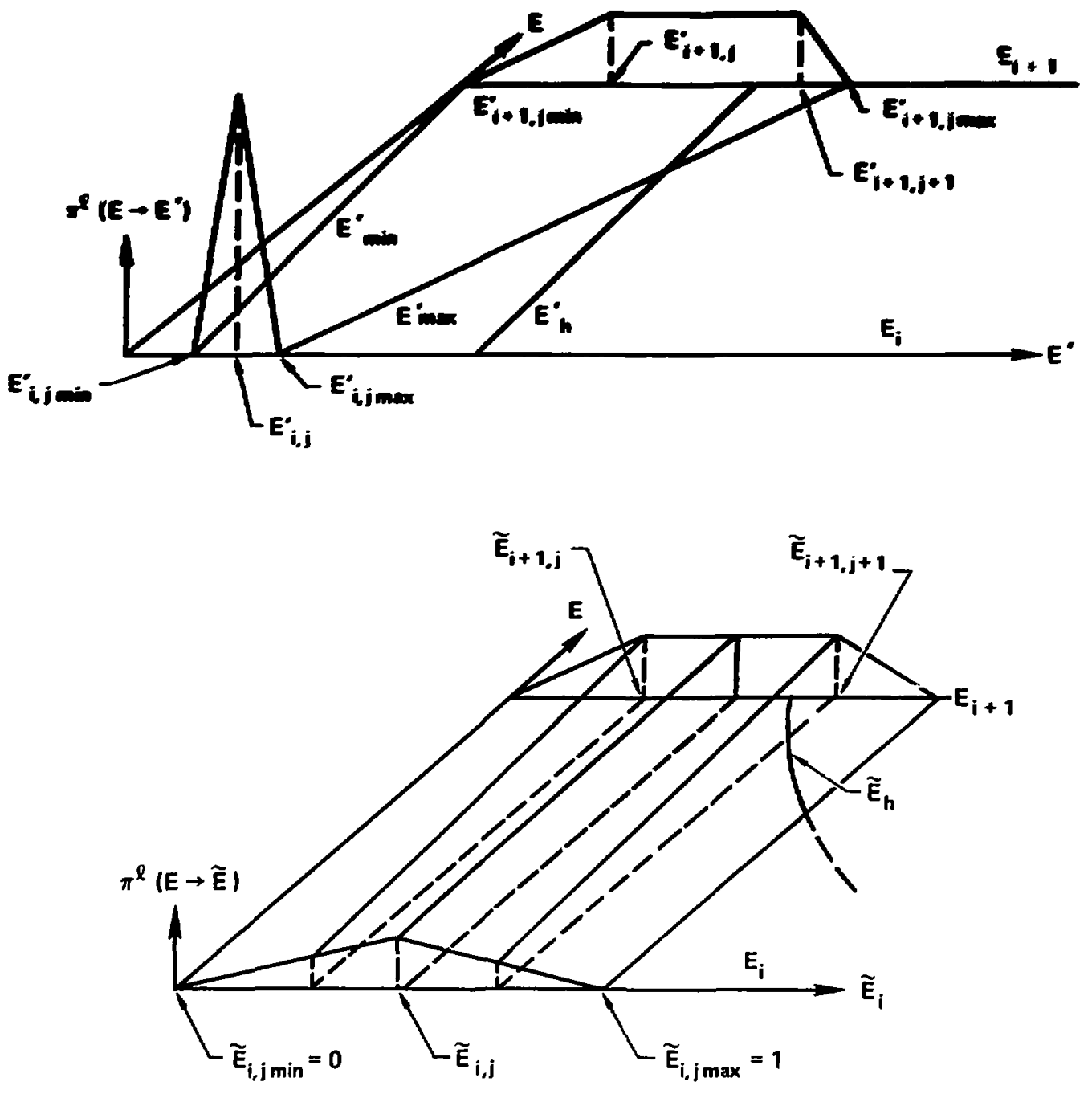

FIG. 2. A unit base transformation. Note that lines of constant $E^{\prime}$ (e.g., the complete set of $E_{h}^{i}$ ) do not necessarily transform to lines of constant $\widetilde{E}$. 


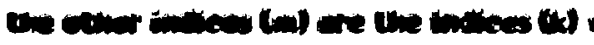

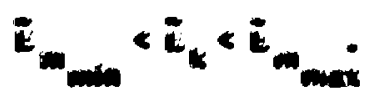

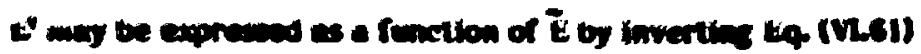

$$
t=A_{1}(\theta)+Y_{2}(\theta)
$$

(v.70)

whene

$$
A_{1}(E)=E_{\min }^{\prime}(E)
$$

and

$$
A_{2}(E)=E_{\max }^{\prime}(E)-E_{\min }^{\prime}(E) \text {. }
$$

Similarly from Eq. (Vl.67), the transformed probability inay be expressed as:

$$
\pi^{*}(E \rightarrow \tilde{E})=A_{J}(E)+A_{4}(E) \tilde{E} \text {, }
$$

where

$$
A_{4}(E)=\frac{\pi^{2}\left(E-\tilde{E}_{k+1}\right)-\pi^{l}\left(E-\tilde{E}_{k}\right)}{\tilde{E}_{k+1}-\tilde{E}_{k}}
$$

and

$$
A_{3}(E)=\pi^{\ell}\left(E+\tilde{E}_{k}\right)-A_{4}(E) \tilde{E}_{k} .
$$

The components of Eq. (VI.69) are integrated analytically:

$$
\begin{aligned}
& z_{h_{m}^{\ell}}^{\ell}(E)=\int_{\tilde{E}_{m}}^{\tilde{E}_{m+1}} d \tilde{E}\left[A_{5}(E)+A_{6}(E) \tilde{E}+A_{7}(E) \tilde{E}^{2}\right] \\
& =D_{m}(E)\left[A_{5}(E)+A_{6}(E) \overline{\tilde{E}}_{m}(E)+A_{7}(E) \overline{\tilde{E}}_{m}^{2}(E)\right],
\end{aligned}
$$

where

$$
\begin{aligned}
& A_{5}(E)=\left[(1-W)+W A_{1}(E)\right] A_{3}(E), \\
& A_{6}(E)=\left[(1-W)+W A_{1}(E)\right] A_{4}(E)+W A_{2}(E) A_{3}(E),
\end{aligned}
$$


$v_{m} m=z_{m+1}-E_{m}$

(nersed

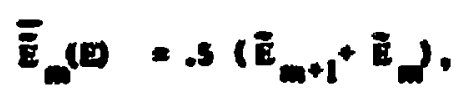

(virse)

and

$$
\tilde{E}^{2}(U)=\frac{1}{3}\left[\left(\tilde{E}_{m+1}\right)^{2}+E_{m+m} \tilde{E}_{m}+\left(\tilde{E}_{m}\right)^{2}\right)
$$

Equation (VL.75) expresues analytically the inner integration ,ver the $m^{\text {th }}$ interval in $\bar{E}$. All the component fectors, Egs. (VL.75), must be stated explicitly as functions of $E$, in anticipation of the outer integration operation. $E_{\min }^{\prime}(E)$ and $E_{n a x}^{\prime}(E)$ are evaluated by linearly interpolating between their respective

$$
\begin{aligned}
& \underbrace{\prime}_{\max }\left(E_{i}\right) \text { and } E_{\min }^{\prime}\left(E_{i+1}\right): \\
& E_{\min }^{\prime}(E)=b_{3}+b_{4} E
\end{aligned}
$$

and

$$
E_{\max }^{\prime}(E)=b_{1}+b_{2} E
$$

where

$$
\begin{aligned}
& b_{2}=\frac{E_{\max }^{\prime}\left(E_{i+1}\right)-E_{\text {max }}^{\prime}\left(E_{i}\right)}{E_{i+1}-E_{i}}, \\
& b_{1}=E_{\max }^{\prime}\left(E_{i}\right)-b_{2} E_{i}, \\
& b_{4}=\frac{E_{\min }^{\prime}\left(E_{i+1}\right)-E_{\min }^{\prime}\left(E_{i}\right)}{E_{i+1}-E_{i}},
\end{aligned}
$$

and 


$$
\theta_{j}=t_{\min }^{\prime}(t)-0, t_{j}
$$

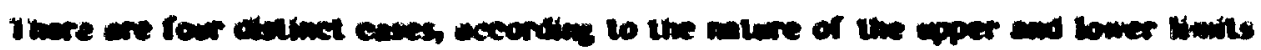

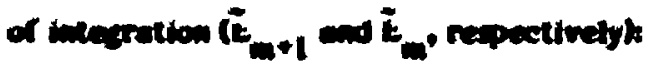

ciece U: $E_{n \rightarrow n}>E_{n}(t)$ and $E_{m+1}<E_{n+1}(t)$

Cere Le $E_{\text {on }}=E_{n}(t)$; and $\bar{E}_{m+1}<\bar{E}_{h+1}(E)$

Case K: $\quad \tilde{E}_{n}>\tilde{E}_{n}(E)$; and $\tilde{E}_{m+1}=\bar{E}_{n+1}(t)$

Case KL: $\tilde{E}_{m}=\tilde{E}_{h}(E)$; and $\tilde{E}_{m+1}=\tilde{E}_{h+1}(E)$

Equations (VI.75d, e, f) take the following form, depending on the case type:

\section{Case 0}

$U_{n i}(E): \tilde{E}_{m+1}-\tilde{E}_{m}$

$\overline{\tilde{E}}_{m}(E): .5\left(\tilde{E}_{m+1}+\tilde{E}_{m}\right)$

$\bar{E}_{m}^{2}(E): \frac{1}{3}\left[\left(\tilde{E}_{m+1}\right)^{2}+E_{m+1} \tilde{E}_{m}+\left(\tilde{E}_{m}\right)^{2}\right] \frac{b_{12}+b_{13} E+b_{14} E^{2}}{\left(b_{8}+b_{9} E\right)^{2}}$
(VI.78g)

$\mathrm{b}_{8}$ through $\mathrm{b}_{16}$ may be evaluated numerically for the various non-zero cases by using Eq. (VI.6I) and all of Eqs. (VI.77):

$$
\begin{aligned}
& b_{8}=b_{1}-b_{3} \\
& b_{9}=b_{2}-b_{4}
\end{aligned}
$$$$
\text { (all non-zero cases) }
$$ 


\section{ent}

\section{$0_{10} \frac{1}{2} E_{m+1}+b_{0}+\dot{E}_{n}-b_{j}$ \\ $\left.b_{11} \sum^{2} z_{(n+1} b_{2}-b_{3}\right)$}

$b_{12}=\frac{1}{3} \vec{E}_{m+1}^{2} b_{3}^{2}+E_{m+1} b_{s}\left(E_{h}^{\prime}-b_{3}\right)+\left(E_{h}^{\prime}-b_{3}\right)^{2}$

$b_{13}=\frac{1}{3}\left[2 \tilde{E}_{m+1}^{2} b_{8} b_{9}+\tilde{E}_{m+1}\left[\left(E_{h}^{\prime}-b_{3} b_{9}-b_{4} b_{8}\right]-2 b_{4}\left(E_{h}^{\prime}-b_{3}\right)\right]\right.$

$b_{14}=\frac{1}{3}\left(\tilde{E}_{m+1}^{2} b_{9}^{2}-\tilde{E}_{m+1} b_{4} b_{9}+b_{f}^{2}\right)$

$b_{15}=\tilde{E}_{m+1} b_{8}-\left(E_{h}^{\prime}-b_{3}\right)$

$b_{16}: \tilde{E}_{m+1} b_{9}+b_{4}$

Case R

$b_{10}: \frac{1}{2}\left(E_{h+1}^{\prime}-b_{3}+\tilde{E}_{m} o_{8}\right)$

$b_{11}: \frac{1}{2}\left(-b_{4}+\tilde{E}_{m} b_{g}\right)$

$b_{12}: \frac{1}{3}\left[\left(E_{h+1}^{\prime}-b_{3}\right)^{2}+\tilde{E}_{m} b_{8}\left(E_{h+1}^{\prime}-b_{3}\right)+\tilde{E}_{m}^{2} b_{8}^{2}\right]$ 


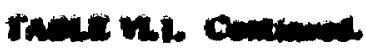

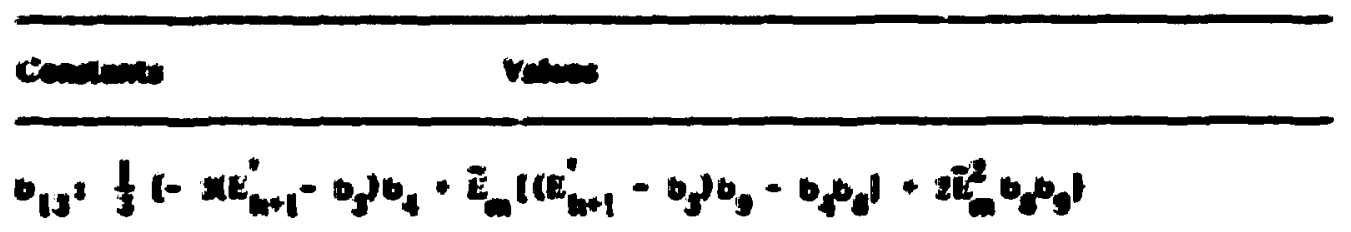

$$
\begin{aligned}
& b_{14}{ }^{2} b_{t}^{2}-\vec{E}_{m} b_{t} b_{y}+\vec{E}_{m}^{2} b_{j}^{2} \\
& b_{15}=\left(E_{h+1}^{0}-b_{3}\right)-\tilde{E}_{m} b_{3} \\
& b_{16}=-b_{4}-E_{m} b_{9}
\end{aligned}
$$

Case RL:

$$
b_{10}=\frac{1}{2}\left(E_{h}^{\prime}+\frac{E_{h+1}^{\prime}}{\left.-2 b_{3}\right)}\right.
$$

$$
\begin{aligned}
& b_{11}:-b_{4} \\
& b_{12}: \frac{1}{3}\left[\left(E_{h+1}^{\prime}-b_{3}\right)^{2}+\left(E_{h+1}^{\prime}-b_{3}\right)\left(E_{h}^{\prime}-b_{3}\right)+\left(E_{h}^{\prime}-b_{3}\right)^{2}\right] \\
& b_{13}: \frac{1}{3}\left[-2\left(E_{h+1}^{\prime}-b_{3}\right) b_{4}-b_{4}\left(E_{h+1}^{\prime}+E_{h}^{\prime}-2 b_{3}\right)-2\left(E_{h}^{\prime}-b_{3}\right) b_{4}\right] \\
& b_{14}: b_{4}^{2} \\
& b_{15}:\left(E_{h+1}^{\prime}-E_{h}^{\prime}\right) \\
& b_{16}: 0
\end{aligned}
$$

$$
\text { VI - } 27
$$




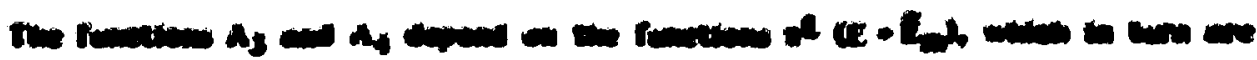

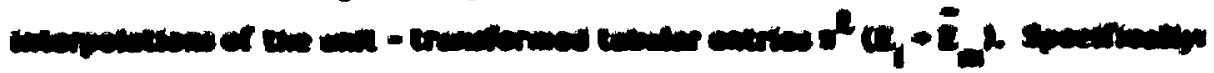

$$
\begin{aligned}
& -4 x+E x=0+400
\end{aligned}
$$

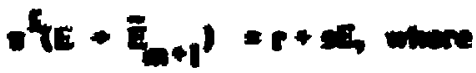

$$
\begin{aligned}
& q=\frac{\left.{ }^{2}\left(E_{1+1}+E_{1}\right)-\nabla^{4} E_{1}-E_{1}\right)}{E_{i+1}-E_{1}} \text {. } \\
& p=E^{2} E_{i}-E_{m i}-q E_{i} . \\
& s=\frac{\pi^{2}\left(E_{i+1}-E_{i n+1}\right)-\pi^{2}\left(E_{i}-E_{i n+1}\right)}{E_{i+1}-E_{i}} \text {, and } \\
& r=\pi^{l}\left(E_{i}+\tilde{E}_{m+1}\right)-s E_{i} \text {. }
\end{aligned}
$$

(IVT)

(ritewa)

Equations (VL80a, b and VL73a, b) lead to

$$
\begin{aligned}
& A_{4}(E)=b_{19}+b_{20} E \text {, and } \\
& A_{3}(E)=b_{21}+b_{22} E \text {, where } \\
& b_{19}=\frac{r-p}{\tilde{E}_{m+1}-\tilde{E}_{m}}, \\
& b_{20}=\frac{s-q}{\tilde{E}_{m+1}-\tilde{E}_{m}}, \\
& b_{21}=p-b_{19} \tilde{E}_{m}, \text { and } \\
& b_{22}=q-b_{20} \tilde{E}_{m} .
\end{aligned}
$$




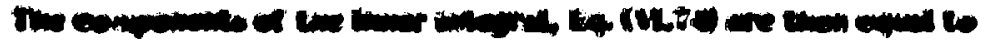

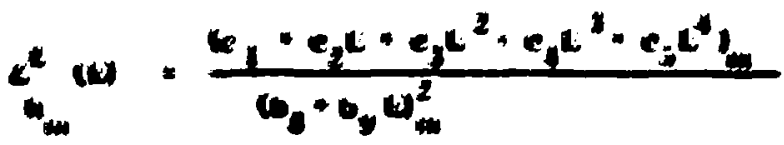

stesty

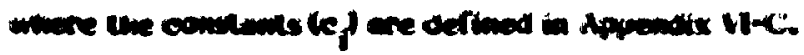

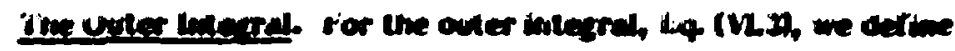

$d(t)=1+u t$.

(VLen)

$\boldsymbol{p}(\mathrm{t})=v+w t$

(V)esto

$M(t)=B+h i$

(VL8te)

The outer integral, over a given inner subintegral $m$, using Eqs. (VL.3), (VL.83), and (VL84) becomes

$$
J_{g-h}(m)=\int d E \frac{(t+u E)(v+w E)(g+h E)\left(c_{1}+c_{2} E+c_{3} E^{2}+c_{4} E^{3}+c_{5} E^{4}\right)}{\left(b_{8}+b_{9} E\right)_{m}^{2}}
$$

A word about the outer integration limits: As with the inner integral, it must be subdivided at breakpoints determined by the data entry locations of $\alpha E), \phi^{l}(E)$, or $m(E)$, and $\pi^{\ell}\left(t+t^{\prime}\right)$. In addition, there are breakpoints in the outer integral where the case type of the inner integral changes. That is, whenever $\tilde{E}_{h}$ crosses $E_{n_{m i n}}$ or whenever $\tilde{E}_{h+l}$ crosses $E_{\text {fit } \max }$. Tak outer integral is thus a double sum of subintegrals in $E$ and $E$ :

$$
J_{\ell_{g}+h}=\sum_{\min }^{n_{\max }} \sum_{\min }^{-1} \mathrm{~J}_{\ell_{g+h}}^{(m, n)},
$$

where each $\mathrm{J}\left(\mathrm{In}_{\mathrm{Q}_{\mathrm{g}+h}}\right.$ has the form Eq. (V1.85)

$$
J(\pi, n)_{\ell \rightarrow+1}=\int_{E_{n}}^{E_{n+1}} d E \sum_{i=1}^{i} \frac{d_{i} E^{j-1}}{\left(b_{8}+b_{9} E\right)^{2}} .
$$




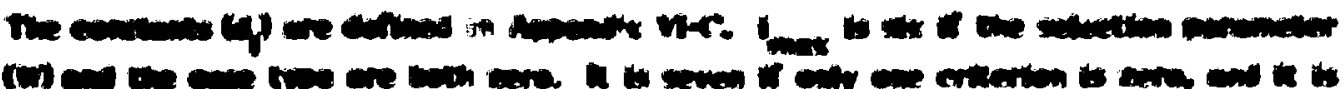

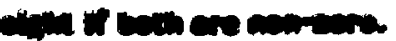

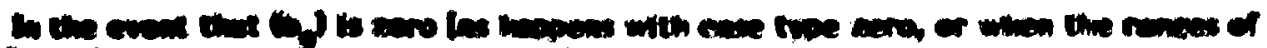

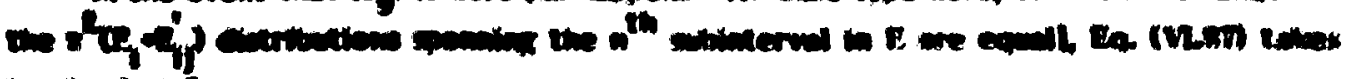
tes siminiont forms

$$
\min _{t \rightarrow \infty}=\frac{1}{b} \sum_{i=1}^{\prime} \frac{d_{1}\left(E_{n+1}^{i}-E_{n}^{\prime}\right)}{t}
$$

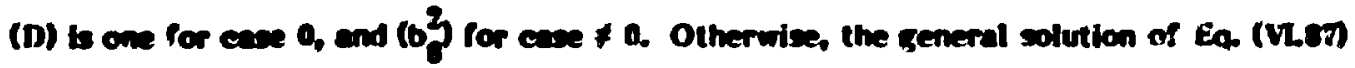
is

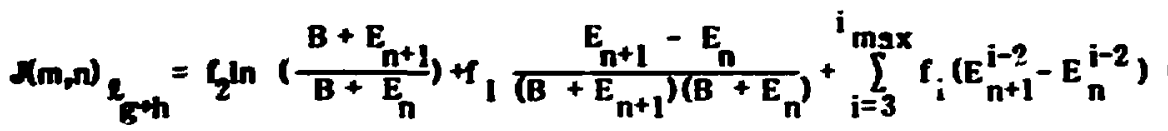

$B$ is the ratio $b_{8} / b_{g}$, and the $f_{i}$ are defined in Appendix $v \mathrm{I}-\mathrm{C}$.

It is important to note that in order to minimize computing roisndoff error in a finite bit mechine, close attention must be paid to factoring out difference terms where possible. $\left(E_{n+1}-E_{n}\right)$ is an obvious example. Using this philosophy, TABXFR requires only single precision to obtain results with negligible error.

\section{Energy Production and Deposition}

For each target isotope under considerution, CLYDE obtains various rate coefficients which pertain to energy conservation. Specifically, these items are total available energy, energy production, and the energy in the outgoing particle field. These data allow a user to caleulate, within his transport code, the energy production as well as the local energy deposition which is specific to the choice of particles being transported. ${ }^{2}$

When CLYDE is used to calculate multigroup constants for identical incident and outgoing particles, e.g., $y_{0}=y_{i}$, it computes an average energy of the incident particle $y_{i}$ entering into the reaction

$$
\langle E \sigma\rangle_{g}=\sum_{\mathbf{r}} \frac{\int_{g} E \sigma_{r}(E) \varphi^{\circ}(E) d E}{\int_{g} \varphi^{0}(E) d E},
$$




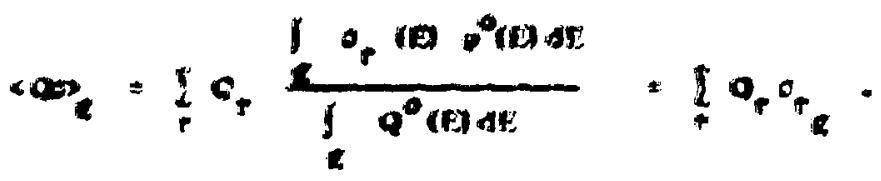

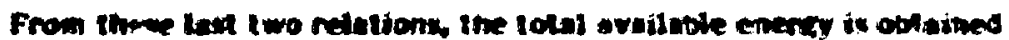

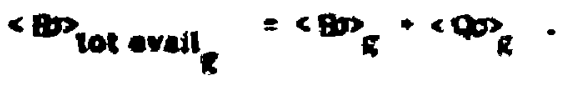

For all CLYDE malculations (independent of whether or not $w_{0}=y_{j}$, , itwe code computes the total s rerage energy in the oulgoing narticle field for the specified $v$,

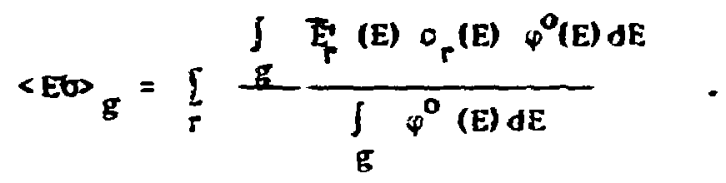

In Eg. (VL.93, $\bar{E}_{\mathbf{r}}^{\prime}(E)$ is the total average energy to the $y_{0}$ particle field induced by reaction r. This term is precalculated by ENDEP (see Chapter III) and stored in the evaluated library for each reaction and outgoing particle as $I=10$ type data.

With the above rate coefficients, the local energy deposition may be programmed, at transport code time, to be the total available energy less the sum only of the total average energy in the outgoing particle field of those paricles to be specifically transported. ${ }^{2}$

\section{Isotope Production}

CLYDE lists, with each reaction, a set of data pairs, which list all secondary particles, including residual nuclei, and their multiplicities. There are two exceptions: neutrons resulting from fission reactions are listed with "zero" multiplicity, and gamma ray secondaries are not listed at all. The multiplicities in these two situations depend on the initial particle energies, and are consequently made implicit in the respective transfcr matrices for these particles.

D. INPUT, OPERATION, AND OUTPUT

The format of the ASCII input file for CLYDE is described in Table VI.2.

$$
V I-31
$$




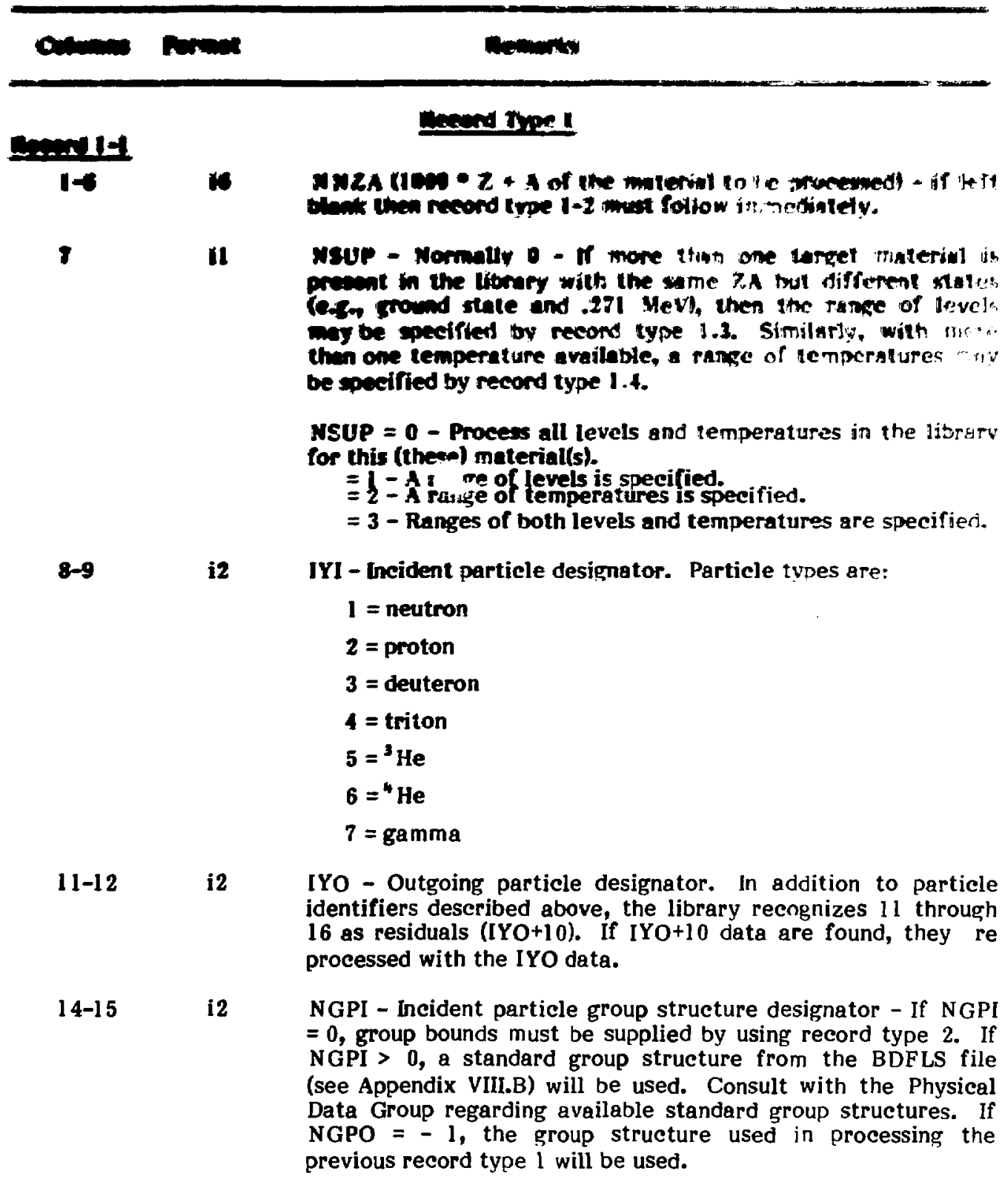




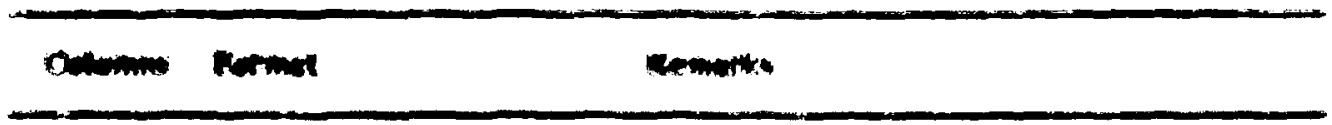

1748

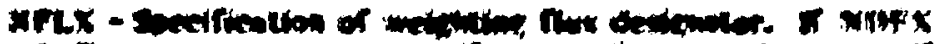

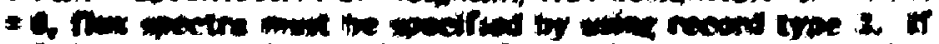

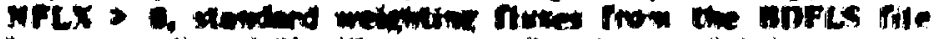

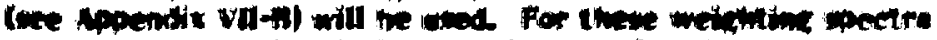

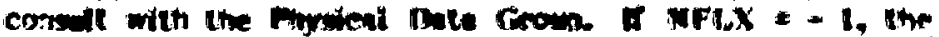

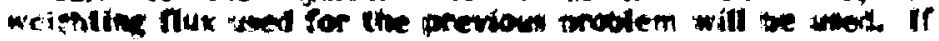

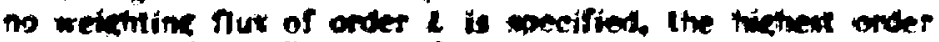
thet is epecified will the nawed for order $k$.

21

a

29-30

33

36

il

$38-45$

$47-48$

ItCFLS: - Controls the enerty/parlicle conservation metion to be used by CLYYUE:

=0 - particle conscivation

$=1$ - enerty production conservation

$=2$ - energy conservation

$=3$ - group splitting.

Regardless of input, if the outgoing particle is a neutron, IECFLG is set to 0. It should also be 0 for gamma in - gemma out, otherwise I for gamma out. It should be 3 for outgoing charged particles.

i2 NPNTOP - controls the amounty of hsp output and is normally 0. If NPNTOP $=2$, energy deposits and transfer matrix contributions will be output to hsp as they are calculated for each reaction. Values of NPNTOP less than 0 , or greater than 2, are used only for debug purposes, and result in much output.

il NOUTOP - If NOUTOP $=0$, only hsp output will result. If NOUTOP $=1$, output will go in an appropriate format to the output file specified on the execute line.

NOUT - If NOUT $=0$, NPNTOP is set to 2, and all reactions defined for a material in the library will be processed, but no total transfer matrix or fission matrix will be output. If NOUT $=1$, the appropriate reactions will be processed for a transport/diffusion code. Fission is output as a fission matrix separate from the transfer matrix, but is also added into the total transfer matrix.

EPIA - Specifies the relative order of accuracy desired in doing numerical integrations. EPIA $=.0001$ produces good results without using excessive machine time.

LLMAX - specifies the maximum order of transfer matrice, L, to be calculated. The code calculates (LLMAX + 1) matrices and uses the LLMAX + lst matrix to form a transport correstion. If $\mathrm{LLMAX}=-1$, then the code calculates only group cross-sections and energy deposits. 


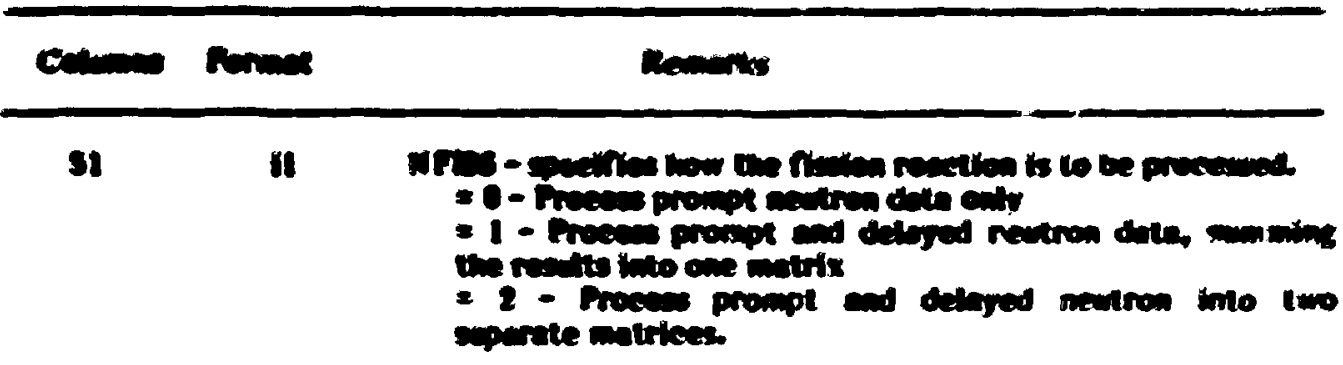

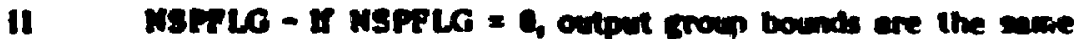

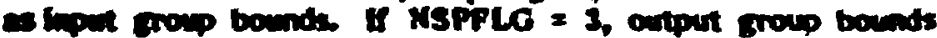
are not the sane as input group bounds, see reeond I-5 for speciflention of output group bound. if NSPFLG $=6$, Use outpot group bounds from previous problem.

II NTCFLG specifies the transport correction to be used. If 0<NTCPLG<3, the transport correction term is included in the total transfer matrix. If $4<N T C F L G<7$, all transport correction terms up through order TLMAX+1 are included in a separate array. This latter form is the required input for NDPL (see Chapter VI). If NTCFLG $=0$ or 4 , the code decides. If NTCFLG $=1$ or 5 , an approximate correction will be used. If NTCFLG $=2$ or 6 , the exact correction will be used. If NTCFLG $=3$ or 7 , no correction will be used.

71

72

76-80 il

IVFLG multiplies $\sigma_{x}(E)$ by velocity $V(E)$, so that CLYDE produces $\left(\sigma_{x} \nu\right)_{g}$ rather than $\sigma_{x g}$.

il NLAST is the last problem flag. If NLAST $=0$, another problem is to follow. If NLAST $=1$, no more problems follow.

i5 IWALT is a selective output sentinal. It allows suppression of output to the output ASCIl file but not to the hsp file. Since IWALT may be changed internally during the calculation, this sentinal should not be used without consulting the Physical Data Group. Each type of output has associated with it an ID. To get all output leave IWALT blank, but to get selected output calculate IWALT as follows:

\begin{tabular}{lll} 
ID & \multicolumn{1}{c}{ Output Item } & $\underline{2^{\text {ID }-1}}$ \\
1 & Group bounds & 1 \\
2 & Speed & 2 \\
3 & Group flux & 4 \\
4 & Group cross section & 8 \\
5 & Energy available & 16 \\
6 & Energy production & 32
\end{tabular}




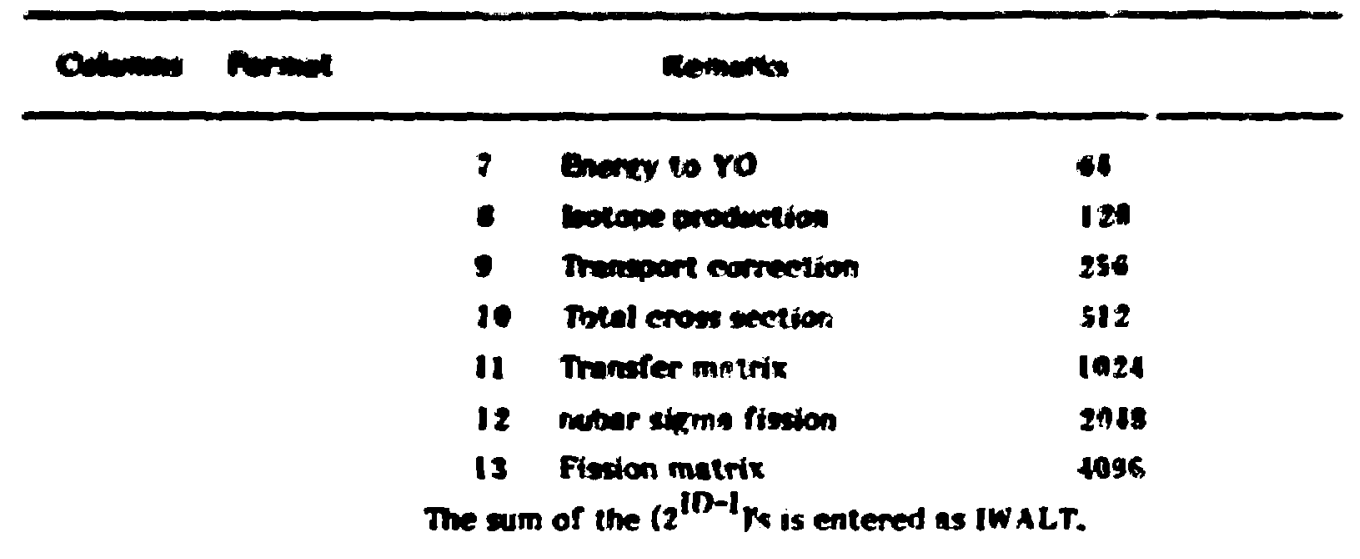

Recond 1-2 Range of materials (Needed only if :INZA of reeord I is blank)

1-6 i6 N NZAB begining material

$=1000 * Z+A$ for the first material

7-1: $\quad$ i6 N NZAE ending material

$=1000 * Z+A$ for the last material

Record 1-3

Needed only if NSUP $=1$ or 3

$1-12$

E12.5 ELVB least energy of a level in a target material.

13-24 El2.5 ELVE greatest energy of a level in a target material.

Record 1-4 Needed only if NSCP $=2$ or 3

1-12 $\quad$ BL2.5 TEMPB least material temperature.

13-24 E12.5 TEMPE greatest material temperature.

Record 1-5

$1-3$

i3

Needed only if NSPFLG $=3$

NGPO - Outgoing particle group strueture designator. If NGPO $=0$, the user supplies the group structure using record type 2. If NGPO > 0 , a standard group structure from the BDFLS file (see Appendix VII-B) will be used. Consult with the Physical Data Group for information about standard group structures.

\section{Record Type 2}

Record 2-1

$1-4$

Needed only if NGPI or NGPO is 0

NEBI or NEBO - number of energy boundaries to be read from record 2-2. Number of bounds $=$ number of groups +1 . 


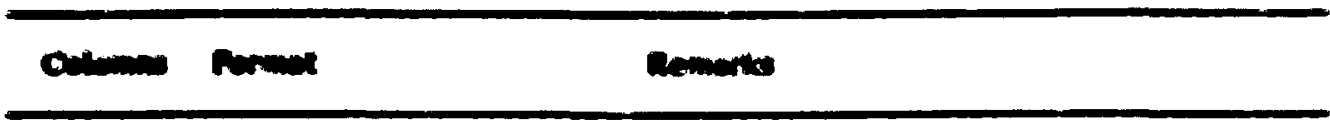

Land 20

$1-12$

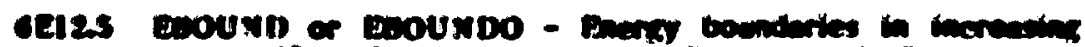

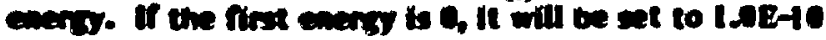

11-72

Record ispe 2 for input bounds must preceed record type 2 for output bounds if both are beils supplied.

Recond 3-1

$1-4$

Record 3-2

$1-4$

14

14

Needed only if NPLX $=0$

LFLUX is the highest order, L, for which fluxes are supplied.

LFLUX + 1 records are required; one for each $\ell$

NFLUX( $(l)$ number of entries to be read from record 3-3 for $\ell=0$ (i.e., 2 times number of $E, \phi$ pairs)

NFLUX for all orders of $\ell$ must preceed records 3-3.

Record 3-3

1-12

$6 \mathrm{E} 12.5$

$E, \phi$ pairs - start new record for each $\ell$ order

EFLUX - Energy flux pairs in increasing energy. Energies must span the group bounds used for the problem. If the problem is to be run to a higher Legendre expansion order than that for which fluxes are provided, the highest order fluxes available are used for all higher orders.

Records 3-3 are supplied as sets for each L-order.

The calling sequence for CLYDE is the following:

$$
\text { omega clyde (input file) (box no.) (hsp option) }
$$

(library name) 0 (output file name) (output file size) / T V 
thene the

ton to.

his epition

library name

$\mathbf{0}$

output file name

output file size

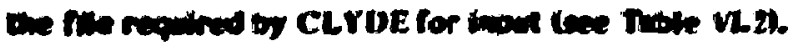

rarly bou number

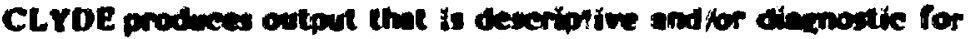
the povitem. The deneriptive iaforention is dependent on the thpe of output called for and is contatined in the family of files

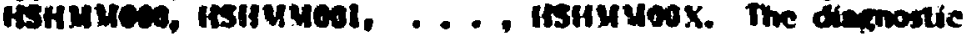
informatlon contuins the inpul calling sequence and the ru cecription, and is in the flle PRT/IIMH. The numbers HMN are the three rifst hand numbers of the machine Renerated time (24) hour scale) e-6o, if the time the problem was generated was 0431 or 1431. then HMN $=$ 431. This output fany be direeted to the PR80 (use "Ir80" for option) , the hiph speed printer (use Tisp" for option), or keot for inspection (use "debur" for aption) and/or output to RJET or other medium using ALLOUT.

the evaluated nuclear data file, e.g., ENDL file.

The zero in this position indicates that the library exists as a file. The zero must be included in the calling sequence.

the name of the CLYDE ASCII file to be ereated as output.

If equal or less than 99 , it is the size of the file to be created in units of 100000 . If greater than 99 , it is the actual file size to be created. When the code finishes it will shrink the file to the size needed.

E. CLYDE OUTPUT FORMAT AND INSTRUCTIONS

The format of the ASCII output file for CLYDE is described in Table VI.3.

TABLE VI.3. CLYDE Output (Code Usable ASCII File).

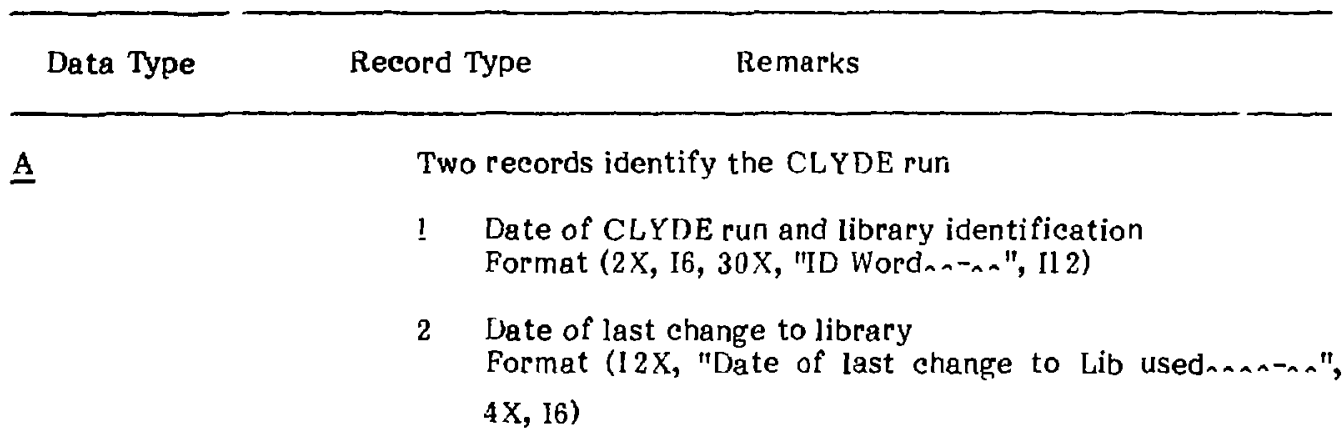

$$
V I-37
$$




\begin{tabular}{|c|c|c|}
\hline Deta tyee & Deteod thowe & Remartus \\
\hline
\end{tabular}

bots bpes relete to matertal anth. For ench material there are one or two

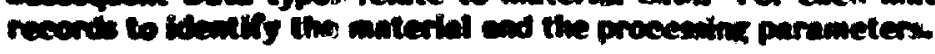

B

$\underline{\mathbf{c}}$

$\underline{\mathbf{D}}$

$\underline{\mathbf{E}}$

$\underline{E_{2}} \mathrm{D}=1 \mathrm{CP}$ BDS

$E, 1 \mathrm{D}=2$ Speed

$\underline{E}, \mathrm{ID}=3$ GP FLUX

$\underline{E}, I D=4$ GP X-Sect

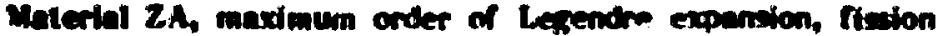
seativeh, Ispe ealeculation done, number of output sroups, nmaber of input groups, transport correetion tvpe, number of reactions, number of particles produced, input group structure ID No, hux speetrum In No, conservation semtine!, and output sroup structure ID Na. Format (16, 1513, 20X, "*n)

Conditional - If the transport correction nag is more than 3, then output incident and outgoing particle identifiers, material exeitation level, material temperature, atomic mass, and date of last change.

Format (212, 3E11.4, 1X, I6)

Conditional - If the incident and outgoing $n$ rticles are the same, then output a list of the reaction , ifiers for all reactions processed.

Format (2413)

Miay be selectively suppressed by user or by code. The following data consist of two or more records, the first of which identifies the tvpe of data following, and has a format of the form (14, I4, 1X, A8). The second variable is sometimes unused.

1 Data type ID, Group structure ID, and data type.

Format ("^^^1", 14, 1X, "GP^BDS^॰")

2* Group boundaries. (NEBI values, 6E1 2.5)**

1 Data type ID and data type.

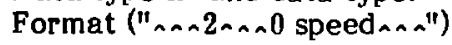

2* Group speeds. (N EGI values, 6E12.5)***

1 Data type ID, Flux structure ID, and data type. Format ("^^^3", I4, 1X, "GP^Flux ^")

2* Group fluxes. (N EGI values, 6E12.5)

1 Data type ID and data type.

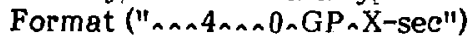




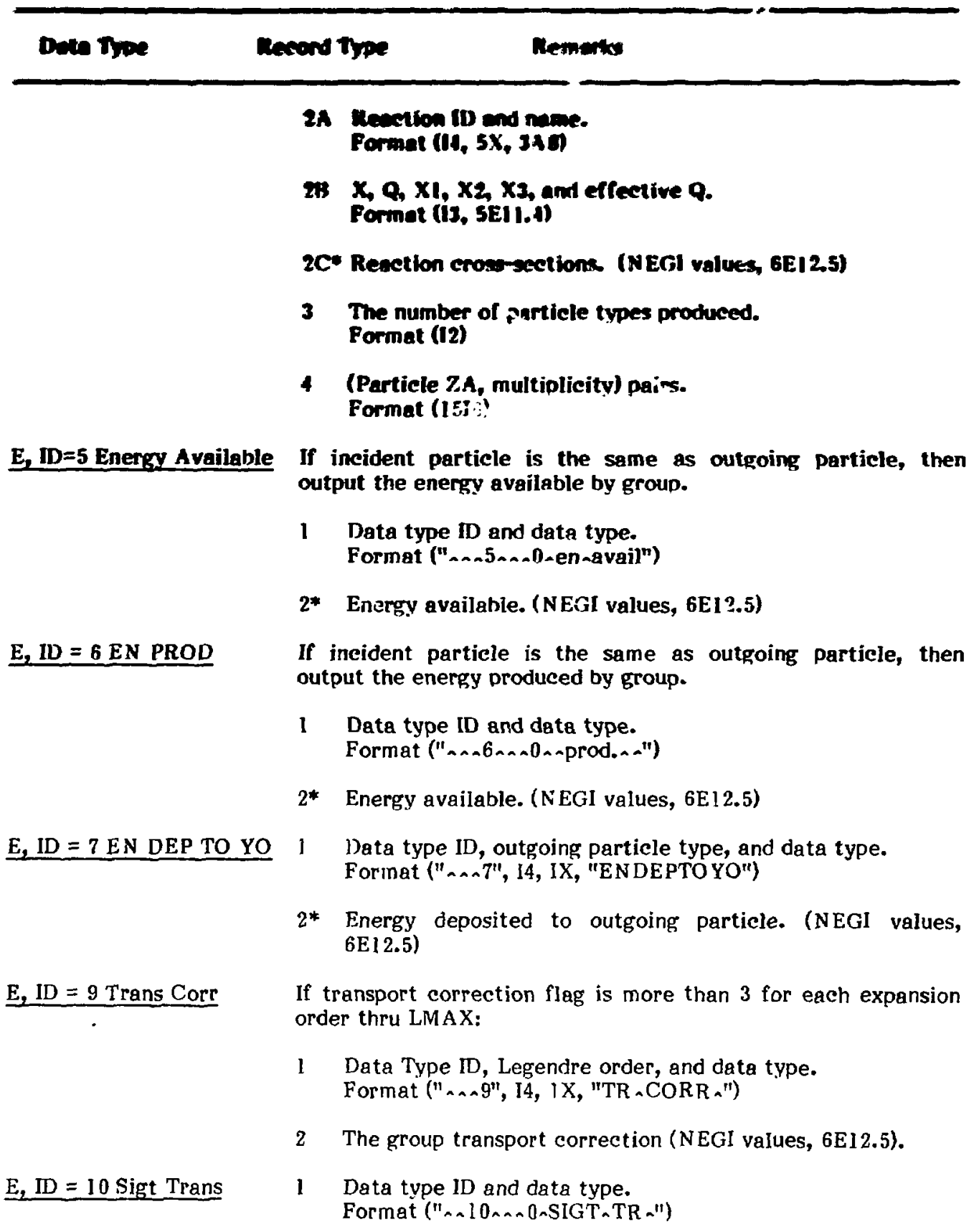




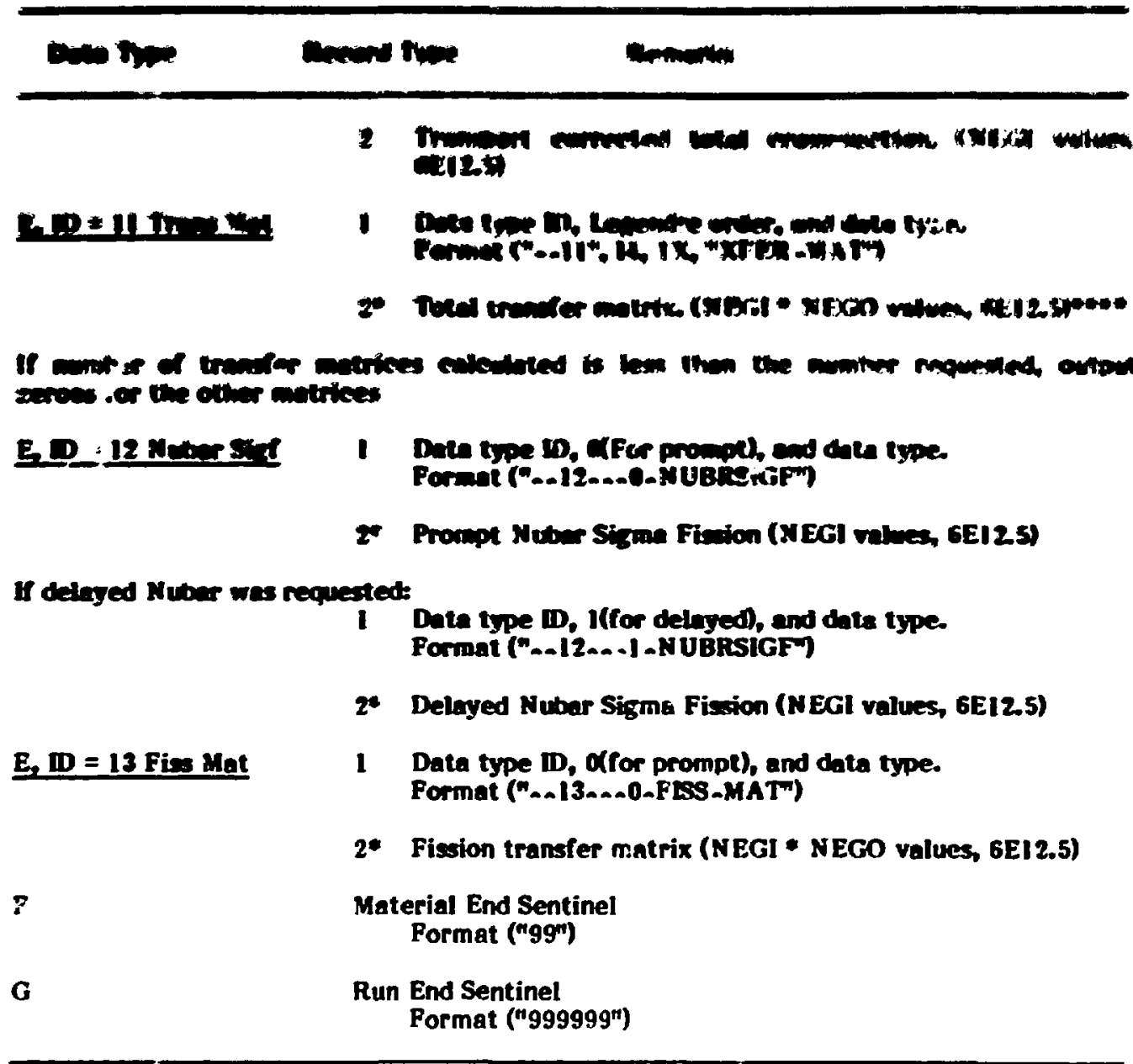

Note: The symbol $a$ in the table signifies a blank.

* As many repetitions of the record as required

* $\quad$ NEBI - - Number of energy bounds = NEGI + 1 III

*** NEGI - - Number of incident groups

**** NEGO - - Nuriber of emission groups 


\section{APPENOX $\mathbf{n - \lambda}$}

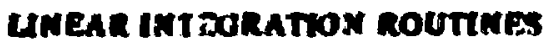

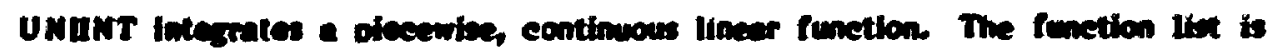

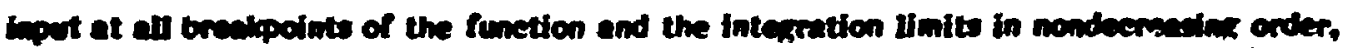
and containe the values of the integrand at those points. The operation perfomed is

$$
\int_{x\left(K_{1}\right)}^{x\left(K_{2}\right)} d x F=\sum_{k=K_{1}}^{K_{2}-1} \frac{1}{2}[x(K+1)-x(K)][F(K+1)+P(K)] .
$$

where $K_{1}, K_{2}$ define the limits of integration over the variable $x$ and function $F$.

\section{DUNT}

DINT integrates the product of two piecewise, continuous linear functions. ${ }^{8}$ The variable of integration is listed at the integration limits and at the breakpoints of both functions; the two function lists contain the values of the functions at these entries of the variable list:

$$
\begin{gathered}
\int_{x\left(K_{1}\right)}^{x\left(K_{2}\right)} d x F \cdot G=\frac{1}{6} \sum_{K=K_{1}}^{K}[x(K+1)-x(K)]\{[F(K+1)+F(K)] \\
\cdot[G(K+1)+G(K)]+F(K) \cdot G(K)+F(K+1) G(K+1)\},
\end{gathered}
$$

where $F, G$ are the functions and $K_{1}, K_{2}$ the integration limits.

\section{TRIINT}

TRIINT integrates the product of three piecewise continuous linear functions, ${ }^{8}$ of the same form as in DIINT, above. The equation is

$$
\begin{aligned}
\int_{x\left(K_{1}\right)}^{x\left(K_{2}\right)} d x F \cdot G \cdot H=\frac{1}{12} \sum_{K=K}^{K}[x(K+1)-x(K)] \\
\cdot\{[2 F(K) \cdot G(K) \cdot H(K)+F(K+1) \cdot G(K+1) \cdot H(K+1) \\
+[F(K)+F(K+1)][G(K)+G(K+1)][H(K)+H(K+1)]\} .
\end{aligned}
$$




\section{APreadix $n-1$ \\ wurearchl ixtegention}

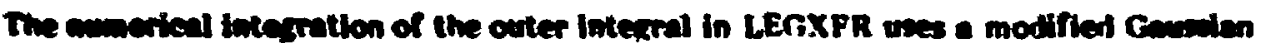
Integration scheme dewtind by Cles. This procedure is more economical then the

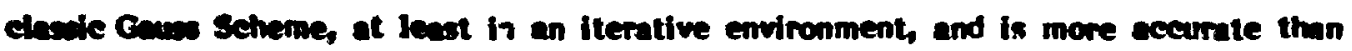
Simpsorts rule. Strietly speaking, Simpsorfs rule is only 3rd order accurate, with the megnitude of the th crder term diminishing as $2-\frac{64}{15}(M-1)^{-1}$. I(M) is the numher of meavirements, or subcutine entries, macie in "he interval of integration, as it is subeanged.) Gaus integration, on the other tha. is accurate to order $2 \mathrm{~N}-1$ of an expantion in Legendre polynomiais. The Modified Gauss scheme is accurate to orrer $M$ of a power series expansion.

When iterating, however, the Gaussian integration, so as to advance to the next bigher order, requires a complete new set of arguments. A figure of merit, the ratio of order of accuracy, to the cumulative number of arguments required to arrive at that accuracy is:

$$
S=\frac{2(21+1)}{(I+1)^{2}+1}
$$

where $I$ is the iteration number. It is clear that as more iterations are required the "cost effectiveness" goes down. The Modified Gauss system uses only two new arguments with each iteration, so that its figure of merit is always one independent of (I).

In the modified Gauss system, the function being integrated is represented by a power series in the interval $(-1,1)$.

$$
F=\sum_{m=0}^{\infty} a_{m} r^{m}
$$

its integral is $J=\int_{-1}^{1} F d r=2 \sum_{m=0}^{\infty} \frac{a}{2 m+1}$.

Consider a pair of measurements at arguments $\left( \pm r_{i}\right)$; their sum is

$$
F_{i}=F\left(r_{i}\right)+F\left(-r_{i}\right)=2 \sum_{m=0}^{\infty} a_{2 m} r_{i}^{2 m}
$$


$\sin t^{t h}$

$$
J_{1}=\sum_{i=0} a_{1, j} a_{1}=2 \sum_{i=0} a_{b, j} \sum_{m=0} a_{2 m} r_{i}^{2 m}
$$

\section{(J) will epperodianate the integreal $(J)$ to onder of secumby $(21+1)$, if}

$$
\begin{aligned}
& J=J_{1} \\
& 2 \sum_{m=0}^{1} \frac{a_{2 m}}{2 m+1}=2 \sum_{m=0} a_{2 m} \sum_{i=0}^{I} \alpha_{i, I} r_{i}^{2 m} ;
\end{aligned}
$$

\section{leading to a set of $(1+1)$ equations of the form}

$$
\frac{1}{2 m+1}=\sum_{j=0} \alpha_{i, I} r_{i}^{2 m} \quad 0 \leq m \leq I
$$

The first two argument sets, used in the Modified Gauss scheme are legislated to be $r_{0}^{2}=0$ and $r_{1}^{2}=1$, so as to be able to test early in the iterative process for an empty interval or for a highly peaked funetion. Subsequent values of $r_{i}^{2}$ are chosen from the interval (0,1). Starting then, with $i=2$, the Eqs. (VI-B.4) are solved by machine, using trial $r_{i}$ 's, for the corresponding set of $\alpha_{i, i} s$. That $r_{i}$ is chosen for which its value of

$$
\sum_{i=0}^{1}\left(\alpha_{i, I}\right)^{2}
$$

is minimized. This eriterion is used so as to reduce round-off error in computing Eq. (VLC-3) as much as possible. As each $\left(r_{i}\right)$ is chosen, a choice is again made for an $\left(r_{i+1}\right)$ and the process is repeated. In this way a set of $\left(R_{i}\right)$ and their attendant coefficients $\left(\alpha_{i, I}\right)$ are precalculated, and stored as program constants in LEGXFR.

When LEGXFR attempts an integration iteratively, two new functions are calculated at anguments $r_{I}$ and $-r_{I}$ by subroutine entry. These new functions, along with the previously determined set at $\pm r_{i<1}$ are linearly combined, using the coefficients $\alpha_{i, 1}$, to give a new estimate of the integral $\mathrm{J}_{\Gamma}$ when $\mathrm{J}_{\mathrm{I}}$ is satisfactorily close to $\mathrm{J}_{\mathrm{I}-\mathrm{l}}$, the integration is said to have converged, and the process is terminated. The convergence criterion is an input parameter. 


\section{APPENOAX n-C}

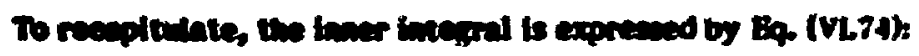

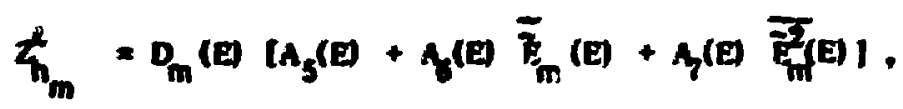

(VL74)

where

$$
\begin{aligned}
& A_{5}(E)=\left[(1-W)+W A_{1}(E)\right] A_{3}(E) \\
& A_{6}(E)=\left[(I-W)+W A_{1}(E)\right] A_{4}(E)+W A_{2}(E) A_{3}(E),
\end{aligned}
$$

(VL75a)

(VI.75b)

and $A_{7}(E)=W A_{2}(E) A_{4}(E)$.

(VL75C)

Furthermore $A_{1}(E)=b_{3}+b_{4} E$, from Eqs. (VI.71a) and (V.76a), $A_{2}(E)=b_{8}+b_{9} E_{7}$ from Eqs. (VL.7lb), (VI.76a), and (VL.76b); elso,

$$
A_{3}(E)=b_{21}+b_{22} E
$$

and $A_{4}(E)=b_{19}+b_{20} E$,

and finally,

$$
\begin{array}{ll}
\text { Case 0 } & \frac{\text { Case } \neq 0}{b_{15}+b_{16} E} \\
D_{m}(E)=b_{15}(E) & \frac{b_{10}+b_{11} E}{A_{2}(E)} \\
\overline{\tilde{E}}_{m}(E)=b_{10} & \frac{b_{12}+b_{13} E+b_{14} E^{2}}{A_{2}^{2}(E)}
\end{array}
$$

Distinguishing between case 0 and case $\neq 0$, we express Eg. (VI.74) in terms of the numerical constants $b$ and powers of $E$ : 


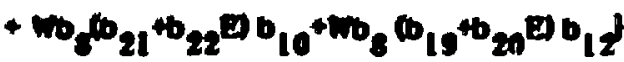

(v-c.l)

\section{creste}

$$
\begin{aligned}
& f_{h_{m}}^{l}=\frac{b_{15}+b_{18} E}{\left(b_{8}+b_{9} E\right)^{2}}\left\{\left(\left(1-w+w b_{3}\right)+w b_{q} E\left(b_{2 !}+b_{22} E\right)\left(b_{8}+b_{g} E\right)\right.\right. \\
&+\left[\left(1-w+w b_{3}\right)+\left(w b_{4} E\right]\left(b_{19}+b_{20} E\right)\left(b_{10}+b_{11} E\right)+w\left(b_{8}+b_{9} E\right)\left(b_{21}+b_{22} E\right)\left(b_{10}+b_{11} E\right)\right. \\
&\left.+w\left(b_{19}+b_{20} E\right)\left(b_{12}+b_{13} E+b_{14} E^{2}\right)\right] \\
& \text { (V1-c.2) }
\end{aligned}
$$

The evolution of the succeeding constants into the constants $c_{1}$ through $c_{5}$ follows the programming in the subroutine EINTG. For Case 0:

$$
\begin{aligned}
& \mathrm{Cl}_{2}=\mathrm{b}_{15}\left(\mathrm{~b}_{21}+\mathrm{b}_{19} \mathrm{~b}_{10}\right) \\
& \mathrm{Cl}_{3}=\mathrm{b}_{15}\left(\mathrm{~b}_{22}+\mathrm{b}_{20} \mathrm{~b}_{10}\right)
\end{aligned}
$$

and

$$
\mathrm{CI}_{1}=\mathrm{Cl}_{4}=\mathrm{CI}_{5}=\mathrm{CI}_{6}=0
$$

In the event the selection parameter $W$ is zero, the constants CC are all zero. Otherwise,

$$
\begin{aligned}
& \mathrm{CC}_{2}=\mathrm{b}_{8}\left(\mathrm{~b}_{21} \mathrm{~b}_{10}+\mathrm{b}_{19} \mathrm{~b}_{12}\right) \\
& \mathrm{CC}_{3}=\mathrm{b}_{8}\left(\mathrm{~b}_{22} \mathrm{~b}_{10}+\mathrm{b}_{20} \mathrm{~b}_{12}\right) .
\end{aligned}
$$

For Case $\neq 0$ :

$$
\begin{aligned}
& c_{7}=b_{8} b_{21}+b_{10} b_{19} \\
& c_{8}=b_{9} b_{21}+b_{10} b_{20}+b_{8} b_{22}+b_{11} b_{19} \\
& c_{9}=b_{9} b_{22}+b_{11} b_{20}
\end{aligned}
$$




$$
\begin{aligned}
& a_{2}=b_{15} c_{7} \\
& a_{3}=b_{16} c_{7}+b_{15} c_{5} \\
& a_{4}=b_{10} c_{8}+b_{15} c_{9} \\
& c_{3}=b_{16} c_{9}
\end{aligned}
$$

Again, if the selection parameter (iV) is zero, all constants (CC) are zeru. Otherwise

$$
\begin{aligned}
& c_{10}=b_{8} b_{22}+b_{9} b_{21} \\
& c_{11}=b_{12} b_{20}+b_{13} b_{19} \\
& c_{12}=b_{14} b_{19}+b_{13} b_{20} \\
& c c_{2}=b_{8} b_{10} b_{21}+b_{12} b_{19} \\
& c c_{3}=b_{10} b_{11} c_{10}+b_{11} b_{8} b_{21}+c_{11} \\
& c c_{4}=b_{11} c_{10}+b_{10} b_{9} b_{22}+c_{12}
\end{aligned}
$$

(VI-C.7a)

(VI-C.7b)

Finally, for all cases, if the selection parameter $W$ is zero:

$$
c_{i}=\mathrm{Cl}_{\mathrm{i}+1}
$$

If it is not, then

$$
\begin{aligned}
& c_{1}=b_{3} * \mathrm{Cl}_{2}+b_{15} * \mathrm{CC}_{2} \\
& c_{2}=b_{3} * \mathrm{Cl}_{3}+\mathrm{b}_{4} * \mathrm{Cl}_{2}+\mathrm{b}_{15} * \mathrm{CC}_{3}+\mathrm{b}_{16} * \mathrm{CC}_{2} \\
& c_{3}=\mathrm{b}_{3} * \mathrm{Cl}_{4}+\mathrm{b}_{4} * \mathrm{Cl}_{3}+\mathrm{b}_{15} * \mathrm{CC}_{4}+\mathrm{b}_{16} * \mathrm{CC}_{3} \\
& c_{4}=\mathrm{b}_{3} * \mathrm{Cl}_{5}+\mathrm{b}_{4} * \mathrm{Cl}_{4}+\mathrm{b}_{15} * \mathrm{CC}_{5}+\mathrm{b}_{16} * \mathrm{CC}_{4} \\
& c_{5}=\mathrm{b}_{4} * \mathrm{Cl}_{5}+\mathrm{b}_{16} * \mathrm{CC}_{5}
\end{aligned}
$$

These are constants in Eq. (VI.83). 


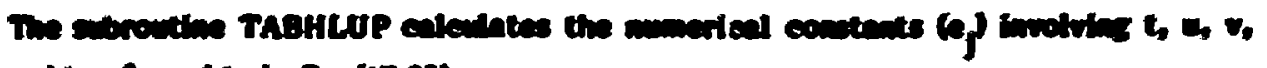

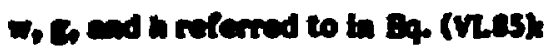

$$
\begin{aligned}
& e_{1}=w \\
& e_{2}=w+w \\
& e_{3}=w h \\
& e_{4}=u * c e_{3} \\
& e_{3}=t * c e_{3}+u * e e_{2} \\
& e_{2}=t * e e_{2}+u * e e_{1} \\
& e_{1}=t * \mathrm{ee}_{1}
\end{aligned}
$$

(vi-cola)

(V-Cib)

(V-C1Be)

(VI-C.10d)

(VI-C.10e)

Subroutine EINTG calculates the $\left(d_{j}\right)$ simply as:

$$
d_{j}=\sum_{j=1}^{5} c_{i} e_{j+1-i}
$$

where it is taken that any (e) with subscript outside the range (1-4) is zero.

The integration of Eg. (VI.87) in the general case uses the strategy of factoring $\left(b_{8} / b_{9}+E\right)$ successively from the numerator to create a series of integrals of the form $\int \mathrm{dEf}_{\mathbf{i}} \mathrm{E}^{\mathbf{i}-2}$. Equation (VI.87) is modified:

$$
J\left(m, n b_{g+h}=\frac{1}{b_{9}^{2}} \int_{E_{n}}^{E_{n+1}} d E \sum_{i=1}^{i} \frac{d_{i} E^{j-1}}{(B+E)^{2}} \text {, where } B=\frac{b_{8}}{b_{9}}\right.
$$

EINTG calculates the $f_{i}$ in Eq. (VI.89) as follows:

$$
D_{i}=d_{i}-B\left[d_{i+1}-B\left(d_{i+2}-\cdots B d_{i_{\max }}\right) \cdot \cdot\right]
$$

and from this,

$$
\begin{aligned}
\mathrm{f}_{1} & =\mathrm{D}_{1} \\
\text { and } \mathrm{f}_{\mathrm{i} \neq 1} & =\mathrm{D}_{\mathrm{i}}-\mathrm{B}\left[\mathrm{D}_{\mathrm{i}+1}-\mathrm{B}\left(\mathrm{D}_{\mathrm{i}+2} \cdots \mathrm{BD}_{\left.\mathrm{i}_{\max }\right)}\right) \cdots\right] \text {, }
\end{aligned}
$$




\section{neruances}

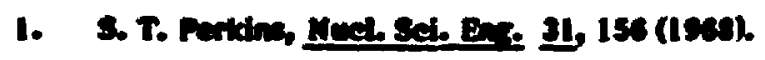

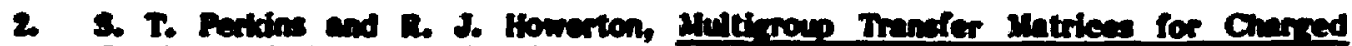

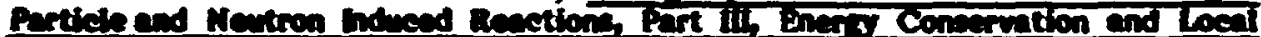

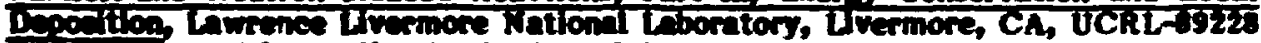
II: I3, sceepted for publication in Nuci. Sef. En.

3. G. L Bell, G. E Hansen, and H. A. Sandmeier, Nuel. Sei. EnT. 28, 376 (1967).

4. S. T. Perkine, Nuel. Sei. En:e 80,130 (1982).

5. S. T. Perkins and P. C. Giles, Nucl. Sei. Eng. 80, 579 (1982).

6. R. J. Doyas and S. T. Perkins, Interpolation Transformations for Two-Dimensional Tabular Data, Lawrence Livermore National Laboratory, Livermore, CA, UChI-51290 (1982).

7. R. J. Doyas and S. T. Perkins, Nuel. Sei. Eng. 50, 390 (1973).

8. R. E. von Holt and R. J. Howerton, Math. Comp. 17, 84 (1963).

9. P. C. Giles, Lawrence Livermore National Laboratory, Livermore, CA, unpublished (1982). 
CHA PIER VI: NDFL

vII $-1 / \gamma_{11}-2$ 


\section{NDPL}

\section{A. INTRODUCTION}

In analyzing complex systems, the simultaneous transport of different particle pecies, Le., neutrons, light charged particles, and gamma rays must be caleulated. In the multigroup sense, these particle fields are coupled through the various particle production matrices. Furthermore, the energy deposition to the thermal ions and electrons and the depletion and accretion of the thermal ion constituents due to muclear reactions luust be taken into account. The same comments apply to the more restrictive fields of reactor neutronies, reactor shielding, and reactor blanket analysis except that charged particle transport is of less importance when dealing with reactor eoncepts.

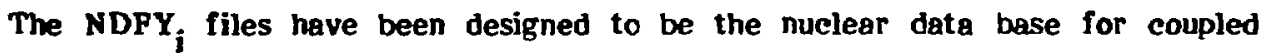
particle multigroup transport unalysis. The suffix $Y_{i}$ refers to the incident particle designator (see Table I-A.1, of Appendix I-A, e.g., NDF2 is the file for which the incident particle is the proton). All information within these files is in binary mode and is generated by the CLYDE code (see Chapter VI.) The function of the NDFL code is multipurpose. It creates the NDFY ${ }_{i}$ files, updates these files, and produces various edits of the files.

In the next section, the organization of the files and their use by the transport codes at problem generation time will be discussed. This will be followed by a description of the input, operation, and output for the various NDFL functions.

\section{B. FILE DESCRIPTION AND USAGE}

Each $N D F Y_{i}$ file contains multigroup data to be used in transport analysis for a specific light particle species. Each target material within a file is designated by its ZA value $\left(1000^{*} \mathrm{Z}+\mathrm{A}\right)$, level excitation energy, and thermal ion temperature. For each material, the following types of data are given: 1. cross sections; 2. types of reaction products for each reaction; 3. rate coefficient for available energy, energy production, and energy to the various outgoing particle fields; 4. particle interaction transfer matrices (higher Legendre moments may be present); and 5. particle production matrices (only $\ell=0$ matrices are present). The layout of the binary $N D F Y_{i}$ file is given in Appendix VIl-A.

For codes that do transport calculations, the $N D F Y_{i}$ files are usually read at problem generation or restart times. The transport code reading the file may collapse the data to fewer groups than are given in the NDFY file. Standard collapse fluxes, group bounds, and atomic weights are coritained in the file, BDFLS, whose layout is given 
in Appentix M-B. BDFLs is also used by various other components of OMEGA, efo, CLYOE. The collepe procedure is described in Appendix VII-C. Group collepse may abo be done by NDFL by using the proper editing option (see Section VII-C.3).

\section{INPUT, OPERATION, AND OUTPUT}

NDFL has the following three functions: (a) to create an NDFY file, (b) to update an $\mathrm{NDFY}_{i}$ file as needed, and (c) to edit an NDFY, file. The procedures used to:

\section{Create un NDPY, file.}

The isput file is a CLYDE output file (in ASCII) witt the transport correction indicator, NTCFLG, set for the CLYDE run $4 \leq \mathrm{NTCF} L G \leq 7$ (see Chapter VD). Several muns of NDFL must be made to create the final file, the first for the particle interaction properties followed by one for each of the particles that can be produced by the varjous interactions. The order in which this is done is: The initial run is for the outgoing particle designator, $y_{o}$, equal to the ineident particle designator, $y_{i}$ (see Table I-A.1). All subsequent runs are for the other possible secondary particles $\left(y_{0}{ }^{\prime} s\right)$, starting with the least value of $\mathrm{y}_{0}$ and going to the greatest.

The ealling sequence for NDFL in this case is:

omega ndfl (input file) (box no.) (hsp option)

(library name) 0 (output file name) (output file size) / $\mathrm{T} V$

The parentheses denote problem dependent quantities and do not appear in the calling sequence. The various quantities in parentheses have the following significance.

input file

box no.

hsp option the file required by NDFL for input, i.e., the CLYDE output.

user's box number

NDFL produces output that is descriptive and/or diagnostic for the problem. The descriptive information is dependent on the type of output called for and is contained in the family of files HSHMMO00, HSHMM001, ..., HSHMM 00X. The diagnostic information contains the input calling sequence and the run description, and is in the file PRT/HMM. The numbers HMM are the three right hand numbers of the machine generated time (24 hour scale) e.g., if the time the problem was generated was 0431 or 1431 , then HMM $=431$. This output may be directed to the FR 80 (use "fr80" for option), the high speed printer (use "hsp" for option), or kept for inspection (use "debug" for option) and/or nutput to RJET or other medium using ALLOUT. 
library name

$\mathbf{0}$

output file name

output file size

the NDFY fike that is being updated. If a new library is being made ab initio, the word "none" is entered here. Sse below.

The zero in this position indicates that the library exists as a file. The zero must be included in the calling sequence.

the name of the NDFY file $_{\mathrm{i}}$ be created as output.

If equal or less than 99 , it is the size of the file to be ereated in units of 100000 . If greater than 99, it is the actual file size to be creater. When the code finishes it will shrink the file to the sicie needed.

\section{Update ar YDFY, File.}

NDFL will delete or insert data for the $\mathrm{NDFY}_{i}$ files on a per material basis only. This is because a change in a single evaluated cross section may change not only the cross sections in the file but also transfer matrices for the outgoing particles and the various energy rate coefficients. Data for a material must be deleted before new data for the material can be inserted so that updating a file requires multiple runs of NDFL.

The input file required to delete the data for a raterial is in the following format:

TABLE VII. l. Input file required to delete the data for a material.

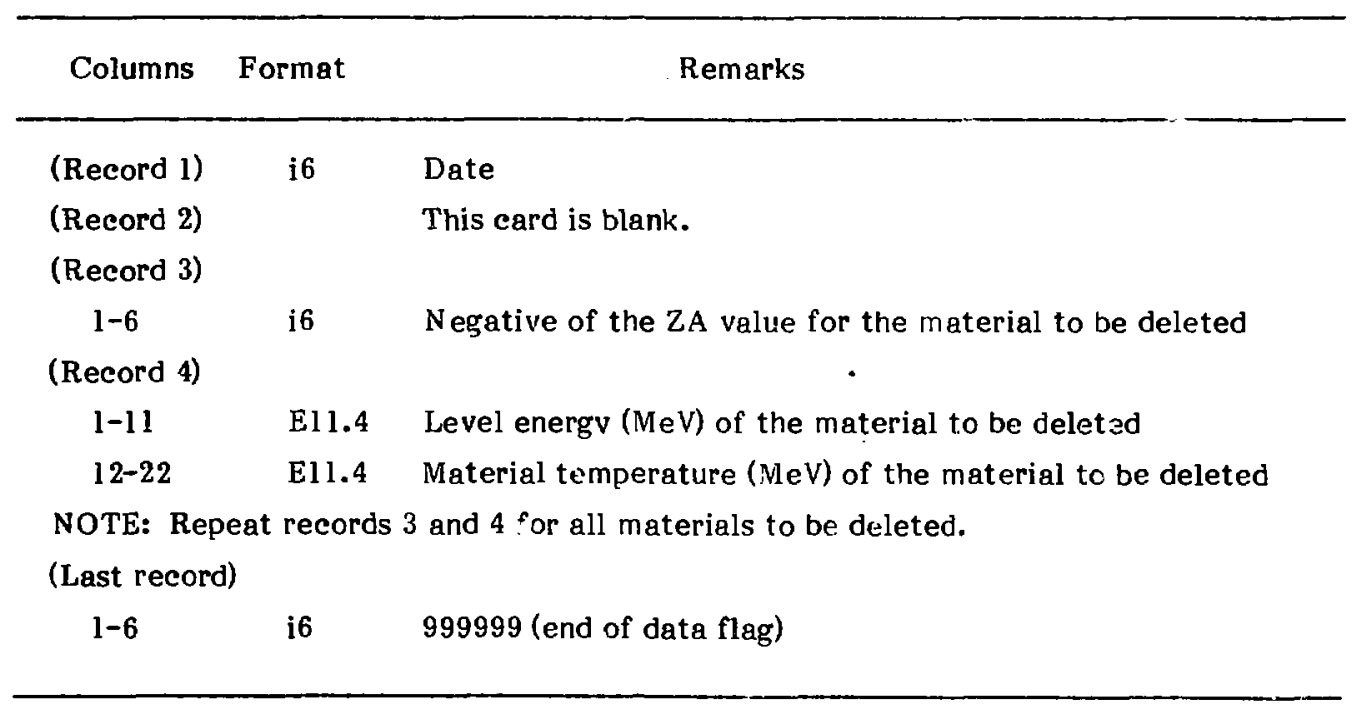

The calling sequence to make deletions with NDFL is identical to that used in creating the file (see Section VII-C.1).

To insert replacement data after $n$ deletion, follow the procedure for creating a file (see Section VII-C.1).

$$
\text { VIl }-5
$$




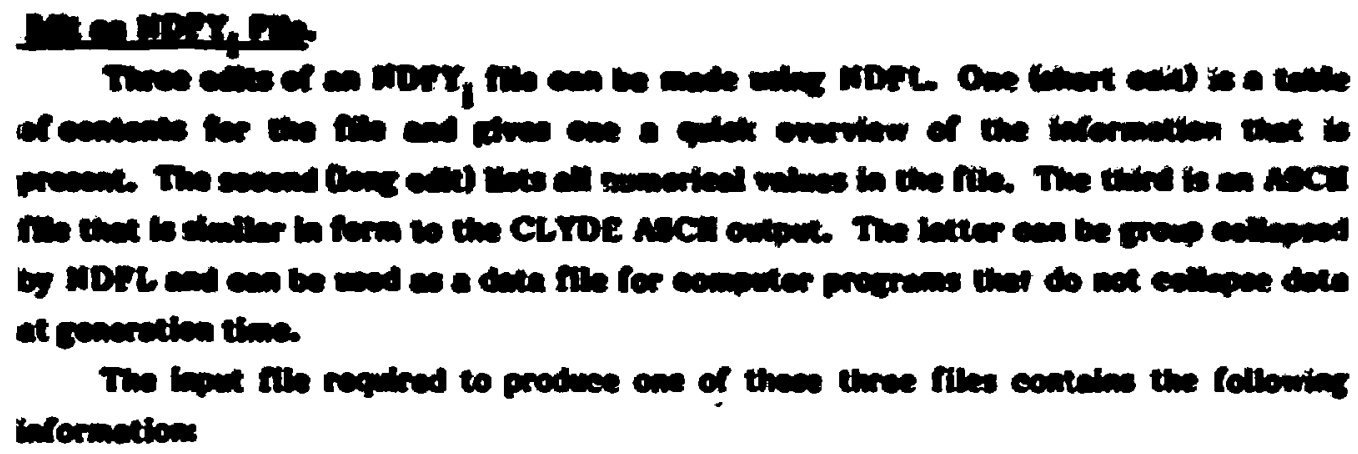

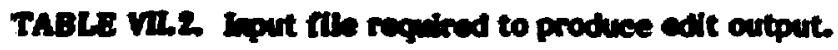

Columans Format Remarks

Reeond 1-1

$3-4$

5-10

11-16 j6

i2

i6

\section{Record Type 1}

\section{NEDTT - specifies type of edit output desired}

1 - reserved for deburg use only

2 - short edit

3 - long edit, similar to CLYDE hsp output

4- ASCII file in the same form as a CLYDE output file

NNZAB - beginning isotope ZA number

N NZAE - ending isotope ZA number

NOTE: NNZAB and NNZAE are specified only when NEDIT $=3$. If NNZAB and NNZAE are both blank, then all isotopes are included in the edit.

NOTE: This is the only input record required for $1 \leq$ NEDIT $\leq 3$.

\section{Record 2-1}

8-9

$11-12$

$14-15$

$17-18$

30

\section{Record Type 2}

IYI - incident particle designator

IYO - outgoing particle designator $(<10)$

NGPI - ineident particle group structure designator from the BDFLS file (see Appendix VII.B). If $\mathrm{NGPI}=0$, then read in incident particle group bounds on Record Type 5.

NFLX - Multigroup weighting flux designator from the BDFLS file (see Appendix VII.B). If NFLX $=0$, then read in multigroup fluxes on Record Type 7.

NPNTOP controls the amount of HSP output and is normally 0. If N PNTOP $=6$, 8 large amount of debug output is given. 


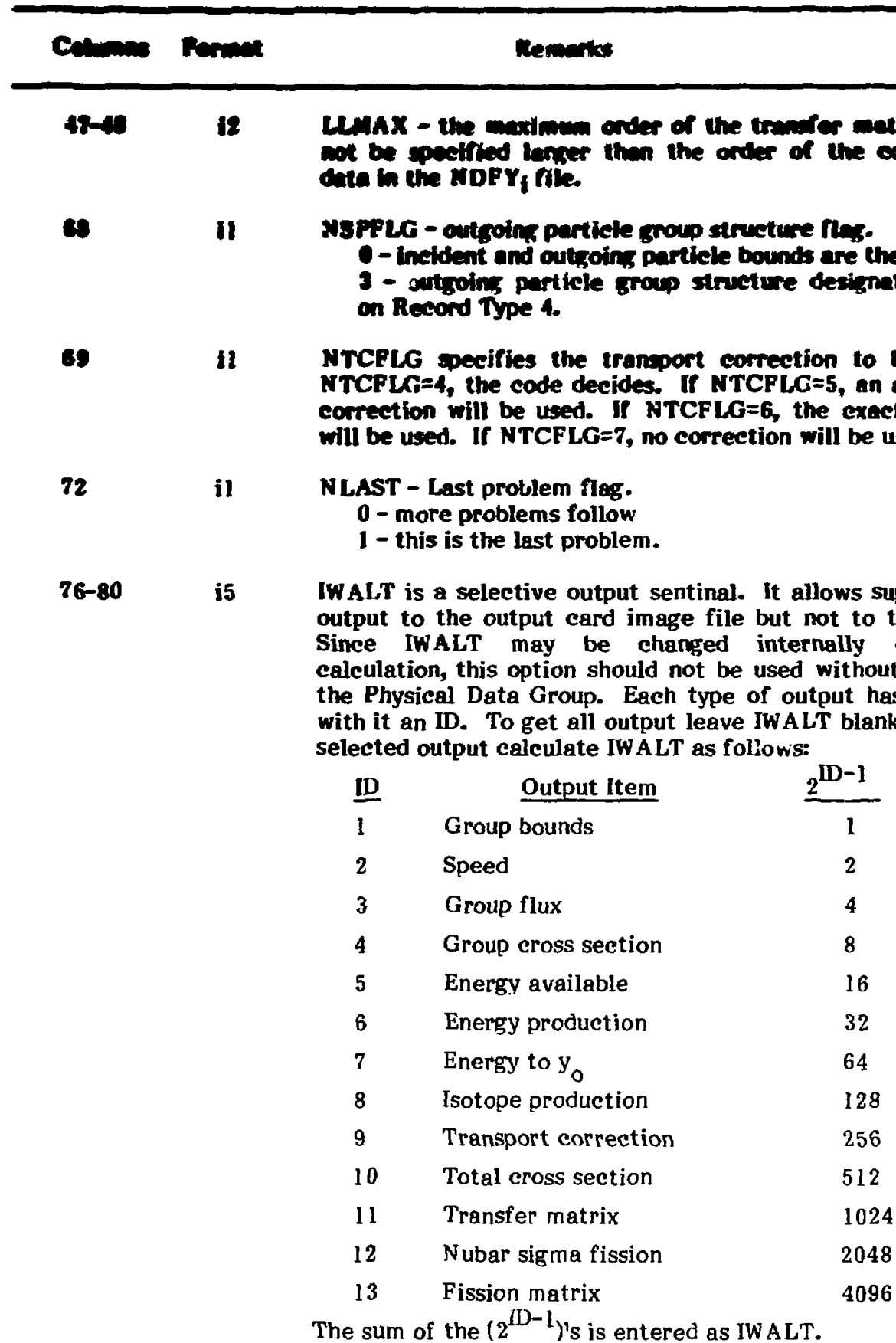




\section{$\frac{2 \operatorname{sen} 15-1}{1-1}$ \\ Lecond $\mathrm{T}, \mathrm{e} 3$ \\ 7-12 \\ 15 \\ MNZAB - Begiming botope zA number \\ Roon 4 \\ $2-3$ \\ i3 \\ Recond Tpe 4 (only specify if NSPFLG $=3$ ) \\ Record 5-1 \\ $1-4$ \\ is \\ NEBI - specifies the number of incident particle group bounds to be found on the following records.}

\section{Record 5-2}

1-11 etc. 6E12.5 EBOUND - group bounds in order of increasing energy (MeV.

Record 5-3, etc.

1-11 etc. 6E12.5 continuation; up through all NEBI values.

NOTE: These bounds must be a subset of the corresponding bounds in the NDFY file.

Record 6-1

Record Type 6 (only read in if NGPO $=0$ )

1-4 i4 NEBO - specifies the number of outgoing particle group

Record 6-2 bounds to be found on the following records.

1-11, ete. 6E12.5 EBOUNDO - group bounds in order of increasing energy (MeV).

Record 6-3, ete.

1-11, etc. 6E12.5 continuation; up through all NEBO values.

NOTE: These bounds must be a subset of the corresponding bounds in the $N D F Y_{i}$ file.

\section{Record 7-1}

$1-2$

Record Type 7 (only read in if NFLX $=0$ )

i2

LFLUX - the highest Legendre order of weighting flux spectrum to be read ( $\geq 0$ and also consistent with LLMAX). 


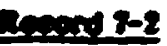

$1-5$

\section{Pand 7-3}

1-11, ete. CE12.5 Energy-flux (in that onder) pairs, for $2=0$ in onder of increasing energy (energy in MeV, flux in Mev-l).

\section{Reend 7-i tete.}

1-11, etc. GE125 continuation up through all NFLUX/2 pairs.

\section{Repeat records 7-3 for all higher onder values of $k$ through LFLUX.}

NOTE: The energies for each $l$ must span the energies of the incident particle group structure. Furthermore, for any required $l^{\text {th }}$ mode weighting flux that is >LFLUX, the values giyen for LFLUX will be used.

The ealling sequence for NDFL in the edit mode is: omega ndfl (input file) (box no.) (hsp option) (library name) 0 / TV

The parentheses denote problem dependent quantities and do not appear in the calling sequence. The various quantities in parentheses have the following significance:

input file

box no.

hsp option the file required by NDFL for input

user's box number

NDFL produces output that is deseriptive and/or diagnostic for the problem. The descriptive information is dependent on the type of output called for and is contained in the family of files HSHMMO00, HSHMM001, .... HSHMM00X. The diagnostic information contains the input calling sequence and the run description, and is in the file PRT/HMM. The numbers HMM are the three right hand numbers of the machine generated time (24 hour scale) e.g., if the time the problem was generated was 0431 or 1431 , then HMM $=431$. This output may be directed to the FR80 (use "fr80" for option), the high speed printer (use "hsp" for option), or kept for inspection (use "debug" for option) and/or output to RJET or other medium using ALLOUT. 


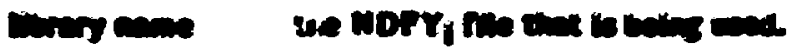

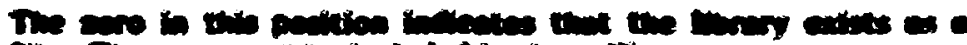

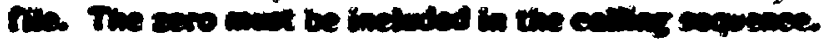

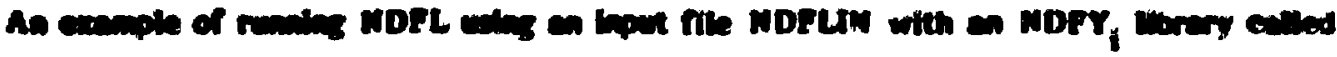 MDI1 mond be}

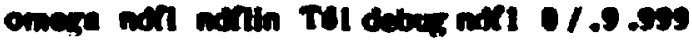

The type of ontput edt depends on the value of the inpot value NEDrT. The st ed't (NDOTrzs eontains the following information:

- ZA mubers of the materials in the library,

- incident particle, maximum $\mathrm{e}^{\text {th }}$ order of interaction transfer matrices, and number of incident groups;

- incident groups bounds, speeds, and group fluxes

- types of cutgoing particles, number of groups, and type of transfer matrix present in the file, e.g, particle conserving;

- first word address and data length of each material; and

- particle types and multiplicities produced from each reaction.

The long edit (NEDIT=3) is similar to the CLYDE hsp output but is larger since it ineludes data for all outgoing particles. It contains all of the information of the short edit ( NEDIT=2) plus the following additional information for each material:

o reaction group cross sections;

- available energy, energy production, and energy transfer to each outgoing particle field;

o group transport correcting cross section up through LMAX+1;

- group total cross section;

- interaction transfer matrices up through LMAX;

- particle production matrices for each outgoing particle field; and

o fission matrix if the material is fissionable.

The ASCII output (NEDIT=4) is contained in the output file NDFOUT. The format of NDFOUT is identical to that of the CLYDE ASCII output (see Chapter VI). The advantage of doing this form of NDFY $_{i}$ edit rather than doing a series of CLYDE calculations is that group collapse, both incident and outgoing particle, can be done directly using multigroup data of the input $\mathrm{NDFY}_{i}$ file. Note that standard fluxes that may be used for group collapse are contained in the ASCII file, BDFLS (see Appendix VII-B).

$$
\text { VII - } 10
$$




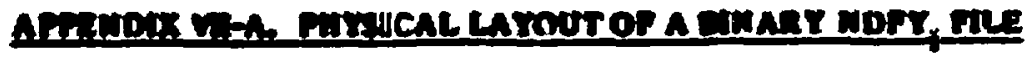

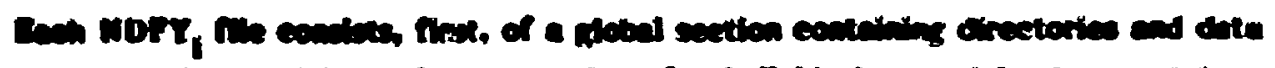

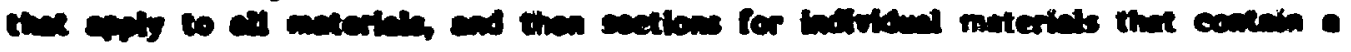

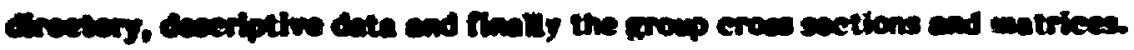

\section{A. GLosal sECTDN}

a. Dincetory - (DRTAB) - Stants at word 1 of NDF Library

1. Incident perticle deniznator

(iyil)

2. Langth of fiobal seetion

(Idir)

3. Date llbrary was made

(Iibdayt)

4. Vaximum size of array needed for individual material directory and definition

(mxdef)

5. Maximum size of array needed for material data

(mxdat)

6. Irput group structure ID

(idgps)

7. Number of groups

(ngps)

8. FW A of group boundary table

(igpfwa)

9. FWA of group speed table

10. Number of collapse weighting fluxes

(ispfwa)

11. Length of coliapse weighting flux table

(newf)

12. FWA of collapse weighting flux table

(lenfl)

13. Number of particle designators in library

(iflfwa)

14. Length of particle designator table

(npp)

15. FWA of particle designator table

(lenpp)

16. Number of target materials in library

(ippfwa)

17. Length of target material designator table

(matnum)

18. FWA of target material designator table

(leniso)

19. Number of supplemental data blocks

(isof wa)

20. Length of supplemental data block array

(nsupp)

(lensup)

21. FWA of supplemental data block array

(isupfwa)

b. Group Boundary Table - (GPTAB) - ngps + 1 values.

(Directory pointers from Aa; 7,8). 


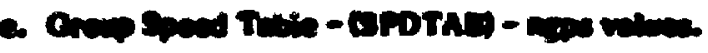

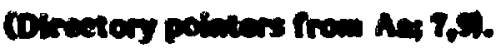

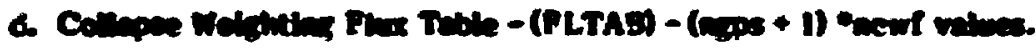

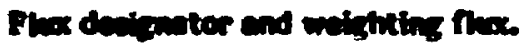

Repeat for all weistulis Puxes.

(Directory pointers from Aas 7, 10, 11, 12.

e. Particle Deaigmator Table - (PPTAB) - 7* npp valuen.

y No, Z, A, corresponding material ZA No, and Eevel if applicable. Abo, nego, the inmber of groups in the particle production matrices, and iecfly, which indicates conservation type.

$0=$ conserves particles

1 = conserves energy production

$2=$ conserves energy

$3=$ conserves particles and energy (group splitting)

Repeat for all particles.

(Directory pointers from Aa; 13, 14, 15).

f. Target Material Designator Table - (ISOTAB) - matnum *5 values.

ZA No., Elevel, KT, FWA and length of individual material directory and descriptor blocks (ISODEF).

Repeat for all materials.

(Directory pointers from Aa; 16, 17, 18).

g. Supplemental Data Blocks - (SUPTAB) - $c$ (length of each data block) values. Designator and length of data block followed by data.

Repeat for all designators.

(Directory pointers from $A$ a; 19, 20, 21).

B. INDIVIDUAL MATERIALS - DIRECTORY AND DESCRIPTOR BLOCKS (ISODEF)

a. Directory

Locations within ISODEF array with respect to first location in the array for each material. The Directory, $\mathrm{Ba}$, is 50 locations long. The Reaction Descriptor Block, Bb, starts in location 51 . 


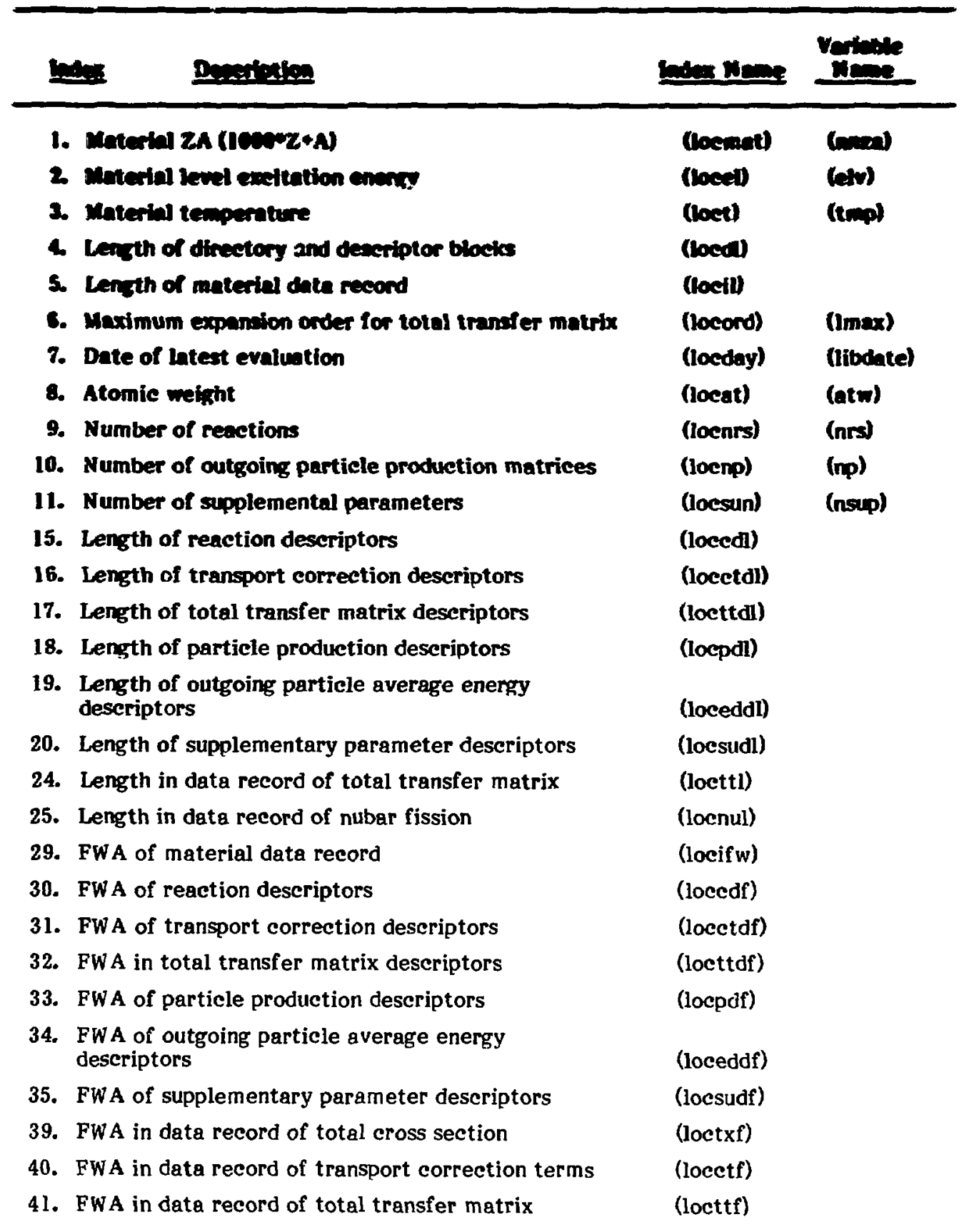




\begin{tabular}{|c|c|}
\hline 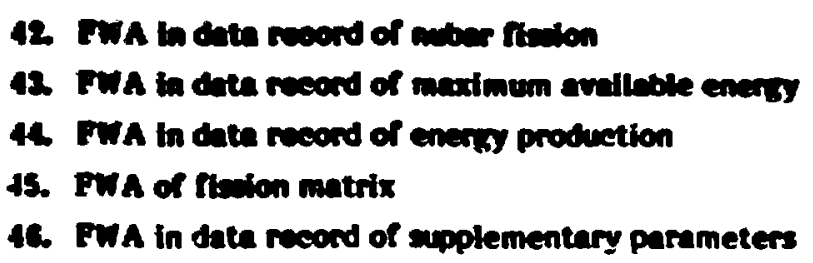 & $\begin{array}{l}\text { (bemi) } \\
\text { (boemx) } \\
\text { (loceph) } \\
\text { (loefis) } \\
\text { (loceun }\end{array}$ \\
\hline
\end{tabular}

b. Reaction Descriptor Block

$9 *$ nrs $+2 *$ nrs npp (nrs) values $C, S, Q, X 1, X 2, \times 3, Q_{\text {eff }}$ and FWA of the reaction $\mathrm{C} / \mathrm{s}$ data. This is followed by the number of pairs, (npp(c)), of (ZA, multiplicity) being produced from this reaction and the corresponding values of these pairs. By definition, a multiplicity of zero designates a value that is group dependent, e.g., $v_{g}$ for neutrons. Repeat for all reactions.

Data pointers from Ba; $9,15,30$.

c. Outgoing Perticle Average Energy Descriptor Block

$2 *$ np values

$Y_{0}$, and FWA of outgoing particle average energy data. Repeat for all values of $\mathbf{y}_{\mathbf{o}}$

Data pointers from Ba; 10, 19, 34 .

d. Transport Correction Descriptor Block

$2 *(1 \max +1)$ values

Value of $\ell$ and FWA of transport correcting term data. Repeat for all values of $\ell$ through $\operatorname{lmax}+1$.

Data pointers from $\mathrm{Ba} ; 6,16,31$. 


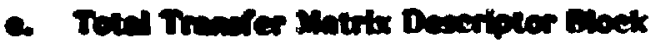

$(S+2 *$ mpal $*(\operatorname{limax}+1)$ wives

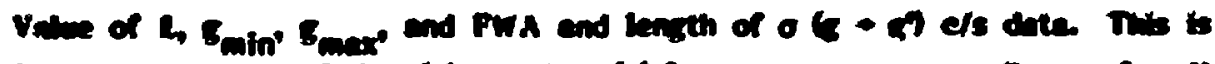

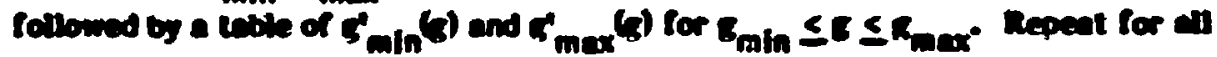
velues of 2 throunth inax.

Date pointers from Ban 4, 17, 32.

P. Outpoing Particle Production Descriptor Block

$(6+2 *$ ngpel * np values

$y_{O g}$ idogs, $8_{\min }, 8_{\max }$, and FWA and length of $\sigma(g+8) \mathrm{c} / \mathrm{s}$ date. This is followed by a table of $g_{\min }(\mathrm{g})$ and $\mathrm{g}_{\max }(\mathrm{g})$ for $\mathrm{g}_{\min } \leq \mathrm{g} \leq \mathrm{g}_{\max }$. Repeat for all values of $y_{0^{*}}$. ldogs is in the output group structure ID.

Data pointers from Ba; 10,18,33.

g. Supplemental Parameters Descriptor Block

Data pointers from Ba; 20, 35.

C. INDIVIDUAL MATERIALS DATA BLOCK

a. Total cross section

ngps values

Data pointers from Ba; 39

b. Reaction cross sections

$\sum_{\text {nrs }} g_{\max }(C)$ values

Data pointers from $\mathrm{Bb}$

c. Transport correcting cross sections

$(1 \max +1) *$ ngps values

Data pointers from $B a ; 40$, Bd

d. Total transfer matrix $\left(y_{i} \rightarrow y_{i}\right)$

$$
(1 \max +1)^{*} \sum_{g_{\min }}^{g_{\max }}\left(g^{\prime}{ }_{\max }(g)-g^{\prime} \min (g)+1\right) \text { values }
$$

Data pointers from $\mathrm{Ba} ; 24,41$; $\mathrm{Be}$ 
- Ompins particle production maturi $\left(y_{1} \bullet y_{0}\right)$

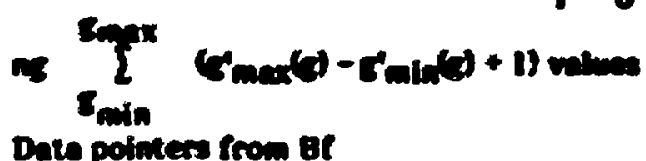

f. Supplemental parametcis data

$\sum_{\text {nip }}$ (size of each data)

Date pointers from Ba; 11, 46; Bg

6. Sigma*mbar fision data

nzps values

Data pointers from Ea; 25, 42

h. Maximum available energy data

ngps values

Data pointers from $\mathrm{Ba} ; \mathbf{4 3}$

i. Energy production data

ngps values

Data pointers from Ba; 44

j. Outgoing particle average energy data

ngps*np values

Data pointers from Bc

k. Fission matrix

ngps $* * 2$ values

Data pointers from $\mathrm{Ba} ; 45$ 


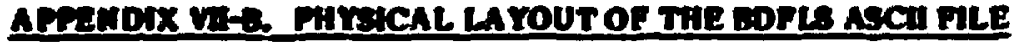

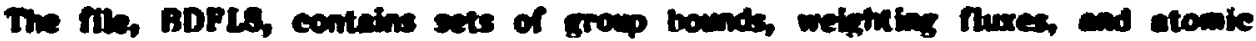

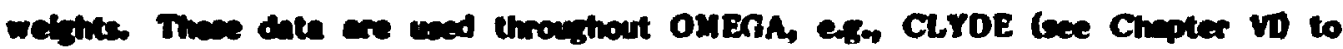
produce nuitizroup constants, and by programs other than OMEGA, esen to collopse the croses sections at generation time.

BDFLS containg group bounderies correspondinas to each incident particle ( $\left.y_{\mathbf{z}}\right)$. For each incident particle, there is one master set, i.e., the set with which the correaponding NDFY, file was generated. There are also several additional group structures for the same incident particle. Each of the additional sets is a subset of the master set and is used for group collapse. The weighting fluxes are given as point wise data, linear-linear interpolable in energy. These fluxes are used both for group collapse and to generate the multigroup data with CLYDE.

The format of the BDFLS file is as follows:

TABLE VII-B.1. BDF LS file format.

Columns Format Remarks

Record 1-1

1-4 i4 Group bounds ID number.

5-44 5A 8 Alphanumeric information.

Record 1-2

1-4 i4 Number of group bounds.

Record 1-3

1-11,etc. 6E12.5 Group bounds in order of increasing energy (MeV).

Record 1-4

1-11,etc. 6E12.5 continuation of record 1-3; up through all group bounds.

NOTE: Continue through all sets of group bounds in the file. Follow the last set with a card which is blank except for a 1 in column 73.

$\underline{\text { Record 2-1 }}$

GROUP FLUX CARDS - Record Type 2

$\begin{array}{lll}\text { 1-4 } & \text { i4 } & \text { Group flux ID number. } \\ \text { 5-44 } & \text { 5A } 8 & \text { Alphanumeric information. }\end{array}$

VII -17 


\section{Reeond 2-2}

$1-4$ is

LAAX, the highest 2 order for the Legendre moments of the weighting flux. LMAX+1 subsets of weighting fluxes are given (LMAX $\geq 0$ ).

\section{Reeard 2-3}

$1-4$

i4

Reeord 2-4

$1-4$

i4

Repeat record 2-3 foi all l's up through LMAX.

Record 2-5

1-11,etc. 6E12.5 Energy flux (in that order) pairs, for $t=0$ in order of increasing energy (energy in MeV, flux in $\mathrm{MeV}^{-1}$ ).

Record 2-6

1-11,etc. 6E12.5 Continuation of record 2-5 up through all entries for $\ell=0$.

\section{Record 2-7}

1-11,ete. 6E12.5 Repeat records 2-5 and 2-6 for all $\ell$ 's up through LMAX.

NOTE: Continue through all sets of group weighting fluxes in the file. Follow the last set with a card which is blank except for a 1 in column 73.

Record 3-1

$$
\text { ATOMIC WEIGHT CARDS - Record Type } 3
$$

$$
\begin{array}{lll}
5-10 & \text { i6 } & \text { ZA number, i.e., } 1000 \mathrm{Z}+\mathrm{A} . \\
11-22 & \text { E12.5 } & \text { Atomic weight (amu). }
\end{array}
$$

NOTE: Continue through all $\mathrm{ZA}$-atomic weight pairs in the file. Follow the last set with a card which is blank except for a $l$ in column 73 .

5-10 i6 999999.

FILE END CARD 


\section{APRENDIX W-C GROUP COLLAPSE OF THE NDPY, PLLES}

Conider an MDPY, nie for a given incident particle whose group structure is desizmated a f. Within the ille are group crose sections, partiele interaction tranafer matrices, ete., which are based on this grow structure. Also in the file are particle production matrices with group structures in general not the same as each other and not the same as the incident particle group structure. Designate one of the outgoing particle group structures as $\mathrm{f}$. To collapse these group constants to a subset of the original, define the following group structures:

incident farti- le groups

$$
\begin{array}{ll}
\mathrm{f} \text { (uncollapsed) } & \mathrm{I} \leq \mathrm{j} \leq \mathrm{J}, \\
\mathrm{g} \text { (collapsed) } & 1 \leq \mathrm{g} \leq \mathrm{G} ;
\end{array}
$$

final particle groups

$$
\begin{array}{ll}
\mathrm{j}^{\prime} \text { (uncollepsed) } & 1 \leq \mathrm{j}^{\prime} \leq \mathrm{J}{ }^{\prime}, \\
\mathrm{g}^{\prime} \text { (collapsed) } & 1 \leq \mathrm{g}^{\prime} \leq \mathrm{G}^{\prime} .
\end{array}
$$

Group 1 in any of the group structures is that of highest energy. To determine the limits for the summations, define for a given group $\mathrm{g}$,

$$
\begin{aligned}
& \mathbf{j}_{\min }=\text { minimum } \mathbf{j} \text { in } \mathrm{g}, \\
& \mathrm{j}_{\max }=\operatorname{maximum} \mathbf{j} \text { in } \mathrm{g} .
\end{aligned}
$$

and for a given group $\mathrm{g}$ ',

$$
\begin{aligned}
j_{\min }^{\prime} & =\operatorname{minimum} j^{\prime} \text { in } g^{\prime} \text { for } g^{\prime} \neq l \\
& =I \text { for } g^{\prime}=1 \\
j_{\max }^{\prime} & =\operatorname{maximum} j^{\prime} \text { in } g^{\prime} \text { for } g^{\prime} \neq G^{\prime} \\
& =J^{\prime} \text { for } g^{\prime}=g^{\prime} .
\end{aligned}
$$

These latter bounds on $\mathbf{j}$ ' insure particle conservation. Note further that the group boundaries for the group structure $g$ are a subset of the boundaries for the group structure $j$. This also applies for the group structures $g^{\prime}$ and $j^{\prime}$. In the NDFY files, transfer matrices are iriven up through order $\ell_{\max }$. Transport correcting cross sections are given up through $/_{\text {max }}+1$. At transport calculation generation time, particle interaction transfer matrices are required up through order $L$ and transport correcting cross sections only for order $L+1$, where $L \leq \ell_{\text {max }}$.

$$
\text { VII }-19
$$




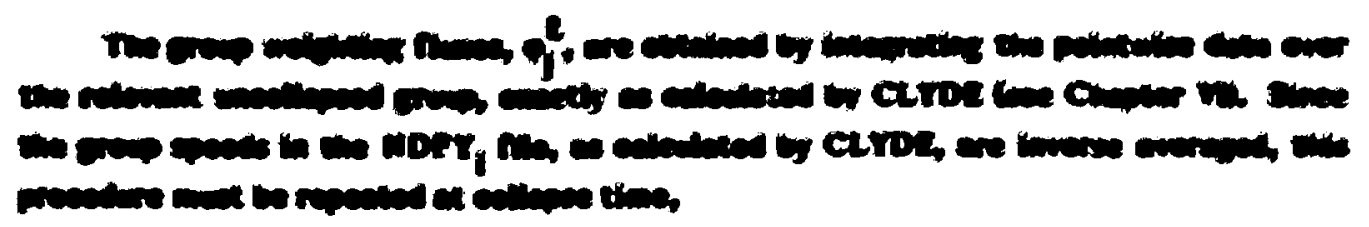

$$
\nabla=\frac{29}{2 \frac{9}{5}}
$$

The grop erous scetions are eolleped by the relation

$$
\sigma_{E}=\frac{\sum \sigma_{j} q_{j}^{0}}{\sum \nabla_{j}^{0}}
$$

With one exception, the energy rate coefficients, ise, available energy, energy production, and energy to particle field $y_{0}$, are collapsed analagous to the group cross seetions, Eg. (VII-C.6). This exception occurs when group splitting is used in CLYDE, i.e., the parameter IECFLG=3 (see Chapter VI), and will be discussed below.

Transfer matrix collapse depends on which IECFLG option was used by CLYDE to generate the data. This will be diseussed below.

IECFLG $=0$ (conserves particles)

For all $\ell$, one has

$\sigma_{\ell+g^{\prime}}=\frac{\sum \sum_{j}^{\sum} \sigma_{l_{j+j}} \varphi_{j}^{l}}{\sum_{j} \varphi_{j}^{l}}$.

IECFLG $=1$ (conserves energy production)

In this case,

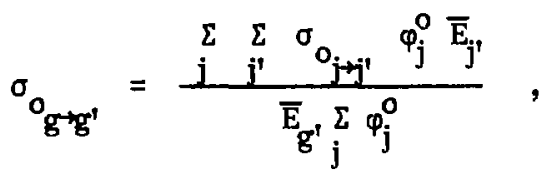




$$
\begin{aligned}
& \left.e_{p}=\left(c_{p+1}+2\right)\right)^{2} \\
& 8=100+1+2) 12
\end{aligned}
$$

(wires)

(vinic)

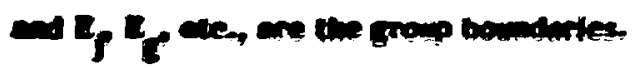

IECPLE $=2$ (comers energy)

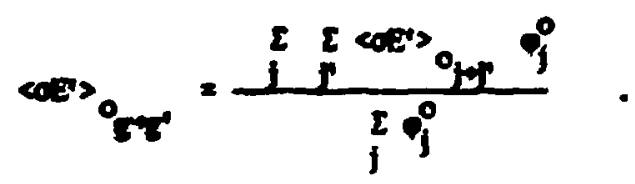

(vir-c.II)

IECFLG = 3 (conserves particles and energy, ide., group splitting)

Group splitting is done only for the $l=0$ transfer matrices. For all $\ell$, calculate $\sigma_{\ell_{g}}{ }$ from Eq (VII-C.n). Since the average energy of the outgoing particles in group $\vec{j}$ is equal to $E_{j}$ [see Eg. (VI-C.g) and Chapter VD, the average energy of the outgoing particles in group $\mathrm{g}^{\prime \prime}$ (for a given group $\mathrm{g}$ also) is,

$$
\langle E\rangle_{g^{\prime}}=\frac{\sum \varphi_{j}^{0} \sum \sigma_{j_{j j^{\prime}}} \bar{E}_{j^{\prime}}}{\sigma_{\sigma_{g^{\prime} g^{\prime}} \sum_{j} \varphi_{j}^{0}}} .
$$

Define $\tilde{\sigma}_{o_{g-g^{\prime}}}$ as the $\ell=0$ transfer matrix after group splitting. Then, proceeding exactly as with the method used in CLYDE (see Chapter VI) with the definition of the

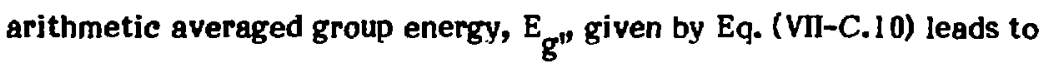

$$
\begin{aligned}
& \text { for }\langle E\rangle_{g^{\prime}}\left\langle E_{g^{\prime}}\right. \text { (splitting to lower energy): } \\
& f_{g^{\prime}}=\frac{\langle E\rangle_{g^{\prime}}-\bar{E}_{g^{\prime}+1}}{\bar{E}_{g^{\prime}}-\bar{E}_{g^{\prime+1}}}, \\
& \tilde{\sigma}_{O_{g \rightarrow g^{\prime}}}=f_{g^{\prime}} \sigma_{{ }_{g} g^{\prime}},
\end{aligned}
$$

$$
\text { VII }-21
$$




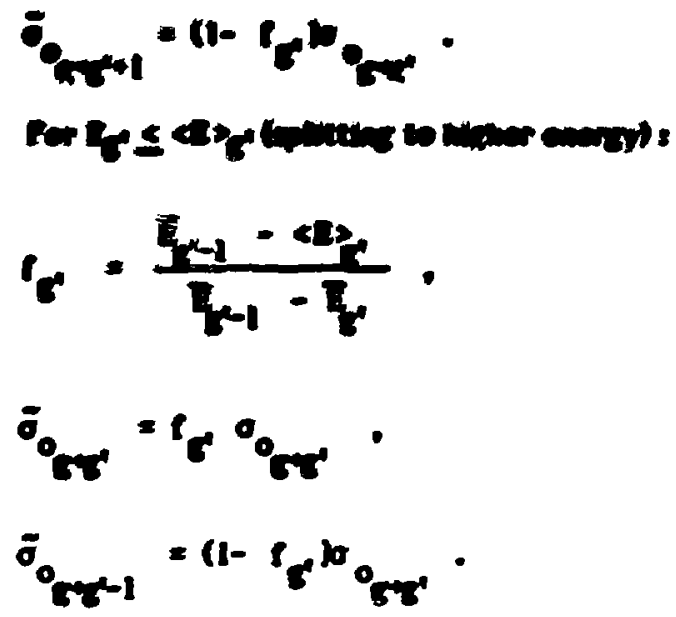

(civerats)

(veria

(vitcin)

Note that the croup split matrix element, $\tilde{\sigma}_{0}$

may receive contributions from snoups $\mathrm{g}^{\prime}-1, \mathrm{~g}$, and $\mathrm{g}^{+1}$. There are two cases in which no splitting is done, i.e,

$$
\tilde{\sigma}_{\mathrm{g} \mathrm{Bg}^{\prime}}=\sigma_{\mathrm{O}_{\mathrm{B}} \mathrm{g}^{\prime}} .
$$

These oceur when

$$
\begin{aligned}
& \mathbf{g}^{\prime}=\mathrm{G}^{\prime} \quad \text { with }\langle E\rangle_{\mathbf{g}^{\prime}}<\mathrm{E}_{\mathbf{g}^{\prime}}, \\
& \mathbf{g}^{\prime}=1 \quad \text { with } \mathrm{E}_{\mathbf{g}^{\prime}}<\langle E\rangle_{\mathbf{G}^{\prime}},
\end{aligned}
$$

Thus particles cannot be split out of the collapsed group structure. Group splitting to a final group structure, when done directly by CLYDE, does not, in general, result in the same final transfer matrix as when done by the group collapse method. Both particles and energy however, are conserved in both cases. As was noted above, the energy rate coefficient for deposition to particle field $y_{o}$ is different under group splitting than when group splitting is not done. For group splitting and including the two special cases deseribed above,

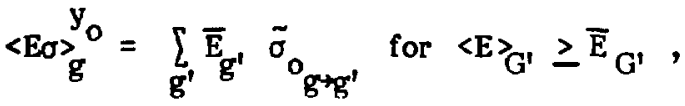

$$
\begin{aligned}
& =\sum_{g^{\prime}} \bar{E}_{g^{\prime}} \tilde{\sigma}_{\sigma_{g} g^{\prime}}+\left[\bar{E}_{G^{\prime}} \sigma_{o_{g \rightarrow G^{\prime}}}-\left\langle\sigma E^{\prime}\right\rangle_{\sigma_{g \rightarrow G^{\prime}}}\right] \text { for }\langle E\rangle_{G^{\prime}}\left\langle\bar{E}_{G^{\prime}} \cdot\right.
\end{aligned}
$$

$$
\text { VII }-22
$$




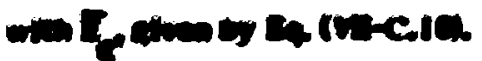

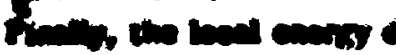

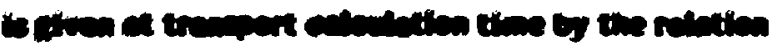

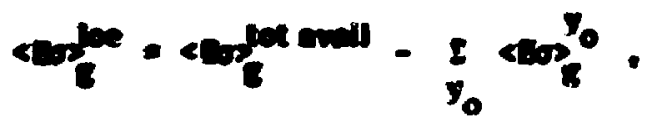

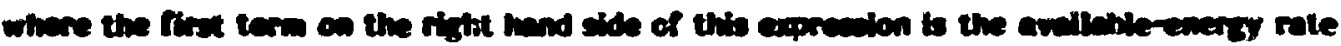

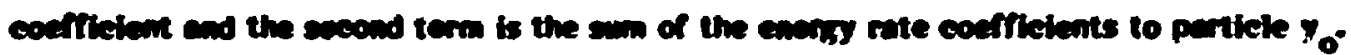
The sumination over $y_{0}$ ineludes onis thow partieles that will be folbowed in the trenport ealeniations.

CLYDE can ealeulate the transport correcting eross section by three different methods (see Section C.2 of Chepter VD. These are designated as "exset," "epproximate," or "the code chooses" The same technique will be used for the NDFL edit outpurt option. The files will be made utilizing the approximate form of the transport correcting eross sections, which from Eq. (VL.28) is

$$
\sigma_{\text {tr } \operatorname{cort}_{j}}(l)=\sum_{j^{j}} \sigma_{\ell_{j+j}}
$$

for $1 \leq \ell \leq \ell_{\max }+1$.

Then on edit request, NDFL calculates analagous to CLYDE results, for $\ell \leq L+1$,

1. exact

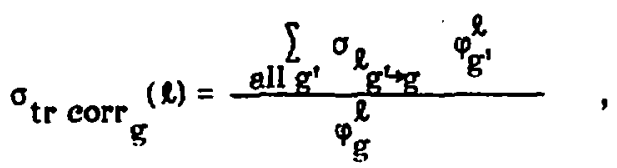

and 2. approximate

$$
\sigma_{\text {tr } \operatorname{corr}_{g}}(l)=\frac{\sum_{j} \sigma_{\operatorname{tr} \operatorname{corr}_{j} \varphi_{j}^{\ell}}^{\varphi_{j}}}{\sum_{j}^{l}},
$$

this latter form using directly the uncollapsed results. It should also be noted that if the uncollapsed transport correcting cross section had been generated by CLYDE in the 


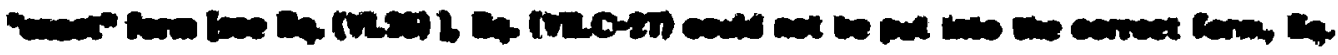

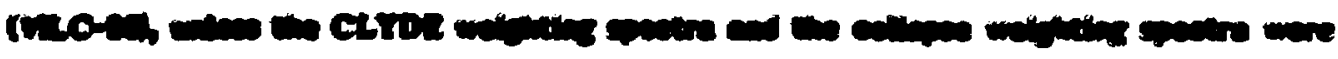

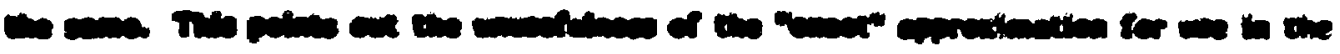

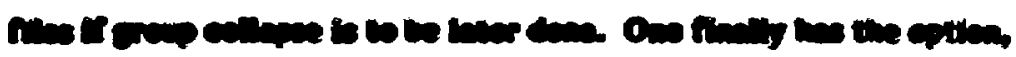

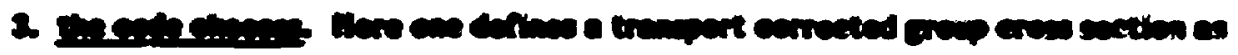

$$
\sigma_{f}(t)=\sigma_{t}-\sigma_{\text {to }} \cos _{c}^{(t)}
$$

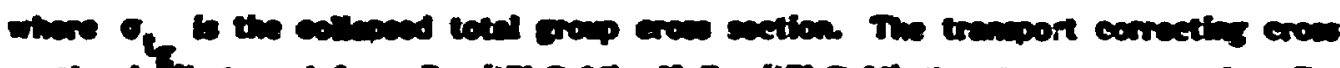

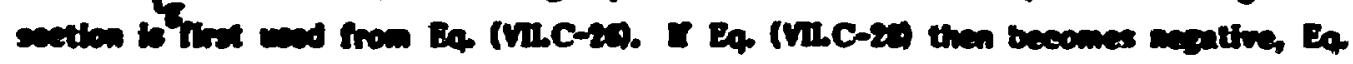

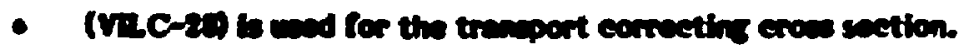

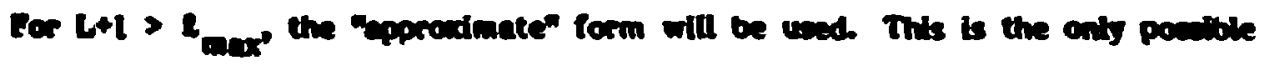
option hase slmee the tremefer matrix for $\ell_{\max }+1$ bs not in the files. 
CHAPTER VII: TART

$$
\text { VIII - } 1 / V i i l-2
$$




\section{v. craner}

\section{A. INThoouction}

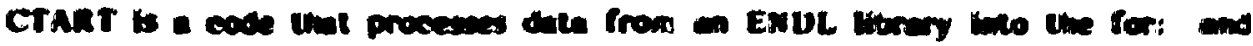

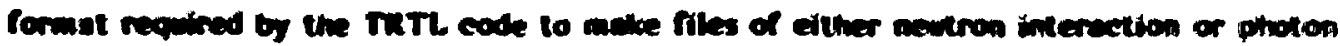
production date required by the TARTN P and other LXXL codes that we the Nome Carto peckane developed sor the TARTX P code. The calling sequence for ruming CTAKT ive

\section{OVEGA CTART (input fike) (box mo) (hap cption) (library rama) \\ o (output file namel (output file size) / T V.}

The parentheses denote problem depentent quantities and do not appenr in the calling sequence. The various quantities in parentheses have the following significance:

input file

box no.

hsp option

library name

0

output file name

output file size the file required by CTART for input

user's box number

CTART produces output that is deseriptive and/or diagnostic for the problem. This output may be directed to the FR80 (use "fr80" for option), the high speed printer (use "hsp" for option), or kept for inspection (use "debug" for option) and/or output to RJET or other medium using ALLOU'T.

the library that is being used for the calculation.

The zero in this position indicates that the library exists as a file. The zero must be included in the calling sequence.

the name of the file to be created as a data file for use by the TRT'L code to make a TARTND or TARTPPD data file.

the size of the output file to be created in units of 100000 words. In the example below, the output file will be created as 2000000 words. When the code is finished it will shrink the file to the size needed.

An example of running CTART using an input file CTIN with a library called ND821201 to make a data file CTOUT that can be used with the TARTNP code is: omega etart etin t64 fr80 nd8212010 ctout $20 / .9 .999$ 
1. mipanaw or anot

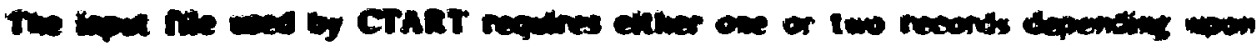

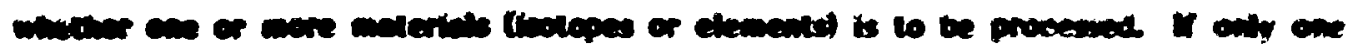

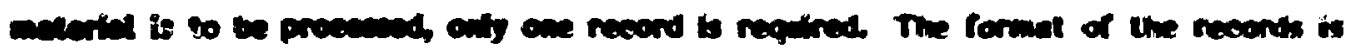

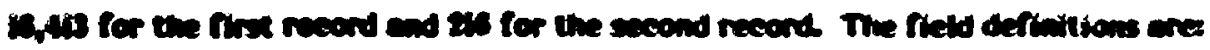

Cociman Value Simbificance

\section{Plet Reood}

1-6 2A

0

7-9 Yi

10-12 Yo

13-15

6

$16-18$
ZA value of element or isotope if only one is to be processed.

Flag that indicates a range of $\mathrm{ZA}$ values is to be processed. The range will be given on the second record.

The numerical equivalent of the incident particle. See Table IA-1 of Appendix IA.

The numerical equivalent of the secondary particle type for which this run is being made. See Table $\mid \dot{i}-1$ of Appendix IA.

Number associated with the energy mesh for which mean value cross sections etc. are to be calculated. For the standard TARTN P data file the entry is 6. For other meshes special arrangements must be made. See R.J. Howerton or R.E. Dye.

Signifies that a flat flux is to be used in averaging the quantities for which mean values will be calculated.

Second Record (if needed)

1-6 ZAl

Least ZA value to be processed.

7-12 ZA2

Greatest $\mathrm{ZA}$ value to be processed. 


\section{c tolmors}

10 coners

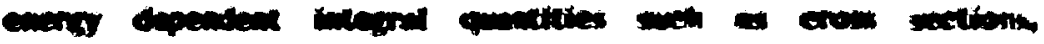

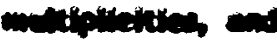

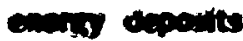
the the the the the

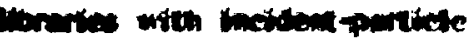
1 1

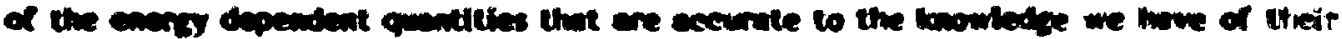

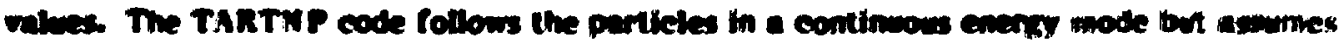

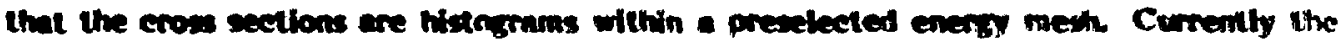

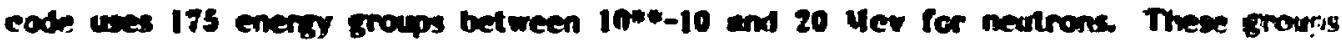
were selected to be more dense in important enesy regimes and less dense in less Importent ener,y rejimes. The importance of an energy regime has been determined, for example, by determining whether important reactions have their thresholds in that range of energies. The histogram values are obtained in CTART by calculating the integral mean value of the quantity in question within the boundaries of each group. Thus, in the usual parlance of group neutronies or photonies theory, the cross sections are flat-weighted group cross sections. For those reactions that threshold within a group, the group cross section is for the entire group so that it has a norzero value below the threshold. This is clearly an unacceptable situation that is corrected by the TARTNP code by checking for the threshold and rejecting the reaction type if the particle energy is less than the threshold energy.

In the TARTNP code total and local energy deposits are used for each incident particle energy group for each isotope or element. The total eriergy deposit is the cross section weighted sum of the average secondary particle energies and residual nucleus energy for all particles except the particle being followed in the problem. The local energy deposit is a similar cross section weighted sum but the average gamma-ray energy is excluded. CTART first calculates the sum of the average secondary energies for all particles other than the particle being followed and adds to that the average energy of the residual nucleus, if any, for each reaction to obtain the total energy deposit for the reaction. A similar sum is calculated excluding the average photon energy and this is the local energy deposit for the reaction. These two sums are then cross section weighted and averaged over the energy groups to form the group total and local energy deposits for the isotope or element.

Angular and energy distributions of secordary particles of the type being followed in the TARTNP code are transformed from their differential form, as they are represented in the libraries, to an integral form for the TARTNP code. More explicitly, each angular 


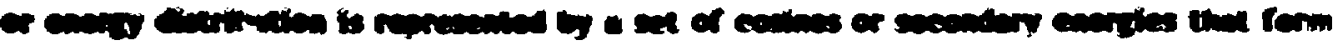

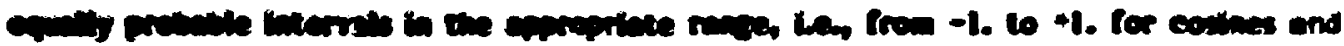

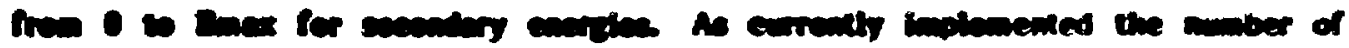

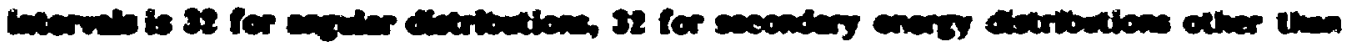

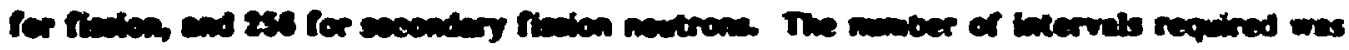

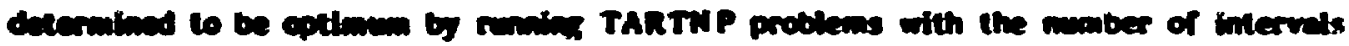
vaind to tont the resits.

\section{OUTPut}

The oupet from the CTART code is a tile that can be uned by the TRTL code to elther create or update a data file that can be used by the TARTNP code. In addition to the file that will be used by the TRTL code, there are two other output files that are ereated by CTART. One describes the input and output of the problem and the other gives any dingnosties that the code might write. Depending upon the option used for the "hop cption" in the calling sequence of the code, these iwo files will either be sent for printing or will be kept in the case of using the "debug" option. If the latter is used the first of the two files will be labeled in the form "HSTTTOOO" and the lattei "PRT/TTT". The "TTT" in each file is the hour and minute that the problem was run with the convention that the hour is designated by one digit so that at 10:00 AM the number in the first place is 0 etc. If there is more output than can be contained in a single file the "HSTTT000" file will be closed and a new file "HSTTT001" will be opened. This procedure of creating new files will continue until no more are needed. The amount of output that is generated for the "PRT/TTT" file is relatively small so that only one file is needed.

The layout of the data files produced by CTART is given in Appendix VIll-A. 
CHA PTER IX:

TRTI.

$$
\mathrm{IX}-1 / \int^{2} x-2
$$




\title{
1x. TIR
}

\section{A. IATHoovcton}

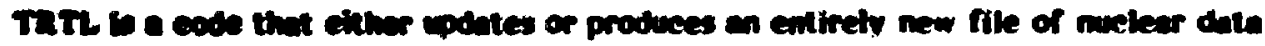
in the form ropined by the TARTNP eode and other LLNL eodes that use the Honte Curlo packose developed for TARTN P. The celling sequence for ruming TRTL is:

\section{OMEGA TRTL (input file) (box na.) (hap option) (olft file name) \\ O (output file name) (outout file size) / T V.}

The parentheses denote problem dependent quantities and do not appear in the calling sequence. The various quantities in parentheses have the following significance:

\begin{abstract}
input the file required by TRTL for input. This file will be an output file from the CTART code.

box no.

user's box number

hsp option

TRTL produces output that is descriptive and/or diagnostic for the problem. This output may be directed to the FR80 (use "fr80" for option), the high speed printer (use "hsp" for option), or kept for inspection (use "debug" for option) and/or output to RJET or other medium using ALLOUT.

old file name the name of the existing data file that is to be updated. If an entirely new file is to be made a file called DT041773 must be used. The latter may be obtained by using XPORT with the command rd .415600:dvt:dt041773.

0 The zero in this position indicates that the library exists as a file. The zero must be included in the calling sequence.

output file name the name of the file to be created as a data file for use by the TARTN P code.

output file size the size of the output file to be created in units of 100000 words. In the example below, the output file will be created as 2000000 words. When the code is finisher it will shrink the file to the size needed.
\end{abstract}

An example of running TRTL using an input file CTOUT with a data file called tl821201 to make a data file called t1821202 is:

omegs trtl ctout t64 fr80 tl8212010 t1821202 20/.9.999 


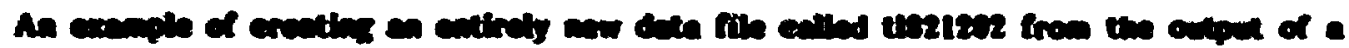
Crant mened crantour b

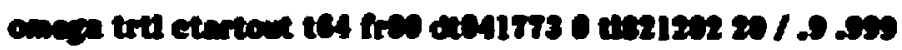

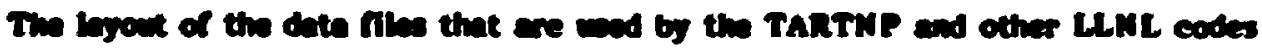

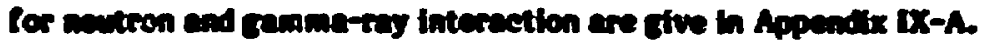




\section{APFENDIX 1X-A}

\section{Disk lygent of metron and gamma ray production date files.}

\section{Pirst}

Contents

Addres

0

1

2

$2+\mathbf{N} \mathbf{M}$

$2+2 * N M$

$2+3 * \mathrm{NM}$

$\mathbf{N} \mathbf{M}$

$2+4 * N M$

JMAX EGV(J)

2+4*NM+JMA.X JMAX EGM(d)

III $=2+4 * \mathrm{~N} M+2 * \mathrm{JMAX}$

III

IN DX

III +INDX INDX

III +2*INDX INDX

II $1+3 *$ INDX $\quad$ INDX

III $+4 *$ iNDX $\quad$ INDX

II $2=F W A(1)-5$

\section{$\mathbf{0}$}

NM Number of Materials (1sotopes or elements) in the file if $N M$ is greater than 100. If the number of materials is $\leq$ $100, N M=100$ (see Note 1 ).

$\mathrm{ZA}(\mathrm{M})$ where $\mathrm{ZA}(\mathrm{m})$ is $1000^{*} \mathrm{Z}+\mathrm{A}$ for material $M$, e.g., for $M=7$ the material is ${ }^{6} \mathrm{Li}$ so $\mathrm{ZA}(7)=3006$, for $\mathrm{M}=18$ the material is elemental $\mathrm{Mg}$ so $\mathrm{ZA}(18)=12000$.

NM FWA(M) The first word address of tise data in the file for the material (M).

L(M) The number of words in the data file for the materia! (M).

AW(M) Atomic weight of material (M) in amu.

Energy group limit in speed units $(\mathrm{cm} / \mathrm{sh})$ for group limit J. Energy group limits in energy units (MeV) for group limit $\mathrm{J}$. 


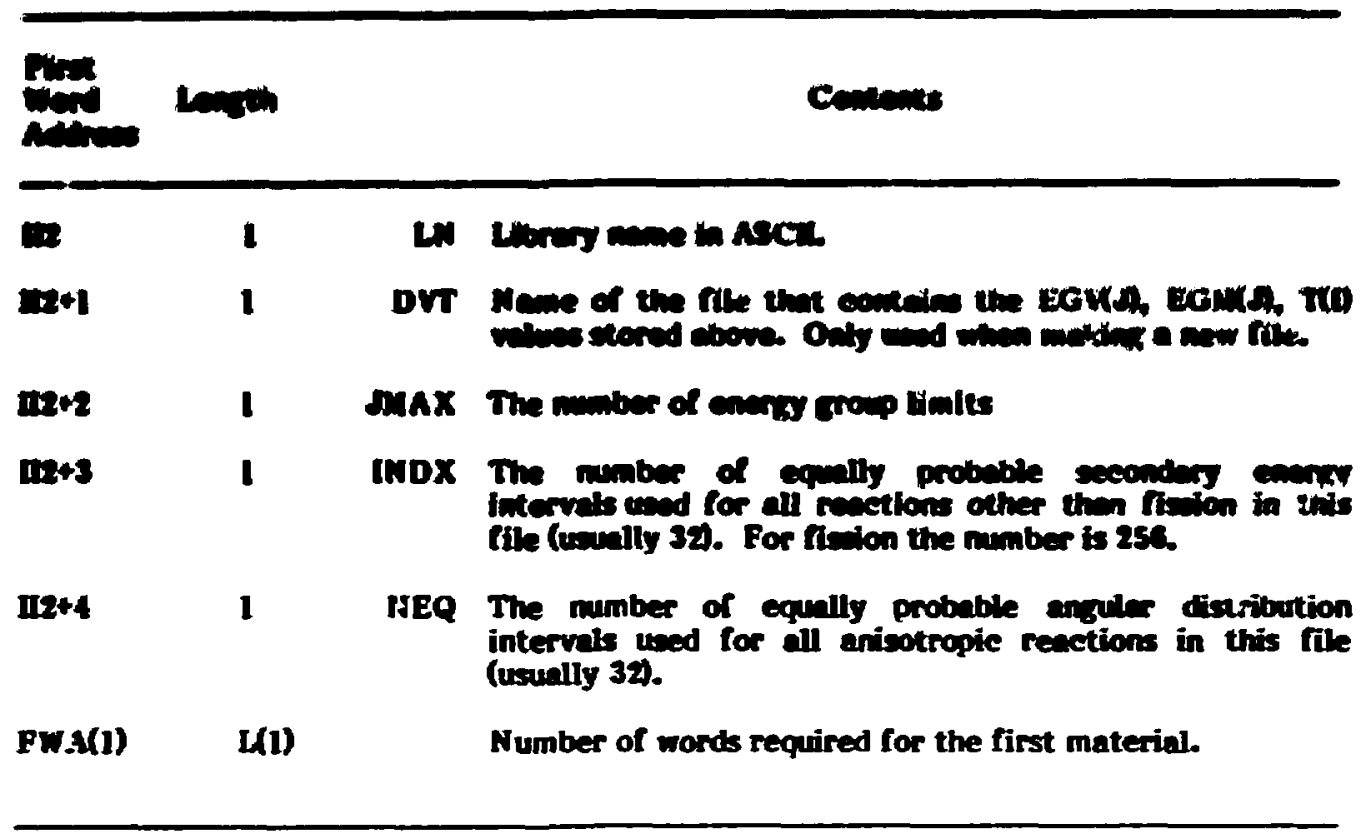

* The tables T(L), L-1,5 represent five subdivisions of the neutron speed (energy) regime such that muitiplying a neutron speed that lies within $T(L)$ by a value characteristic of the table [MU(L)] will give a value that has as its integer part the location within $T(L)$ that contains the index of the energy group in which the neutron belongs.

TABLE IX-A.1. Layout of the disk file for any material.
First word addresses
Contents

IWG(1)

IWG(2)

IW G(3)

AWG(4)
Number of words in control table.

The date of the last complete or partial evaluation in ASCII (yymmdd).

$$
\mathrm{ZA} \text { for the material }(1000 * \mathrm{Z}+\mathrm{A}) \text {. }
$$

Atomic number $\mathrm{Z}$ (floating point). Last six oetal digits is the FWA (IWW22) for the depletion-accretion tables (see Note 2).

$$
\text { IX }-6
$$




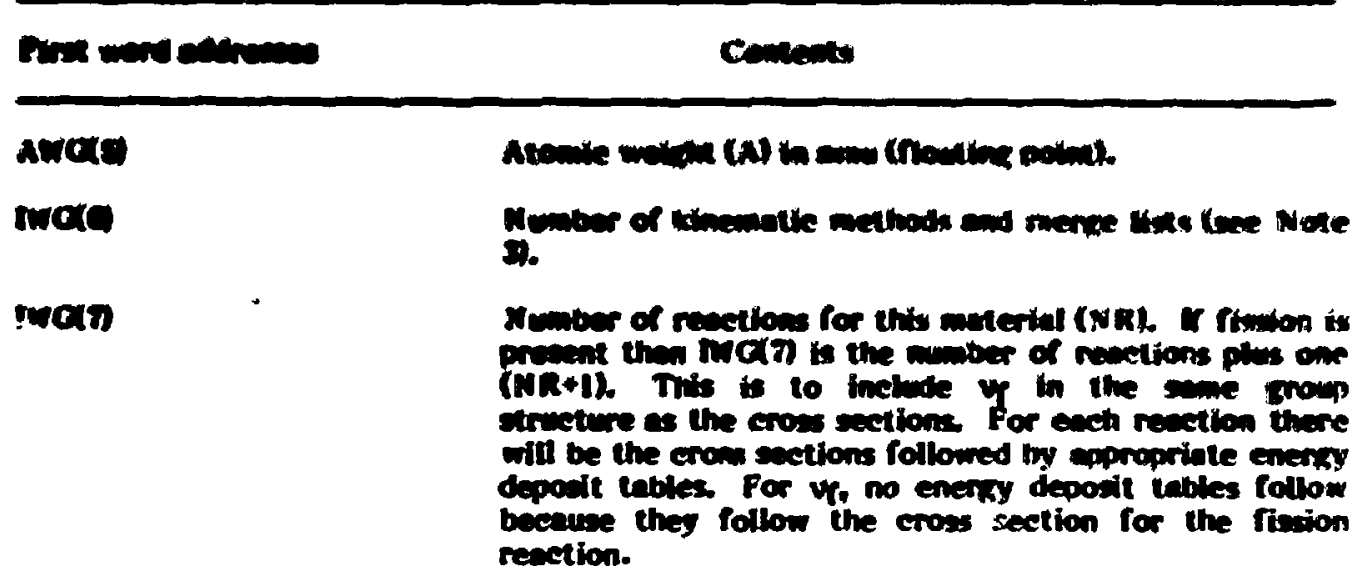

INGA

Number of wonds after last energy depusit list of the first reaction used to describe energy and/or angular distributions of seeondary neutrons for the speeifie reaction.

$\operatorname{IWC}(8+N-1)$

Number of words after energy deposit tables of the $\mathrm{N}^{\text {th }}$ reaction used to describe energy and/or angular distributions of secondary neutrons.

IWG(8+1WG(7)-1)

Number of words after reaction IWG( $\eta)$ used to describe energy and/or angular distributions of the secondary neutrons.

$I W W=8+I W G(\eta)$

IWG(IWW)

Kinematics method sentinel for the first reaction in the first merge list. This sentinel is used to specify the type of kinematies to be used in the collision routine.

$I W G(I W W+I W G(7)-1)$

Kinematics method sentinel for the last reaction in the first merge list.

IWG(IWW+IWG(7))

First merge number of first merge list. The reactions with the same merge number are added. This control is mainly used for neutron absorption reactions (see Note 3 ). 


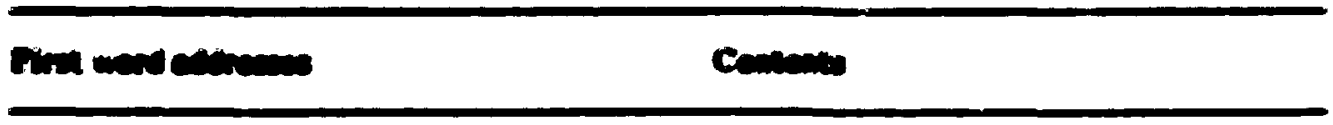

mortemeremert.

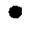

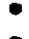

nounwezmoteman-1)

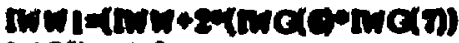
mothuld

[matnm1+1)

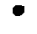

$-$

IWG(IWW1+24(IWG(n)-1)

$\operatorname{mw} 2=1 w 1+2 * \operatorname{mg}(7)$

AwG(IN)

IWG(IWW 2+I)

IพG(IWW 2+2)

IWG(IWW 2+3)

-

-

IWG(IWW 2+4*IWG(7)-1) 하하누

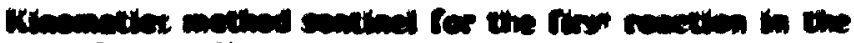

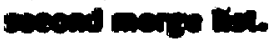

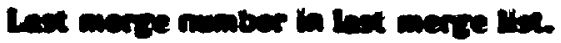

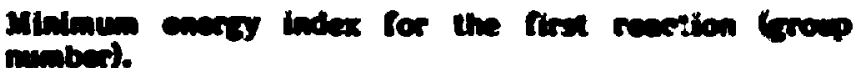
Maxinum enosy in lex (roop number) for the first reaction.

Maximum snergy index for the last resetion.

The reaction Q-value (MeV.

C (reaction type).

I (reaction property).

$\mathbf{S}$ (reaction modifier).
The reaction modifier $(S)$ for the last reaction. END HEADING AND CONTROL INFORMATION for an isotope.

Note: IWW 2 is recelculated after processing each reaction.

IWG(IWW 2+4*IWG(7))

The number of different types of energy deposit sets (controlled by data available in the evaluated library), for the first reaction. For example, one set can be all energy deposited except the energy of the emitted neutrons, or all energy deposited except the energy of the emitted neutrons and gamma rays, and/or others.

$I W W 3=[W W 2+4 *[W G(7)+]$

AWG(IWW 3)

First cross section for the first reaction.

IX -8 


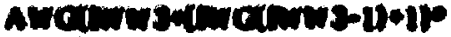

(omorimitit

ivarmin+|r-1]

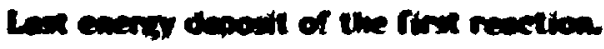

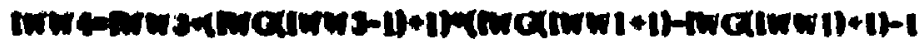

AwCinwatil

or

manwat1)
First edutional desertotor requined for the first

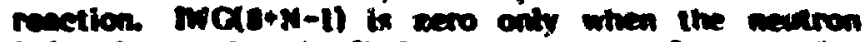
induced remetion ts fimion or copplure for reaction

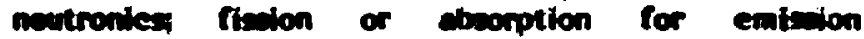
neutronies. The fiscion speetrum datn follow the $y$

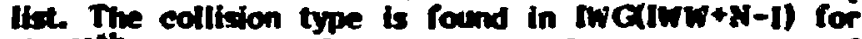
the Nth reaction. The meaning of the contents of AwG(IWW 4+1) or IWG(IWW +1$)$ is collision type dependent (see below).

The description of the data required for each collision type follows, these data are used by the subroutine GEN 2.

TABLE IX-A.2. Description of the data required for each collision type.

First word addresses

Contents

Collision Type 1 - Anisotropic Elastic Scattering in the COM System

IWG(IWW4+1)

Number of angular distributions specified for this reaction, i.e, the number of energies at which the angular iistributions are specified.

IW G(IWW 4+2)

Number of cosines used to define equally probable intervals in the integral for an anisotropic distribution (usually 33).

IW G(1WW4+3)

Energy group index for the least neutron energy at which an anisotropic distribution is given.

IWG(IWW 4+4)

Sentinel which describes whether the set of angular distributions are for a standard energy' mesh (each element has its angular distribution at the same energy) or a non-standard energy mesh (the angular distributions are only at the energies that are in the evaluated library). Standard $=0$, non-standard $=1$. All current data are in the non-standard mode.

$$
1 x-9
$$




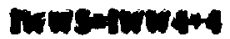

E...

Anctingstil

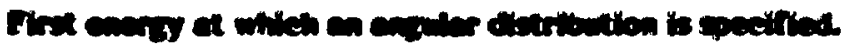

-

-

-

Ancinusuruc(nustin)

IWWe-iwWs+ing(In4+1) Awcimet1)

$\bullet$

$\bullet$

$\bullet$

AWG(IWW6+IWG(INW4+1)* IWG(IWW4+24)

Standard mesh

AWG(IWW5+1)

AWG(IWW5+IWG(IWW4+1)* IWG(IWW 4+2)
First word of the first angular distribution corresponding to the first energy of the standard energy mesh.

Last word of the last angular distribution corresponding to the last energy of the standard energy mesh.

Collision Type 2 - Absorption (i.e., no secondary neutrons from the reaction)

No additional information is needed.

Collision Type 3 - Level Seattering, Isotropic in the Center of Mass System AWG(IWW4+1) Energy of the excited level.

Collision Type 4 - Level Seattering Anisotropic in the Center of Mass System IW G(IWW 4+1) Number of angular distributions for this reaction.

IW G(IWW 4+2) Number of entries in an angular distribution (usually 33).

AWG(IWW 4+3) Energy of the excited level (MeV). 


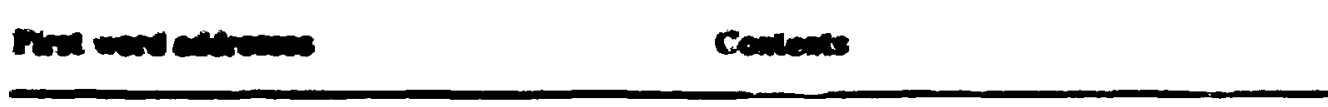

uncinguras

Ancherated

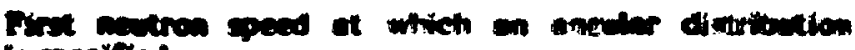

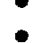

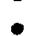

Awchwwstmoluwstin

nwwenwwstma(nw4+1)

AnG(nw6+1)

-

$-$

$\bullet$

AWG(TW6+1WG(1WW4+1)*

IWG(INW 4+2)) is speofried

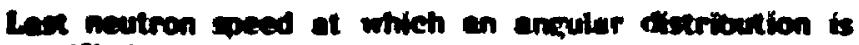
specilfed.

Pirst entry of the first angular distribution.

Collision Type 5, 7 - Temperature Model lsotropic in the Center of Mass System (Not used) IWG(IWW4+1) Number of temperatures for statistical model.

AWG(IWW4+2) Laboratory threshold for this reaction (in MeV).

AWG(IWW4+3) First temperature (in MeV).

-

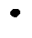

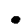

AWG(IWW4+2+IWG(IWW4+1)) Last temperature.

AWG(IWW4+3+IWG(IWW4+1))

$\bullet$

$\bullet$

First incident neutron energy at which the temperature model applies.

AWG(1WW4+2+2*IWG(IWW4+1)) Last incident neutron energy at which the temperature model applies.

Collision Type 6,8 - Combined Anisotropic Wide Level and Tabular Method

IWG(IWW 4+1)

Number of angular distributions for this reaction.

IWG(IWW4+2)

Number of entries in an angular distribution (usually 33).

AWG(1WW4+3)

Energy of the excited level ( $W$ ) (in MeV). 
Honsaniwats

Anconinst1)

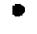

-

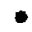

Awothws+h C(WW4+1)

Imwernins+no(iww4+1)

Awa(nw6+1)

-

-

$\bullet$

AWG(WW6+1WG(WW4+1)* IWG(ING4+2))

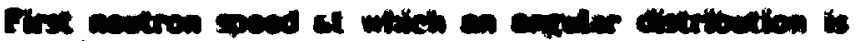
prectired.

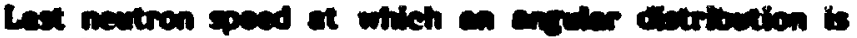
specifled.

First entry of the first angular distribution.

\section{IWW77=IWW6+IWG(ITH4+1)*(IWW4+2)+1}

IพG(Twพ7?)

IWG(IWW77+1)

AWG(IWW77+2)

AWG(IWW 77+3)

AWG(IWW77+4)

AWG(IWW77+IWG(IWW 77)+3)
Number of neutron energy groups for which the cross section is non-zero.

Energy index of first non-zero group cross section for wide level reaction.

Width of level in $\mathrm{MeV}(\mathrm{\Gamma})$.

$\operatorname{TAN}^{-1}\left(\frac{2}{\Gamma}(-|W|)\right)$, where $W=$ is the energy of the Ievel in MeV.

$\operatorname{TAN}^{-1}\left(\frac{2}{T}\left(\frac{A E(I W G(I W W 77+1))}{A+1}-|W|\right)\right)$,

where $A$ is the atomic mass in amu and $E$ is the lower bound of the least energy group for which the cross section is non-zero.

$\left.\operatorname{TAN}^{-1}\left(\frac{2}{\Gamma}\left(\frac{\mathrm{AE}(\text { IWG(IWW 77+IWG(IWW77)) }}{A+1}\right)-|W|\right)\right)$,

where $A$ is the atornic mass in amu, and $E$ is the upper bound of the greatest energy group for which the cross section is non-zero. 


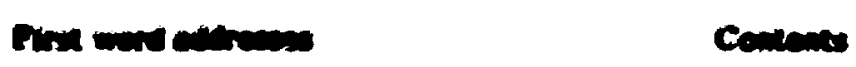

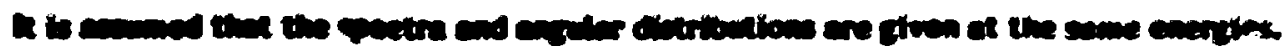

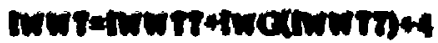

inctums+1)

Inctuw

Incinw7+9

MG(ITN7+4

AMG(INW7+5)

-

-

$A W G(1 W W 7+4+1 w G(1 w W 7+2))$

IWW8 $=[w W 7+4+1 W G(1 W W 7+2)$

AWG(IWW8+1)

$\bullet$

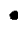

AWG(IWW 8+IWG(IWW 7+2)

*IWG(IWW 7+3))
Nunber of entirles in exest spectrum table of emitted nevinow (wantly 3x).

Aumber of speetre tebles, toen number of bisidemt merten encrgies at which a speetrum is given.

Number of entries in an anistropis distribution (trsunally 337.

Blank.

Least neutron speed at which a secondary neutron spectrum is given.

Greatest neutron speed at which a secondary neutron spectrum is given.

First entry in the first angular distribution table.

$I W W 9=I W W 8+I W G(I W W 7+2) * I W G(I W W 7+3)$

AWG(IWW 9+1)

-

-

$\bullet$

$A W G(1 W W 9+1 W G(I W W 7+1)$

*IWG(IWW7+2))
First neutron speed in the first secondary neutron emission spectrum.

Last neutron speed in the last secondary neutron emission spectrum. 


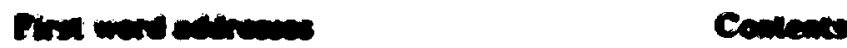

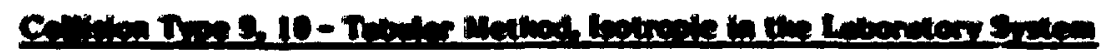
nothrwit)

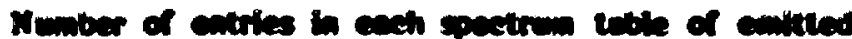

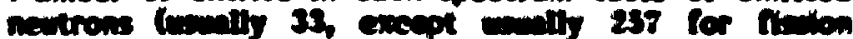
pection, soe Coltivion Type 19, 24.

Manwata

Awernistar

$\bullet$

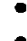

\section{AwG(Ww4+2+IwG(ww4+2))}

IWW5=IWW4+2+1WG(IWW4+2)

AwG(WW5+1)

-

-

$\cdot$

AWG(IWW5+IWG(IWW4+1)

*IWG(IWW4+2))

\section{Nuibuer of speetre tables.}

First neutron speed at which a spectrum is tiven.

Collision Type 11,12 - Cluster Model, Isotropic in the Center of Mass System

Level constant in center of mass system is the $Q$ of the reaction (see AWC(IWW2)).

Collision Type 13, 14, 15 - Tabular Method,

Energy and Angle Uncorrelated in Laboratory System

lt is assumed that the spectra and angular distributions are given at the same energies. IWG(IWW4+1)

Number of entries in each spectrum table of emitted neutrons (usually 33 ).

IWG(1WW4+2)

Number of spectra tables, i.e., the number of incident neutron energies at which a spectrum is given.

IWG(IWW 4+3)

Number of entries in an anistropic distribution (usually 33).

IWG(IWW 4+4)

Blank.

AWG(IWW 4+5)

First neutron speed at which a spectrum is given.

$$
\text { IX }-14
$$




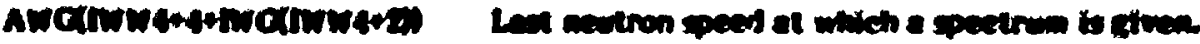

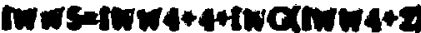

\section{AwC(nWw+1)}

-

-

-

\section{AWG(IWWs+IWG(WW4+2)}

*nG(n)4+3)
First entry in the rirst angular distribution table.

IMW6=IWW5+IWG(WW4+2)*IWG(IWW4+3)

AWG(IWW6+1)

First neutron speed in the first emission spectrum.

.

$-$

AWG(IWWG+IWG(IWW4+I)

*IWG(IWW4+2))

Last neutron speed in the last emission spectrum.

Collision Type 16, 17, - Combined Anistropic Tabular and Level Method

IWG(IWW4+1)

IWG(IWW 4+2)

$A W G(1 W W 4+3)$

IWW $5=I W W 4+3$

AWG(1WW5+1)

$\bullet$

-

-

AWG(IWW5+IW G(IWW4+1))

IWW 6=IWW5+IWG $($ IWW 4+1)
Number of angular distributions for this reaction.

Number of entries in an angular distribution (usually 33).

Energy of excited level.

First neutron speed at which an angular distribution is specified.

Last neutron speed at which an angular distribution is specified.

First entry of first angular distribution. 
Awo(n)wermanmat1)

anclimenter

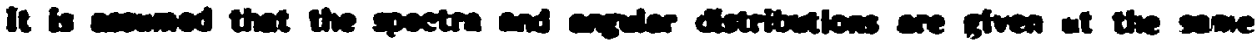
concine

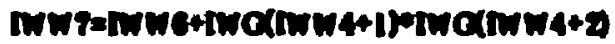

iwa(nw7+1)

IWG(WWr+2)

IWG(IwW7+3)

IWG(IWW7+4)

AWG(IWW7+5)

-

$-$

AWG(IWW7+4+IWG(IWW7+2))

IWW $8=1 W W 7+4+i W G(1 W W 7+2)$

AWG(WW 8+1)

-

-

AWG(IWW 8+IWG(IWW 7+2)

*IWG(IWW7+3))
Number of entries in anch spectrun teble of enitted neutione (wovilly 33.

Number of spectra tabies, i.en the number of incident neutron energies at which the speetrum is given.

Number of entries in an anistropic distribution (usually 33).

Blank.

First neutron speed at which a spectrum is given.

Last neutron speed at which a spectrum is given.

First entry in the first angular distribution table.

Last entry in the last angular distribution table.

IWW 9=IWW 8+IWG(IWW 7+2)*IW G(IWW7+3)

AWG(IWW9+1)

$-$

-

AWG(IWW 9+IWG(IWW 7+1)

*IWG(IWW 7+2))
First neutron speed in the first emission spectrum.

Last neutron speed in the last emission spectrum. 


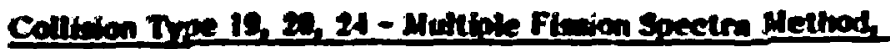

\section{Isotropic in Leborntory Syatern}

Both $q$ and $y$ are given as a functon of exengyi of is treated as ary other resetion eross seetion, it has multiple spectra using models 9, 10, following it (sce the description of models 9 and 10.

\section{Collimion Troe 21, 22,23 - Fision Spectra Method,} Eotropic in Laboratory System (Not used)

of is treated as any other reaction, vi has no energy deposits and only one spectrum (energy independent).

\section{IWG(IWW +1)}

AFG(IWW4+2)

-

-

AWG(IWW4+IWG(IWW4+1))
Number of entries in fission spectrum.

First neutron speed in fission speetrum.
IAD(IWW 22)

IAD(IWW 22+1)

IAD(IWW 22+2)

IAD (IWW 22+3)

IAD(IWW 22+4)

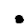

Accretion/Depletion Data for the Material

Last neutron speed in the fission spectrum.

Same as $9,10$.

Number of aceretion/depletion lists to follow.

Number of reactions considered in the first list.

$\mathrm{C}$-value for the first rexction of the first list.

Number of products from the first reaction of the first list.

First product from the first reaction of the first list.

END OF DATA FOR THE MATERIAL

\section{GAMMA RAY PRODUCTION DISK FILE}

The layout of the gamma ray production data is an exact duplicate of the neutron file. The data required for the calculation of the photon production line and spectra follows. 


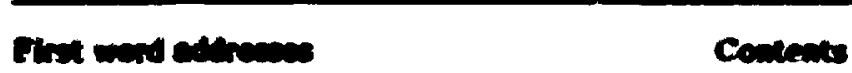

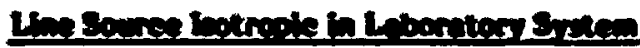

Awc(n)w4+1)

Bnesy of the the (Men.

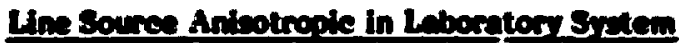

[wa(ww4+1)

Iwetiwwety

AnG(IWW4+3)

IwW $5=1 \% W 4+3$

AWG(IWW5+1)

-

-

AWG(IWW5+IWG(IWW4+1))

IWW6=IWW 5+IWG(IWW4+1)

A WG(IWW6+1)

-

-

AWG(IWW 6+IWG(IWW4+1)

*IW G(IWW4+2))

\section{Tabular Spectra Isotropic in Laboratory System}

IWG(1WW4+1)

IWG(IWW4+2)

AWG(IWW4+3)

-

$\bullet$

AWG(IWW 4+2+IWG(IWW4+2))

Last neutron speed at which a spectrum is given.
Last neutron speed at which an angular distribution is specified.

First entry in the first photon angular distribution.
Last entry in the last photon angular distribution.

Number of entries in each spectrum table of emitted photons (usually 33 ).

The number of neutron speeds at which spectra are given.

First neutron speed at which a spectrum is given.

$I W W 5=I W W 4+2+I W G(I W W 4+2)$

IX -18 


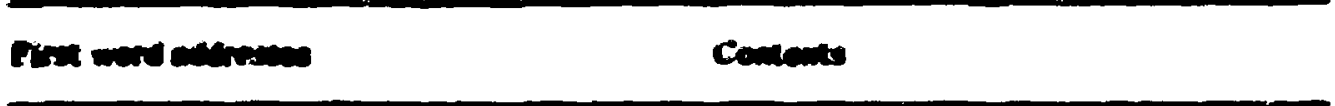

Ancturns+1)

First ands) in the firse spectrum.

(

-

Ancumbenchentint)

anctinnt+2)

Last energy in the last geetrum.

Tbuler Speetre - Anieptragic in Leboratory Syctem

ING(1)

ING(INW4+2)

IGG(IwW4+3)

IWG(IWWA+4)

AWG(IWW4+5)

-

AWG(IWW1+4+IWG(IWW4+2))

IWW $5=$ IWW 4+4+IWG(IWW4+2)

AWG(IWW5+1)

AWG(IWW5+IWG(IWW4+2)

*IWG(IWW4+3))
Number of enengies in each speetrum tabie of emitted photons (unually 33).

Number of spectra tables, i.e., the number of incident neutron energies at which the spectra are given.

Number of entries in an anisotropic angular distribution (usually 33).

Blank.

First neutron speed at which a photon spectrum is given.

Last neutron sperc at which a photon speetrum is given.

First entry in the first table of photon angular distributions.

$\operatorname{IWW6} 6=1 W W 5+I W G(I W W 4+2) * I W G(1 W W 4+3)$

AWG(IWW6+1)

First photon energy in the first photon spectrum.

Last entry in the last table of photon angular distributions.

AWG(IWW6+1WG(IWW4+1)

*IWG(IWW4+2))

Last photon energy in the last photon spectrum.

$$
\text { IX }-19
$$


thing

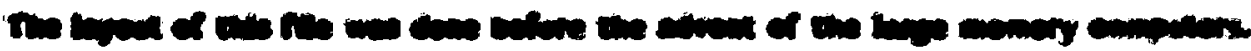

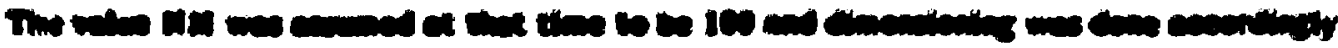

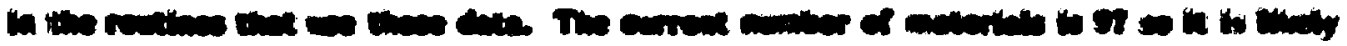

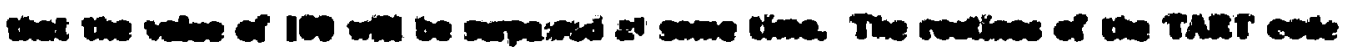
מוs

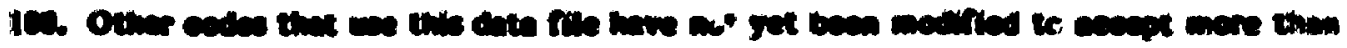

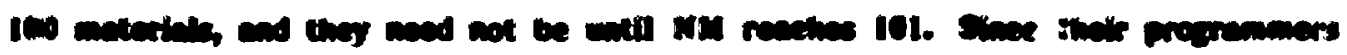

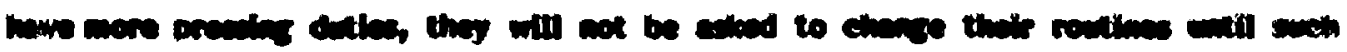

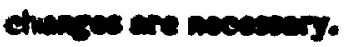

\section{Note 23}

One of the eodes that une this date file has need for depletion/ecuretion date. Thisy are stoced at the end of the data for each material. Since this is a specul use that will not interfere with other uses of the file and since the $Z$ value cannot exeeed three deeimal dits, the addres of the depletion/eccretion data is stored in the last six cetal digits of this word. This is the only case of packing a word in the file.

\section{Note 3:}

Frequent reference is made to kinematics methods, merge lists, and collision types. Kinematics methods and collision types are synonymous in the sense that a particular type of collision implies tine use of a kinematic method for determining the energy and angular distributions of secondary particles (i.e., neutrons or photons). Merge lists are lists of the reactions for the material that indicates whether ur not certain reactions are to be combined depending on the mode in which the code that uses the data is to be run. There are two modes, reaction neutronies and emission neutronics. In the former, each nuclear reaction is dealt with separatel: so no combination of reactions is done. For emission neutronies, all neutron producing reactions are dealt with individually, but all reactions that do not produce neutrons are combined into a single absorption reaction. In thie terminology of the ENDL encoding system, all reactions with $\mathrm{C} \leq 31$ are treated individually and all reactions with $32 \leq \mathrm{C} \leq 49$ are lumped together. For definitions of the "C" values for the reactions, see UCRL 50400, Vol. 4, Rev. 1, pp. 8 and 9. The lumping together of the absorption reactions is done by the subroutine that prepares the data from this file for use by the code that will be using the data (i.e., the combining of reactions, if any, takes place at generation time for the problem to be run). 


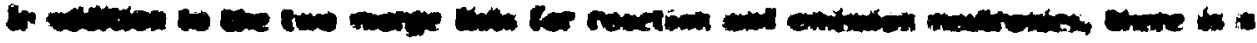

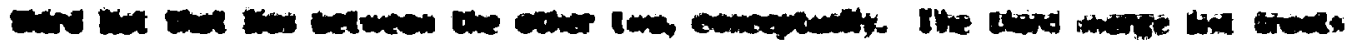

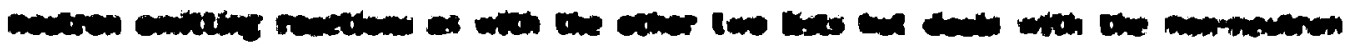

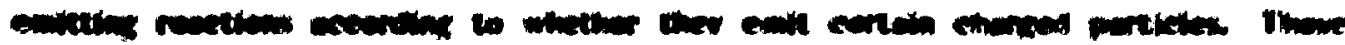

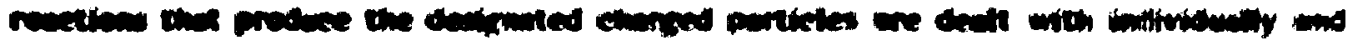

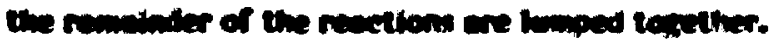

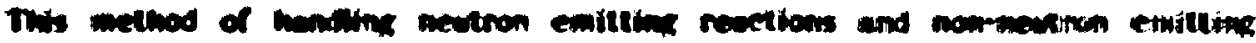

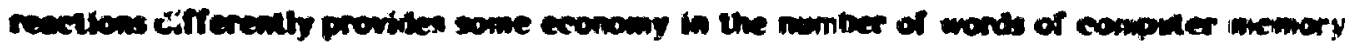

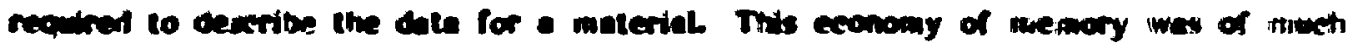
creater importance a decade 40 then it is now because of the much simulker inemory expacity of componters that existed at that time. Given the curvent unerwory size of the computers on which codes that we this fike ren, it is likely that if the file were to be designed now, no distinetion would be made between neutron producing and non-neutron producing reactions and all problems would be tun in the reaction neutronies mode. To revise the codes that use this file, such that they would run only in the reaction neutronies mode, would not be cost-effective.

$$
1 x-21 / 1 x-22
$$


chaptea $x$ ucPolv T

$$
x-1 / x-2
$$




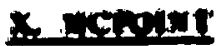

\section{A. unowotem

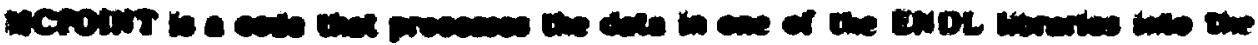

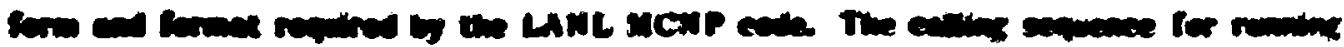 incounters}

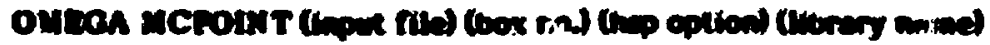

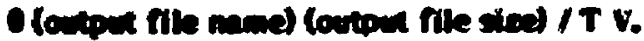

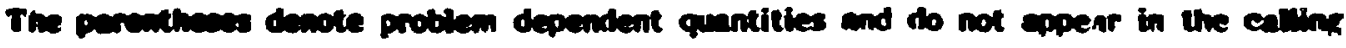

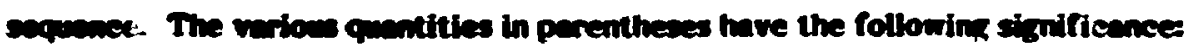

inpert file

bou no.

hip option

library name

0

output file name

output file size the fle required by MCPOINT for input

user's box number

MCPOINT produces output that is descriptive and/or diegnostic for the problem. This ontput may be directed to the FR80 (use "fr80" for option), the high speed printer (use "hsp" for option), or kept for inspection (use "debug" for option) and/or output to RJET or other medium using ALLOUT.

the ENDL library that is being used to produce the file for the MCN P code.

The zero in this position indicates that the library exists as a file. The zero musi be included in the calling sequence.

the name of the file to be created as a data file for use by the MCN P code.

the size of the output file to be created in units of 100000 words. In the example above, the output file will be created as 2000000 words. When the code is finished it will shrink the file to the size needed.

An example of running MCPOINT using an input file MCIN with a library called ND821201 to make a data file MCN POUT that can be used with the MCN P code is: omega mepoint mein $t 64$ fr80 nd8212010 menpout $20 / .9 .999$ 


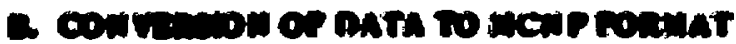

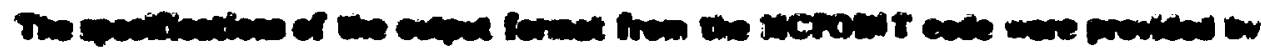

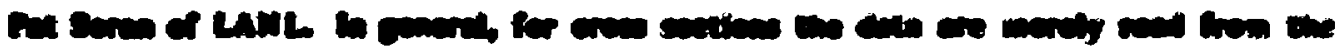

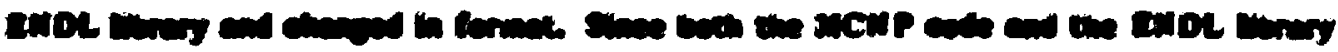

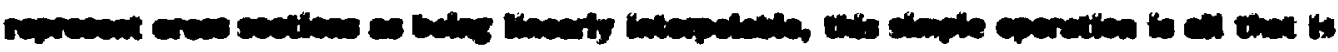

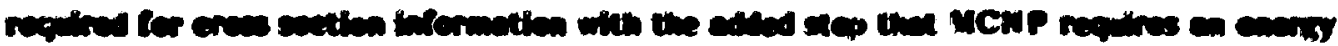

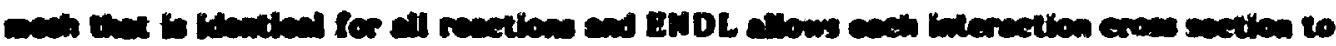

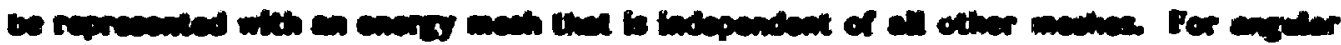

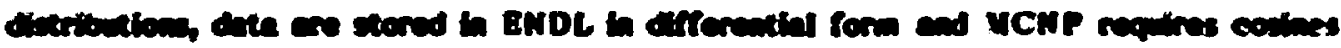

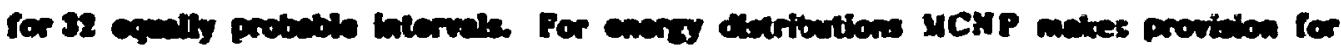

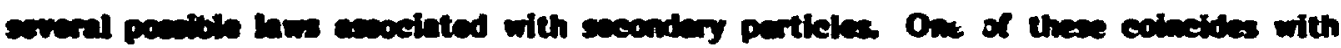
the method und in ENDL, namely, all secondary enersy distributions in ENDL are diven explicithy at a mech of incident energies such that linear interpolation betweer wecessive entries (on a unit base basis) will yield a distribution for an intermediate ensory. The conversion that is required is to ehange from the differential form used by ENDL to 32 equally probable entries of secondary energies used by MCN P. 